\title{
One-Pot Transformation of Furans into 1-Azaspirocyclic Alkaloid Frameworks Induced by Visible Light
}

AUTHORS: Stela Hoxha, Dimitris Kalaitzakis, Artemis Bosveli, Tamsyn

Montagnon and Georgios Vassilikogiannakis*

Department of Chemistry, University of Crete

VasilikaVouton, 71003, Iraklion, Crete, Greece

E-mail: vasil@uoc.gr

\section{$\underline{\text { Table of contents }}$}

Part A: General methods, experimental procedures

$\mathrm{S} 1-\mathrm{S} 19$

Part B: Copies of ${ }^{1} \mathrm{H}-\mathrm{NMR},{ }^{13} \mathrm{C}-\mathrm{NMR}, \mathrm{COSY}, \mathrm{HSQC}, \mathrm{HMBC}$ and NOE Spectra. $\mathrm{S} 20-\mathrm{S} 70$ 


\section{Part A: General methods, experimental procedures}

\section{General methods}

NMR data were obtained for ${ }^{1} \mathrm{H}$ at $500 \mathrm{MHz}$ and for ${ }^{13} \mathrm{C}$ at $125 \mathrm{MHz}$. HRMS data were recorded on a Q-Exactive Plus Orbitrap MS, using an ESI ionization source.

\section{Synthesis of substrates of type 1 and 2}

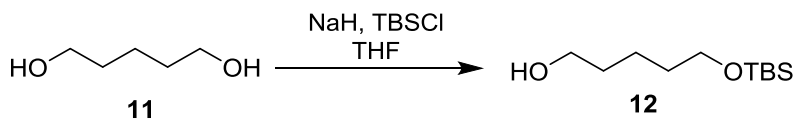

\section{5-((tert-butyldimethylsilyl)oxy)pentan-1-ol (12)}

To a solution of diol $11(1.05 \mathrm{~mL}, 10 \mathrm{mmol})$ in anhydrous THF (50 mL), under an argon atmosphere and at $0{ }^{\circ} \mathrm{C}$, NaHMDS (12 mL, $1 \mathrm{M}$ in THF, $12 \mathrm{mmol}$,) was added dropwise and the solution stirred at the same temperature for $1 \mathrm{~h}$. TBSCl (1.96 g, 13 mmol) in anhydrous THF (5 mL) was added in one portion with the reaction still maintained at $0{ }^{\circ} \mathrm{C}$. The solution was left to reach $\mathrm{rt}$ and then stirred for a further $18 \mathrm{~h}$. After completion of the reaction, as indicated by tlc analysis, a saturated aqueous solution of $\mathrm{NH}_{4} \mathrm{Cl}(40 \mathrm{~mL})$ was added and the mixture was extracted with $\mathrm{Et}_{2} \mathrm{O}(2 \times$ $40 \mathrm{~mL}$ ). The combined organic layers were dried over $\mathrm{MgSO}_{4}$, filtered and concentrated in vacuo. The residue was purified by flash column chromatography ( silica gel, petroleum ether : EtOAc $=20: 1 \rightarrow 10: 1$ ) to afford 12 as a colorless oil. Yield $1.308 \mathrm{~g}(60 \%)$.

${ }^{1} \mathrm{H}$ NMR (500 MHz, $\left.\mathrm{CDCl}_{3}\right): 3.65$ (m, 2H), 3.62 (t, J=6.5 Hz, 2H), 1.57 (m, 5H), 1.41 $(\mathrm{m}, 2 \mathrm{H}), 0.89$ (s, 9H), 0.05 (s, 6H) ppm; $\left.{ }^{13} \mathrm{C} \mathrm{NMR} \mathrm{(125} \mathrm{MHz,} \mathrm{CDCl}_{3}\right)$ : 63.1, 62.9, 32.5 (2C), 25.9 (3C), 22.0, 18.3, -5.3 (2C) ppm.

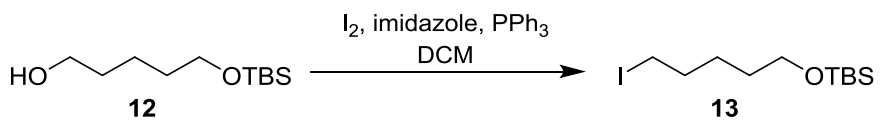

\section{tert-butyl((5-iodopentyl)oxy)dimethylsilane $(\mathbf{1 3})^{2}$}

To a solution of alcohol $12(1.308 \mathrm{~g}, 6.0 \mathrm{mmol})$ in anhydrous $\mathrm{CH}_{2} \mathrm{Cl}_{2}(15 \mathrm{~mL})$, under argon atmosphere at $0{ }^{\circ} \mathrm{C}, \mathrm{PPh}_{3}(1.89 \mathrm{~g}, 7.2 \mathrm{mmol})$ and $\mathrm{I}_{2}(1.83 \mathrm{~g}, 7.2 \mathrm{mmol})$ were added. The solution was stirred for $5 \mathrm{~min}$. Afterwards, imidazole (1.02 g, $15 \mathrm{mmol})$ was added at the same temperature. The resulting solution was warmed to room temperature and stirred for a further $1.5 \mathrm{~h}$. After completion of the reaction, as indicated by tlc analysis, a saturated aqueous solution of $\mathrm{Na}_{2} \mathrm{~S}_{2} \mathrm{O}_{3}(10 \mathrm{~mL})$ was added and the solution stirred for $30 \mathrm{~min}$. The layers were separated and the organic phase was dried over $\mathrm{Na}_{2} \mathrm{SO}_{4}$, filtered and concentrated in vacuo. The residue was purified by flash column chromatography (silica gel, petroleum ether) to afford the iodide $\mathbf{1 3}$ as a slightly yellow oil. Yield $1.712 \mathrm{~g}(87 \%)$.

\footnotetext{
${ }^{1}$ Riefert, A.; Maier, M. E. Synthesis 2018, 50, 3131-3145.

${ }^{2}$ Ellwood, A. R.; Porter, M. J. J. Org. Chem. 2009, 74, 7982-7985.
} 
${ }^{1} \mathrm{H}$ NMR (500 MHz, $\left.\mathrm{CDCl}_{3}\right): 3.61(\mathrm{t}, J=6.3 \mathrm{~Hz}, 2 \mathrm{H}), 3.19(\mathrm{t}, J=7.0 \mathrm{~Hz}, 2 \mathrm{H}), 1.84$ (quint, $J=7.2 \mathrm{~Hz}, 2 \mathrm{H}), 1.53(\mathrm{~m}, 2 \mathrm{H}), 1.44(\mathrm{~m}, 2 \mathrm{H}), 0.89(\mathrm{~s}, 9 \mathrm{H}), 0.05(\mathrm{~s}, 6 \mathrm{H}) \mathrm{ppm} ;{ }^{13} \mathrm{C}$ NMR (125 MHz, $\mathrm{CDCl}_{3}$ ): 62.8, 33.3, 31.7, 26.9, 25.9 (3C), 18.3, 7.0, -5.3 (2C) ppm.

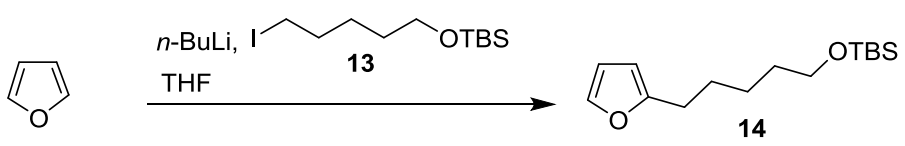

\section{tert-butyl((5-(furan-2-yl)pentyl)oxy)dimethylsilane (14) ${ }^{3}$}

To a solution of furan $(1.14 \mathrm{~mL}, 15.7 \mathrm{mmol})$ in anhydrous THF $(15 \mathrm{~mL})$, under an argon atmosphere and at $0{ }^{\circ} \mathrm{C}$, was added dropwise a solution of $n$-BuLi $(8.16 \mathrm{~mL}, 1.6$ $\mathrm{M}$ in hexane, $13 \mathrm{mmol}$ ). The solution was stirred for a further $30 \mathrm{~min}$ at the same temperature. Afterwards, a solution of the iodide $\mathbf{1 3}(1.71 \mathrm{~g}, 5.22 \mathrm{mmol})$ in anhydrous THF ( $5 \mathrm{~mL}$ ) was added slowly. The solution was then warmed to room temperature and stirred for $1 \mathrm{~h}$. After completion of the reaction, as indicated by tlc analysis, the reaction was quenched with a saturated aqueous solution of $\mathrm{NH}_{4} \mathrm{Cl}(10 \mathrm{~mL})$ and the resulting mixture was extracted with $\mathrm{Et}_{2} \mathrm{O}(20 \mathrm{~mL})$. The organic layer was separated, dried over $\mathrm{Na}_{2} \mathrm{SO}_{4}$ and concentrated in vacuo. The product was purified by flash column chromatography (silica gel, petroleum ether) to afford $\mathbf{1 4}$ as a slightly yellow oil. Yield $811 \mathrm{mg}(58 \%)$.

${ }^{1} \mathrm{H}$ NMR (500 MHz, $\mathrm{CDCl}_{3}$ ): $7.29(\mathrm{~m}, 1 \mathrm{H}), 6.27$ (dd, $\left.J_{l}=3.0 \mathrm{~Hz}, J_{2}=2.0 \mathrm{~Hz}, 1 \mathrm{H}\right), 5.97$ $\left(\mathrm{dd}, J_{l}=3.0 \mathrm{~Hz}, J_{2}=0.7 \mathrm{~Hz}, 1 \mathrm{H}\right), 3.60(\mathrm{t}, J=6.6 \mathrm{~Hz}, 2 \mathrm{H}), 2.62(\mathrm{t}, J=7.6 \mathrm{~Hz}, 2 \mathrm{H}), 1.65$ (quint, $J=7.5 \mathrm{~Hz}, 2 \mathrm{H}), 1.54(\mathrm{~m}, 2 \mathrm{H}), 1.38(\mathrm{~m}, 2 \mathrm{H}), 0.89(\mathrm{~s}, 9 \mathrm{H}), 0.04(\mathrm{~s}, 6 \mathrm{H}) \mathrm{ppm} ;{ }^{13} \mathrm{C}$ NMR (125 MHz, $\left.\mathrm{CDCl}_{3}\right)$ : 156.4, 140.6, 110.0, 104.6, 63.1, 32.6, 27.9, 27.8, 26.0 (3C), 25.4, 18.3, -5.3 (2C) ppm.

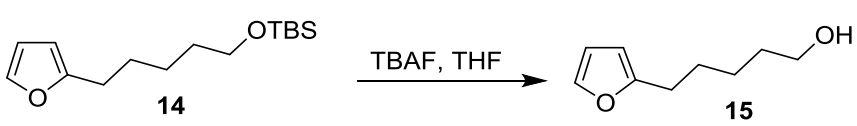

\section{5-(furan-2-yl)pentan-1-ol (15) ${ }^{4}$}

To a solution of furan $\mathbf{1 4}(811 \mathrm{mg}, 3.03 \mathrm{mmol})$ in THF $(5 \mathrm{~mL})$ at $\mathrm{rt}$, TBAF $(6.06 \mathrm{~mL}$, $6.06 \mathrm{mmol}, 1 \mathrm{M}$ in THF) was added and the reaction solution was stirred for $2 \mathrm{~h}$ at the same temperature. After the completion of the reaction, as indicated by tlc analysis ( 2 h), $\mathrm{Et}_{2} \mathrm{O}(15 \mathrm{~mL})$ was added and the organic phase was washed with distilled water $(10 \mathrm{~mL})$ and then with brine $(10 \mathrm{~mL})$. The organic phase was dried over $\mathrm{Na}_{2} \mathrm{SO}_{4}$, filtered and concentrated in vacuo. The residue was purified by flash column chromatography (silica gel, petroleum ether : ethyl acetate $=4: 1$ ) to afford $\mathbf{1 5}$ as a slightly yellow oil. Yield $457 \mathrm{mg}(98 \%)$.

${ }^{1} \mathrm{H}$ NMR (500 MHz, $\mathrm{CDCl}_{3}$ ): $7.29\left(\mathrm{dd}, J_{l}=1.8 \mathrm{~Hz}, J_{2}=0.8 \mathrm{~Hz}, 1 \mathrm{H}\right), 6.27$ (dd, $J_{l}=3.1$ $\left.\mathrm{Hz}, J_{2}=1.9 \mathrm{~Hz}, 1 \mathrm{H}\right), 5.98\left(\mathrm{dd}, J_{l}=3.1 \mathrm{~Hz}, J_{2}=0.8 \mathrm{~Hz}, 1 \mathrm{H}\right), 3.65(\mathrm{t}, J=6.5 \mathrm{~Hz}, 2 \mathrm{H}), 2.64$ (t, $J=7.5 \mathrm{~Hz}, 2 \mathrm{H}), 1.68$ (quint, $J=7.7 \mathrm{~Hz}, 2 \mathrm{H}), 1.60(\mathrm{~m}, 2 \mathrm{H}), 1.42(\mathrm{~m}, 2 \mathrm{H}) \mathrm{ppm} ;{ }^{13} \mathrm{C}$ NMR (125 MHz, $\left.\mathrm{CDCl}_{3}\right): 156.2,140.7,110.0,104.7,62.9,32.5,27.9,27.8,25.3$ ppm.

\footnotetext{
${ }^{3}$ Allegretti, P. A.; Ferreira, E. M. Org. Lett. 2011, 13, 5924-5927.

${ }^{4}$ Cesati, R. R.; Dwyer, G.; Jones, R. C.; Hayes, M. P.; Yalamanchili, P.; Casebier, D. S. Org. Lett. 2007, 9, 5617-5620.
} 


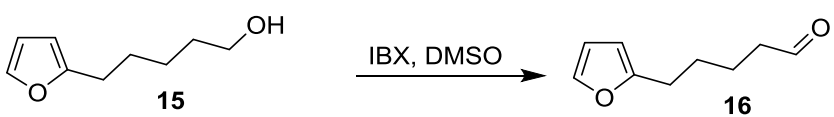

\section{5-(furan-2-yl)pentanal (16) ${ }^{4}$}

2-Iodoxybenzoic acid (1.66 g, $5.94 \mathrm{mmol})$ in dry DMSO $(9 \mathrm{~mL})$ at $\mathrm{rt}$, was stirred until the mixture became transparent. A solution of the alcohol 15 (457 mg, $2.97 \mathrm{mmol})$ in dry DMSO $(1 \mathrm{~mL})$ was added dropwise and the reaction was stirred at the same temperature. After completion of the reaction, as indicated by tlc analysis $(2 \mathrm{~h})$, $\mathrm{CH}_{2} \mathrm{Cl}_{2}$ (15 mL) was added. The mixture was cooled to $0{ }^{\circ} \mathrm{C}$ using an ice bath and distilled water $(10 \mathrm{~mL})$ was added. After precipitation of solids, the mixture was filtered through cotton wool and the layers of the resulting biphasic solution were separated. The organic phase was washed with a saturated aqueous solution of $\mathrm{NaHCO}_{3}(3 \times 10 \mathrm{~mL})$ and then with brine $(10 \mathrm{~mL})$. The organic phase was dried over $\mathrm{Na}_{2} \mathrm{SO}_{4}$, filtered and concentrated in vacuo. The residue was purified by flash column chromatography (silica gel, petroleum ether : EtOAc $=60: 1$ ) to afford $\mathbf{1 6}$ as a colorless oil. Yield $352 \mathrm{mg}(78 \%)$.

${ }^{1} \mathrm{H}$ NMR (500 MHz, $\left.\mathrm{CDCl}_{3}\right): 9.76(\mathrm{t}, J=1.7 \mathrm{~Hz}, 1 \mathrm{H}), 7.29\left(\mathrm{dd}, J_{l}=1.8 \mathrm{~Hz}, J_{2}=0.8 \mathrm{~Hz}\right.$, $1 \mathrm{H}), 6.28\left(\mathrm{dd}, J_{l}=3.1 \mathrm{~Hz}, J_{2}=1.8 \mathrm{~Hz}, 1 \mathrm{H}\right), 5.99\left(\mathrm{dd}, J_{l}=3.1 \mathrm{~Hz}, J_{2}=0.8 \mathrm{~Hz}, 1 \mathrm{H}\right), 2.65$ $(\mathrm{m}, 2 \mathrm{H}), 2.46(\mathrm{~m}, 2 \mathrm{H}), 1.69(\mathrm{~m}, 4 \mathrm{H}) \mathrm{ppm} ;{ }^{13} \mathrm{C} \mathrm{NMR}\left(125 \mathrm{MHz}, \mathrm{CDCl}_{3}\right)$ : 202.4, 155.6, $140.9,110.1,105.0,43.6,27.7,27.5,21.5 \mathrm{ppm}$.

General experimental procedure for the synthesis of compounds 18, 19, 1b, 1c, $2 \mathrm{~b}$ and $2 \mathrm{c}$.

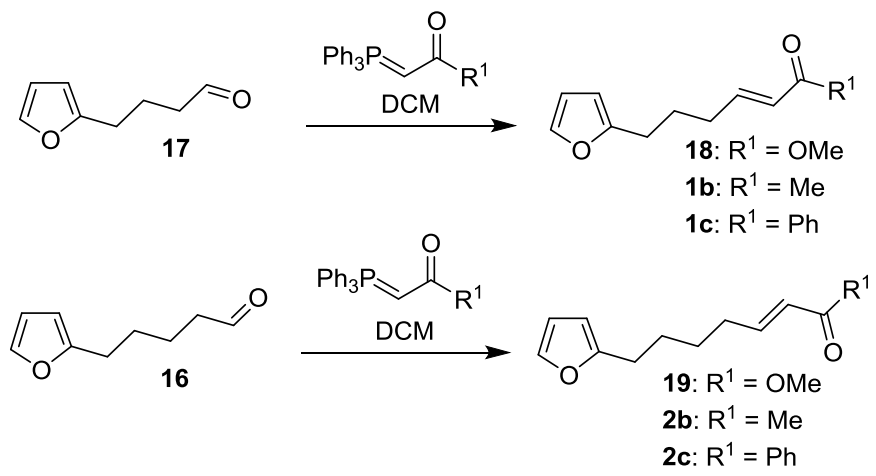

To a solution of the corresponding aldehyde (for $17 ;^{5} 690 \mathrm{mg}, 5 \mathrm{mmol}$ towards $\mathbf{1 8}$, $138 \mathrm{mg}, 1 \mathrm{mmol}$ towards $\mathbf{1 b}$ and $\mathbf{1 c}$; for $\mathbf{1 6} ; 274 \mathrm{mg}, 1.8 \mathrm{mmol}$ towards $\mathbf{1 9}$ and $\mathbf{2 c}, 78$ $\mathrm{mg}, 0.51 \mathrm{mmol}$ towards $\mathbf{2 b}$ ) in DCM (10 mL towards $\mathbf{1 8}, 2 \mathrm{~mL}$ towards $\mathbf{1 b}$ and $\mathbf{1 c}, 4$ $\mathrm{mL}$ towards $\mathbf{1 9}$ and $\mathbf{2 c}$ and $1 \mathrm{~mL}$ towards $\mathbf{2 b}$ ) at room temperature, the corresponding triphenyl phosphonium ylide (for methyl 2-(triphenylphosphoranylidene)acetate, 3.34 $\mathrm{g}, \quad 10 \mathrm{mmol}$ towards $\mathbf{1 8}, 1.25 \mathrm{~g}, 3.6 \mathrm{mmol}$ towards $\mathbf{1 9}$; or for 1 (triphenylphosphoranylidene)-2-propanone $637 \mathrm{mg}, 2 \mathrm{mmol}$ towards $\mathbf{1 b}$ and $325 \mathrm{mg}$, $1.02 \mathrm{mmol}$ towards $\mathbf{2 b}$; or for 1-phenyl-2-(triphenylphosphoranylidene)-ethanone, $761 \mathrm{mg}, 2 \mathrm{mmol}$ towards $\mathbf{1 c}$ and $1.71 \mathrm{~g}, 4.5 \mathrm{mmol}$ towards $2 \mathrm{c}$ ) was added in one portion. The resulting solution was stirred at the same temperature. After complete

\footnotetext{
${ }^{5}$ Kalaitzakis, D.; Montagnon, T.; Antonatou, E.; Vassilikogiannakis, G. Org. Lett. 2013, 15, 37143717.
} 
consumption of the starting material was indicated by tlc analysis $(48 \mathrm{~h})$, the solvent was removed in vacuo. The crude residue was purified by flash column chromatography (silica gel, petroleum ether : EtOAc).

\section{(E)-7-(furan-2-yl)hept-3-en-2-one (1b) ${ }^{6}$}

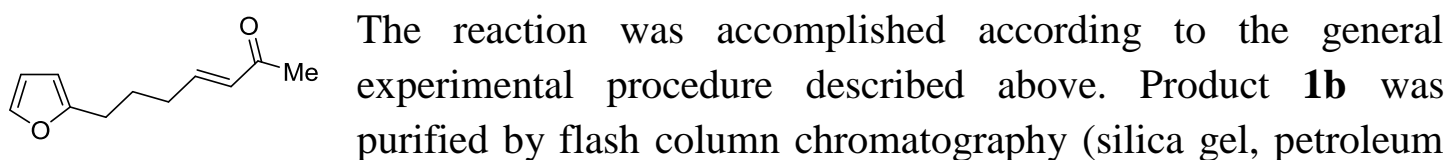
ether : EtOAc $=70: 1)$. Yield $140.6 \mathrm{mg}(79 \%)$ of a yellow oil. ${ }^{1} \mathrm{H}$ NMR $(500 \mathrm{MHz}$, $\left.\mathrm{CDCl}_{3}\right): 7.30(\mathrm{~s}, 1 \mathrm{H}), 6.78\left(\mathrm{dt}, J_{l}=15.9 \mathrm{~Hz}, J_{2}=6.8 \mathrm{~Hz}, 1 \mathrm{H}\right), 6.28(\mathrm{~s}, 1 \mathrm{H}), 6.08(\mathrm{~d}$, $J=15.9 \mathrm{~Hz}, 1 \mathrm{H}), 6.00$ (d, $J=2.3 \mathrm{~Hz}, 1 \mathrm{H}), 2.67$ (t, $J=7.4 \mathrm{~Hz}, 2 \mathrm{H}), 2.27$ (m, 2H), 2.24 (s, $3 \mathrm{H}$ ), 1.83 (quint, $J=7.4 \mathrm{~Hz}, 2 \mathrm{H}$ ) ppm; ${ }^{13} \mathrm{C} \mathrm{NMR} \mathrm{(125} \mathrm{MHz,} \mathrm{CDCl}_{3}$ ): 198.4, 155.1, 147.3, 140.8, 131.5, 110.0, 105.1, 31.6, 27.2, 26.7, 26.4 ppm.

\section{(E)-6-(furan-2-yl)-1-phenylhex-2-en-1-one (1c)}

The reaction was accomplished according to the general
experimental procedure described above. Product 1c was purified by flash column chromatography (silica gel, petroleum ether : EtOAc $=70: 1)$. Yield $192 \mathrm{mg}(80 \%)$ of a yellow oil. ${ }^{1} \mathrm{H}$ NMR $(500 \mathrm{MHz}$, $\left.\mathrm{CDCl}_{3}\right): 7.88(\mathrm{~d}, J=7.4 \mathrm{~Hz}, 2 \mathrm{H}), 7.49$ (t, $\left.J=7.4 \mathrm{~Hz}, 1 \mathrm{H}\right), 7.40$ (t, $\left.J=7.4 \mathrm{~Hz}, 2 \mathrm{H}\right), 7.26$ $(\mathrm{m}, 1 \mathrm{H}), 7.01\left(\mathrm{dt}, J_{l}=15.4 \mathrm{~Hz}, J_{2}=7.0 \mathrm{~Hz}, 1 \mathrm{H}\right), 6.85(\mathrm{~d}, J=15.4 \mathrm{~Hz}, 1 \mathrm{H}), 6.24(\mathrm{~m}, 1 \mathrm{H})$, $5.97(\mathrm{~m}, 1 \mathrm{H}), 2.64(\mathrm{t}, J=7.4 \mathrm{~Hz}, 2 \mathrm{H}), 2.30(\mathrm{q}, J=7.0 \mathrm{~Hz}, 2 \mathrm{H}), 1.83$ (quint, $J=7.4 \mathrm{~Hz}$, 2H) ppm; ${ }^{13} \mathrm{C}$ NMR $\left(125 \mathrm{MHz}, \mathrm{CDCl}_{3}\right): 190.5,155.1,148.7,140.8,137.7,132.5$, 128.4 (4C), 126.1, 110.0, 105.1, 31.9, 27.2, 26.4 ppm; HRMS (Orbitrap ESI): calcd for $\mathrm{C}_{16} \mathrm{H}_{17} \mathrm{O}_{2}: 241.1223[\mathrm{M}+\mathrm{H}]^{+}$; found 241.1220.

\section{Methyl $(E)-6$-(furan-2-yl)hex-2-enoate $(18)^{7}$}

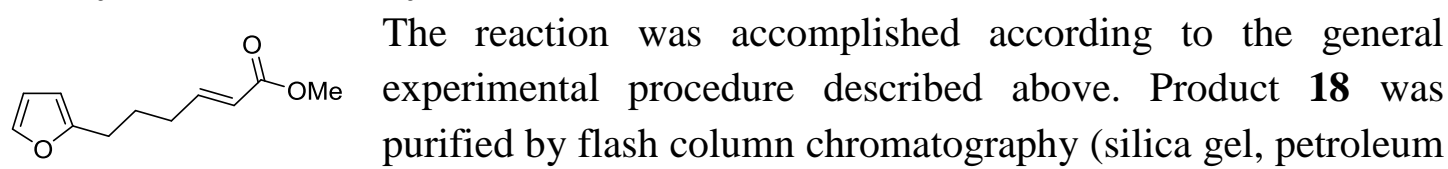
ether : EtOAc $=70: 1)$. Yield $728 \mathrm{mg}(75 \%)$ of a yellow oil. ${ }^{1} \mathrm{H}$ NMR $(500 \mathrm{MHz}$, $\left.\mathrm{CDCl}_{3}\right): 7.30\left(\mathrm{dd}, J_{l}=1.8 \mathrm{~Hz}, J_{2}=0.8 \mathrm{~Hz}, 1 \mathrm{H}\right), 6.96\left(\mathrm{dt}, J_{l}=15.7 \mathrm{~Hz}, J_{2}=7.0 \mathrm{~Hz}, 1 \mathrm{H}\right)$, $6.28\left(\mathrm{dd}, J_{l}=3.1 \mathrm{~Hz}, J_{2}=1.8 \mathrm{~Hz}, 1 \mathrm{H}\right), 5.99\left(\mathrm{dd}, J_{l}=3.1 \mathrm{~Hz}, J_{2}=0.8 \mathrm{~Hz}, 1 \mathrm{H}\right), 5.84(\mathrm{dt}$, $\left.J_{l}=15.7 \mathrm{~Hz}, J_{2}=1.5 \mathrm{~Hz}, 1 \mathrm{H}\right), 3.73(\mathrm{~s}, 3 \mathrm{H}), 2.65(\mathrm{t}, J=7.4 \mathrm{~Hz}, 2 \mathrm{H}), 2.24\left(\mathrm{qd}, J_{l}=7.0 \mathrm{~Hz}\right.$, $J_{2}=1.5 \mathrm{~Hz}, 2 \mathrm{H}$ ), 1.81 (quint, $J=7.4 \mathrm{~Hz}, 2 \mathrm{H}$ ) ppm; ${ }^{13} \mathrm{C} \mathrm{NMR}\left(125 \mathrm{MHz}, \mathrm{CDCl}_{3}\right.$ ): 167.0, $155.3,148.7,141.0,121.4,110.1,105.2,51.4,31.4,27.2,26.4$ ppm.

\section{(E)-8-(furan-2-yl)oct-3-en-2-one (2b)}

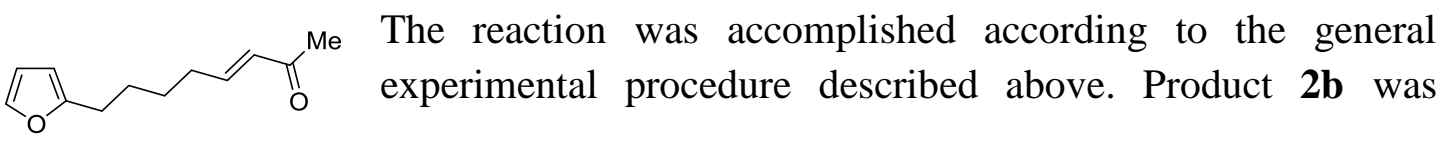

\footnotetext{
${ }^{6}$ Chung, W. K.; Lam, S. K.; Lo, B.; Liu, L. L.; Wong, W.-T.; Chiu, P. J. Am. Chem. Soc. 2009, 131, 4556-4557.

${ }^{7}$ Riedel, S.; Maichle-Mössmer, C.; Maier, M. E. J. Org. Chem. 2017, 82, 12798-12805.
} 
purified by flash column chromatography (silica gel, petroleum ether : EtOAc $=$ 70:1). Yield $78.3 \mathrm{mg}(80 \%)$ of a yellow oil. ${ }^{1} \mathrm{H}$ NMR $\left(500 \mathrm{MHz}, \mathrm{CDCl}_{3}\right): 7.29(\mathrm{~m}$, $1 \mathrm{H}), 6.78\left(\mathrm{dt}, J_{l}=15.9 \mathrm{~Hz}, J_{2}=6.8 \mathrm{~Hz}, 1 \mathrm{H}\right), 6.28\left(\mathrm{dd}, J_{l}=3.0 \mathrm{~Hz}, J_{2}=2.0 \mathrm{~Hz}, 1 \mathrm{H}\right), 6.07$ (d, J=15.9 Hz, 1H), 5.98 (d, J=3.0 Hz, 1H), $2.64(\mathrm{t}, J=7.4 \mathrm{~Hz}, 2 \mathrm{H}), 2.25(\mathrm{~m}, 2 \mathrm{H}), 2.24$ (s, 3H), 1.67 (quint, $J=7.4 \mathrm{~Hz}, 2 \mathrm{H}$ ), 1.52 (quint, $J=7.4 \mathrm{~Hz}, 2 \mathrm{H}) \mathrm{ppm} ;{ }^{13} \mathrm{C} \mathrm{NMR}(125$ $\mathrm{MHz}, \mathrm{CDCl}_{3}$ ): 198.6, 155.8, 147.9, 140.8, 131.4, 110.1, 104.9, 32.1, 27.7, 27.6, 27.5, 26.9 ppm; HRMS (Orbitrap ESI): calcd for $\mathrm{C}_{12} \mathrm{H}_{17} \mathrm{O}_{2}: 193.1223[\mathrm{M}+\mathrm{H}]^{+}$; found 193.1221 .

Methyl (E)-7-(furan-2-yl)hept-2-enoate (19) ${ }^{7}$

The reaction was accomplished according to the general
experimental procedure described above. Product 19 was
purified by flash column chromatography (silica gel, petroleum ether : EtOAc $=80: 1)$. Yield $320 \mathrm{mg}(85 \%)$ of yellow oil. ${ }^{1} \mathrm{H}$ NMR $(500$ $\mathrm{MHz}, \mathrm{CDCl}_{3}$ ): $7.29\left(\mathrm{dd}, J_{l}=1.8 \mathrm{~Hz}, J_{2}=0.7 \mathrm{~Hz}, 1 \mathrm{H}\right), 6.96\left(\mathrm{dt}, J_{l}=15.7 \mathrm{~Hz}, J_{2}=7.0 \mathrm{~Hz}\right.$, $1 \mathrm{H}), 6.27\left(\mathrm{dd}, J_{l}=3.1 \mathrm{~Hz}, J_{2}=1.8 \mathrm{~Hz}, 1 \mathrm{H}\right), 5.97\left(\mathrm{dd}, J_{l}=3.1 \mathrm{~Hz}, J_{2}=0.7 \mathrm{~Hz}, 1 \mathrm{H}\right), 5.82$ $\left(\mathrm{dt}, J_{l}=15.7 \mathrm{~Hz}, J_{2}=1.6 \mathrm{~Hz}, 1 \mathrm{H}\right), 3.72(\mathrm{~s}, 3 \mathrm{H}), 2.63(\mathrm{t}, J=7.4 \mathrm{~Hz}, 2 \mathrm{H}), 2.23\left(\mathrm{qd}, J_{l}=7.0\right.$ $\mathrm{Hz}, J_{2}=1.6 \mathrm{~Hz}, 2 \mathrm{H}$ ), 1.67 (quint, $J=7.4 \mathrm{~Hz}, 2 \mathrm{H}$ ), 1.51 (quint, $J=7.4 \mathrm{~Hz}, 2 \mathrm{H}$ ) ppm; ${ }^{13} \mathrm{C}$ NMR (125 MHz, $\left.\mathrm{CDCl}_{3}\right)$ : 167.1, 155.8, 149.2, 140.8, 121.1, 110.0, 104.8, 51.4, 31.9, 27.7, 27.5, $27.4 \mathrm{ppm}$.

\section{(E)-7-(furan-2-yl)-1-phenylhept-2-en-1-one (2c)}

The reaction was accomplished according to the general
experimental procedure described above. Product 2c was purified by flash column chromatography (silica gel, petroleum ether : EtOAc $=90: 1)$. Yield $416 \mathrm{mg}(91 \%)$ of a yellow oil. ${ }^{1} \mathrm{H}$ NMR $(500 \mathrm{MHz}$, $\mathrm{CDCl}_{3}$ ): 7.92 (d, J=7.3 Hz, 2H), 7.55 (t, J=7.3 Hz, 1H), 7.47 (t, J=7.6 Hz, 2H), 7.30 $(\mathrm{m}, 1 \mathrm{H}), 7.05\left(\mathrm{dt}, J_{l}=15.4 \mathrm{~Hz}, J_{2}=7.0 \mathrm{~Hz}, 1 \mathrm{H}\right), 6.87(\mathrm{~d}, J=15.4 \mathrm{~Hz}, 1 \mathrm{H}), 6.28(\mathrm{dd}$, $\left.J_{l}=3.0 \mathrm{~Hz}, J_{2}=2.8 \mathrm{~Hz}, 1 \mathrm{H}\right), 5.99(\mathrm{~d}, J=3.0 \mathrm{~Hz}, 1 \mathrm{H}), 2.66(\mathrm{t}, J=7.4 \mathrm{~Hz}, 2 \mathrm{H}), 2.35$ (q, $J=6.8 \mathrm{~Hz}, 2 \mathrm{H}$ ), 1.72 (quint, $J=7.4 \mathrm{~Hz}, 2 \mathrm{H}$ ), 1.59 (quint, $J=7.4 \mathrm{~Hz}, 2 \mathrm{H}$ ) ppm; ${ }^{13} \mathrm{C} \mathrm{NMR}$ (125 MHz, $\left.\mathrm{CDCl}_{3}\right): 190.9,155.8,149.5,140.8,138.0,132.6,128.5$ (4C), 126.1, 110.1, 104.9, 32.5, 27.7, 27.6 (2C) ppm; HRMS (Orbitrap ESI): calcd for $\mathrm{C}_{17} \mathrm{H}_{19} \mathrm{O}_{2}$ : $255.1380[\mathrm{M}+\mathrm{H}]^{+}$; found 255.1377 .

\section{Synthesis of alcohols 20 and 21}

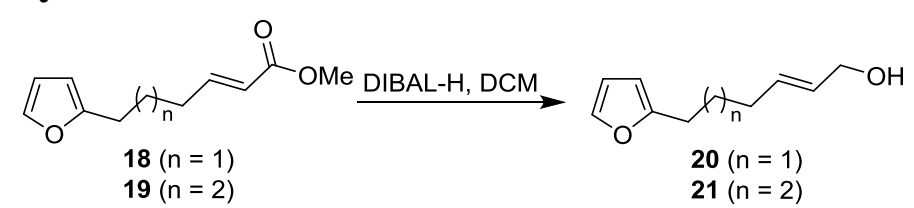

To a stirred solution of the corresponding unsaturated ester $(728 \mathrm{mg}, 3.75 \mathrm{mmol}$ for 18, $320 \mathrm{mg}, 1.54 \mathrm{mmol}$ for 19$)$ in dry $\mathrm{CH}_{2} \mathrm{Cl}_{2}(12 \mathrm{~mL}$ for $18,5 \mathrm{~mL}$ for 19$)$, at $-78{ }^{\circ} \mathrm{C}$ and under an argon atmosphere, was added dropwise DIBAL-H (1.0 M solution in hexane, $8.75 \mathrm{~mL}, 8.75 \mathrm{mmol}$ towards $\mathbf{2 0}, 3.6 \mathrm{~mL}, 3.6 \mathrm{mmol}$ towards 21 ). The mixture was maintained at this temperature for $2 \mathrm{~h}$ until full consumption of the starting ester 
was confirmed by tlc analysis. Then the solution was poured directly into a saturated aqueous potassium sodium tartrate solution $(10 \mathrm{~mL}$ towards to $\mathbf{2 0}, 5 \mathrm{~mL}$ towards to 21) and stirred for $1 \mathrm{~h}$ at $\mathrm{rt}$. The layers were separated and the aqueous layer was extracted with $\mathrm{CH}_{2} \mathrm{Cl}_{2}(2 \times 10 \mathrm{~mL}$ towards $\mathbf{2 0}, 2 \times 5 \mathrm{~mL}$ towards 21). The combined organic layers were dried with $\mathrm{Na}_{2} \mathrm{SO}_{4}$ and concentrated in vacuo. The residue was purified by flash column chromatography (silica gel, petroleum ether : EtOAc).

\section{(E)-6-(furan-2-yl)hex-2-en-1-ol (20) ${ }^{8}$}

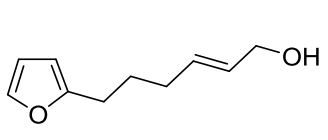

The reaction was accomplished according to the general experimental procedure described above. Product $\mathbf{2 0}$ was purified by flash column chromatography (silica gel, petroleum ether : EtOAc $=6: 1)$. Yield $488 \mathrm{mg}(78 \%)$ of a slightly yellow oil. ${ }^{1} \mathrm{H}$ NMR (500 $\mathrm{MHz}, \mathrm{CDCl}_{3}$ ): $7.30\left(\mathrm{dd}, J_{l}=1.8 \mathrm{~Hz}, J_{2}=0.8 \mathrm{~Hz}, 1 \mathrm{H}\right), 6.28\left(\mathrm{dd}, J_{l}=3.1 \mathrm{~Hz}, J_{2}=1.8 \mathrm{~Hz}\right.$, $1 \mathrm{H}), 5.98\left(\mathrm{dd}, J_{l}=3.1 \mathrm{~Hz}, J_{2}=0.8 \mathrm{~Hz}, 1 \mathrm{H}\right), 5.67(\mathrm{~m}, 2 \mathrm{H}), 4.08(\mathrm{~m}, 2 \mathrm{H}), 2.63(\mathrm{t}, J=7.4$ $\mathrm{Hz}, 2 \mathrm{H}), 2.10(\mathrm{~m}, 2 \mathrm{H}), 1.74$ (quint, $J=7.4 \mathrm{~Hz}, 2 \mathrm{H}$ ), 1.30 (brs, $1 \mathrm{H}$ ) ppm; ${ }^{13} \mathrm{C} \mathrm{NMR}$ (125 MHz, $\left.\mathrm{CDCl}_{3}\right)$ : 156.0, 140.7, 132.4, 129.6, 110.0, 104.8, 63.7, 31.5, 27.4, 27.3 ppm.

\section{(E)-7-(furan-2-yl)hept-2-en-1-ol (21)}

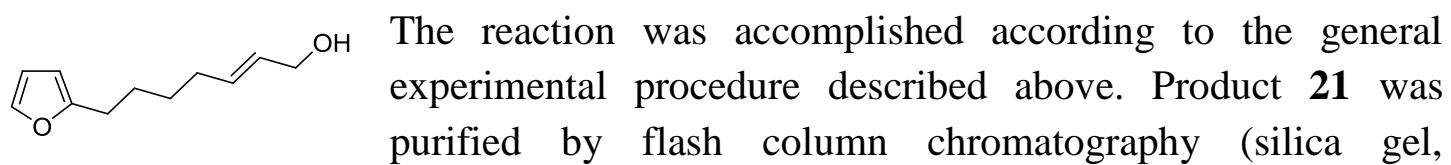
petroleum ether : EtOAc $=6: 1)$. Yield $228 \mathrm{mg}(82 \%)$ of a slightly yellow oil. ${ }^{1} \mathrm{H}$ NMR (500 MHz, $\left.\mathrm{CDCl}_{3}\right): 7.29\left(\mathrm{dd}, J_{l}=1.8 \mathrm{~Hz}, J_{2}=0.8 \mathrm{~Hz}, 1 \mathrm{H}\right), 6.27\left(\mathrm{dd}, J_{l}=3.1 \mathrm{~Hz}\right.$, $\left.J_{2}=1.8 \mathrm{~Hz}, 1 \mathrm{H}\right), 5.97\left(\mathrm{dd}, J_{1}=3.1 \mathrm{~Hz}, J_{2}=0.8 \mathrm{~Hz}, 1 \mathrm{H}\right), 5.66(\mathrm{~m}, 2 \mathrm{H}), 4.09(\mathrm{t}, J=5.3 \mathrm{~Hz}$, $2 \mathrm{H}), 2.62(\mathrm{t}, J=7.5 \mathrm{~Hz}, 2 \mathrm{H}), 2.08\left(\mathrm{td}, J_{l}=7.5 \mathrm{~Hz}, J_{2}=6.8 \mathrm{~Hz}, 2 \mathrm{H}\right), 1.65$ (quint, $J=7.5$ $\mathrm{Hz}, 2 \mathrm{H}$ ), 1.44 (quint, $J=7.5 \mathrm{~Hz}, 2 \mathrm{H}$ ) ppm; ${ }^{13} \mathrm{C} \mathrm{NMR}\left(125 \mathrm{MHz}, \mathrm{CDCl}_{3}\right.$ ): 156.2, 140.7, 133.0, 129.1, 110.0, 104.7, 63.8, 31.9, 28.6, 27.8, 27.5 ppm; HRMS (Orbitrap ESI): calcd for $\mathrm{C}_{11} \mathrm{H}_{17} \mathrm{O}_{2}: 181.1223[\mathrm{M}+\mathrm{H}]^{+}$; found 181.1223 .

\section{Synthesis of aldehydes $1 \mathrm{a}$ and $2 \mathrm{a}$}

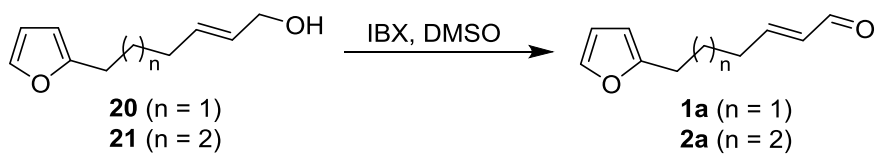

2-Iodoxybenzoic acid $(1.65 \mathrm{~g}, 5.88 \mathrm{mmol}$ towards 1a, $709 \mathrm{mg}, 2.53 \mathrm{mmol}$ towards 2a) in dry DMSO (19.0 $\mathrm{mL}$ for $\mathbf{2 0}, 8.0 \mathrm{~mL}$ for $\mathbf{2 1})$ at $\mathrm{rt}$ was stirred until the mixture became transparent. A solution of the corresponding alcohol (488 mg, $2.94 \mathrm{mmol}$ for 20, $228 \mathrm{mg}, 1.27 \mathrm{mmol}$ for 21) in dry DMSO ( $1 \mathrm{~mL}$ for 20, $0.5 \mathrm{~mL}$ for 21) was added slowly and the reaction was stirred at $\mathrm{rt}$. After completion of the reaction, as was indicated by tlc analysis $(2 \mathrm{~h}), \mathrm{CH}_{2} \mathrm{Cl}_{2}(15 \mathrm{~mL}$ towards $\mathbf{1 a}, 7 \mathrm{~mL}$ towards $2 \mathbf{a})$ was added. The mixture was cooled to $0{ }^{\circ} \mathrm{C}$ using an ice bath and distilled water $(10 \mathrm{~mL}$ towards 1a, $5 \mathrm{~mL}$ towards $\mathbf{2 a}$ ) was added. After the precipitation of solids, the

\footnotetext{
${ }^{8}$ Fillion, E.; Beingessner, R. L. J. Org. Chem. 2003, 68, 9485-9488.
} 
mixture was filtered through cotton wool and the layers of the resulting biphasic solution separated. The organic phase was washed with a saturated aqueous solution of $\mathrm{NaHCO}_{3}(3 \times 10 \mathrm{~mL}$ towards $\mathbf{1 a}, 3 \times 5 \mathrm{~mL}$ towards $\mathbf{2 a})$ followed by brine $(10 \mathrm{~mL}$ towards $1 \mathbf{a}, 5 \mathrm{~mL}$ towards $\mathbf{2 a}$ ). The organic phase was dried over $\mathrm{Na}_{2} \mathrm{SO}_{4}$, filtered and concentrated in vacuo. The residue was purified by flash column chromatography (silica gel, petroleum ether : EtOAc)

\section{(E)-6-(furan-2-yl)hex-2-enal (1a)}

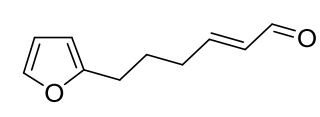

The reaction was accomplished according to the general experimental procedure described above. Product 1a was purified by flash column chromatography (silica gel, petroleum ether : EtOAc $=40: 1)$. Yield $429 \mathrm{mg}(89 \%)$ of a yellow oil. ${ }^{1} \mathrm{H} \mathrm{NMR}\left(500 \mathrm{MHz}, \mathrm{CDCl}_{3}\right)$ : $9.50(\mathrm{~d}, J=7.9 \mathrm{~Hz}, 1 \mathrm{H}), 7.31(\mathrm{~d}, J=1.8 \mathrm{~Hz}, 1 \mathrm{H}), 6.82\left(\mathrm{dt}, J_{l}=15.7 \mathrm{~Hz}, J_{2}=6.8 \mathrm{~Hz}, 1 \mathrm{H}\right)$, $6.28\left(\mathrm{dd}, J_{l}=3.0 \mathrm{~Hz}, J_{2}=1.8 \mathrm{~Hz}, 1 \mathrm{H}\right), 6.12\left(\mathrm{ddt}, J_{l}=15.7 \mathrm{~Hz}, J_{2}=7.9 \mathrm{~Hz}, J_{3}=1.4 \mathrm{~Hz}\right.$, $1 \mathrm{H}), 6.00(\mathrm{~d}, J=3.0 \mathrm{~Hz}, 1 \mathrm{H}), 2.69(\mathrm{t}, J=7.4 \mathrm{~Hz}, 2 \mathrm{H}), 2.38(\mathrm{~m}, 2 \mathrm{H}), 1.87$ (quint, $J=7.4$ $\mathrm{Hz}, 2 \mathrm{H}) \mathrm{ppm} ;{ }^{13} \mathrm{C} \mathrm{NMR}\left(125 \mathrm{MHz}, \mathrm{CDCl}_{3}\right): 193.9,157.8,154.9,141.0,133.3,110.1$, 105.4, 31.9, 27.3, 26.3 ppm; HRMS (Orbitrap ESI): calcd for $\mathrm{C}_{10} \mathrm{H}_{13} \mathrm{O}_{2}: 165.0910$ $[\mathrm{M}+\mathrm{H}]^{+}$; found 165.0910 .

\section{(E)-7-(furan-2-yl)hept-2-enal (2a)}

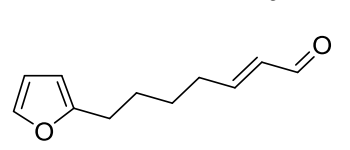

The reaction was accomplished according to the general experimental procedure described above. Product 2a was purified by flash column chromatography (silica gel, petroleum ether : EtOAc $=50: 1)$. Yield $210 \mathrm{mg}(93 \%)$ of a yellow oil. ${ }^{1} \mathrm{H}$ NMR $(500 \mathrm{MHz}$, $\left.\mathrm{CDCl}_{3}\right): 9.50(\mathrm{~d}, J=7.9 \mathrm{~Hz}, 1 \mathrm{H}), 7.30\left(\mathrm{dd}, J_{l}=1.8 \mathrm{~Hz}, J_{2}=0.8 \mathrm{~Hz}, 1 \mathrm{H}\right), 6.83(\mathrm{dt}$, $\left.J_{l}=15.6 \mathrm{~Hz}, J_{2}=6.8 \mathrm{~Hz}, 1 \mathrm{H}\right), 6.28\left(\mathrm{dd}, J_{l}=3.1 \mathrm{~Hz}, J_{2}=1.8 \mathrm{~Hz}, 1 \mathrm{H}\right), 6.12\left(\mathrm{ddt}, J_{l}=15.6\right.$ $\left.\mathrm{Hz}, J_{2}=7.9 \mathrm{~Hz}, J_{3}=1.5 \mathrm{~Hz}, 1 \mathrm{H}\right), 5.98\left(\mathrm{dd}, J_{l}=3.1 \mathrm{~Hz}, J_{2}=0.8 \mathrm{~Hz}, 1 \mathrm{H}\right), 2.66(\mathrm{t}, J=7.4$ $\mathrm{Hz}, 2 \mathrm{H}$ ), 2.36 (m, 2H), 1.70 (quint, $J=7.4 \mathrm{~Hz}, 2 \mathrm{H}$ ), 1.56 (quint, $J=7.4 \mathrm{~Hz}, 2 \mathrm{H}$ ) ppm; ${ }^{13} \mathrm{C}$ NMR (125 MHz, $\mathrm{CDCl}_{3}$ ): 194.0, 158.3, 155.6, 140.9, 133.1, 110.1, 105.0, 32.4, 27.6, 27.5, 27.2 ppm; HRMS (Orbitrap ESI): calcd for $\mathrm{C}_{11} \mathrm{H}_{15} \mathrm{O}_{2}: 179.1067[\mathrm{M}+\mathrm{H}]^{+}$; found 179.1066 . 


\section{General experimental procedure for the preparation of 1-azaspirocyclic compounds of type 3 and 4 from furans.}

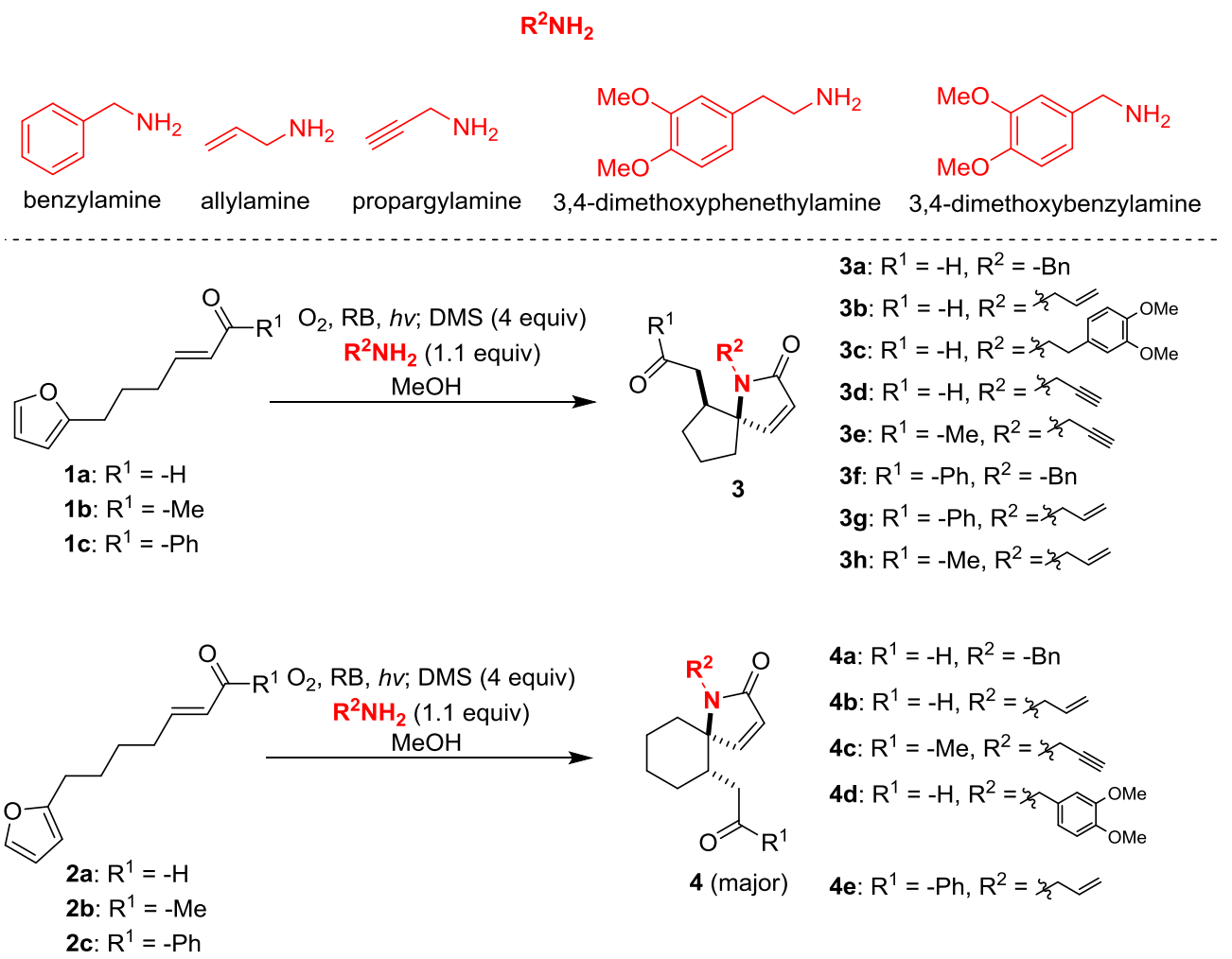

2-Substituted furan ( $0.2 \mathrm{mmol}, 32.8 \mathrm{mg}$ for $\mathbf{1 a}, 35.6 \mathrm{mg}$ for $\mathbf{1 b}, 48 \mathrm{mg}$ for $\mathbf{1 c}, 35.6 \mathrm{mg}$ for $\mathbf{2 a}, 38.4 \mathrm{mg}$ for $\mathbf{2 b}, 50.8 \mathrm{mg}$ for $\mathbf{2 c}$ ) was dissolved in $\mathrm{MeOH}(4 \mathrm{~mL}, 50 \mathrm{mM}$ ) containing catalytic amounts $(1 \% \mathrm{~mol}, 2 \mathrm{mg})$ of rose Bengal as photosensitizer. The solution was cooled with an ice bath. Oxygen was gently bubbled through the solutions while they were irradiated with a xenon Variac Eimac Cermax 300 W lamp. After completion of the reaction $(2 \mathrm{~min})$, the solution was warmed to room temperature and $\mathrm{Me}_{2} \mathrm{~S}(58 \mu \mathrm{L}, 0.8 \mathrm{mmol}$ ) was added. After completion of the reduction, as was indicated by tlc analysis $(45 \mathrm{~min})$, the corresponding amine $(0.22$ mmol, $24 \mu \mathrm{L}$ for benzylamine, $16.5 \mu \mathrm{L}$ for allylamine, $14.1 \mu \mathrm{L}$ for propargylamine, $37.1 \mu \mathrm{L}$ for 3,4-dimethoxyphenethylamine, $33.2 \mu \mathrm{L}$ for 3,4-dimethoxybenzylamine) was added and the reaction stirred at $\mathrm{rt}$. The reaction was monitored by tlc analysis and by ${ }^{1} \mathrm{H}-\mathrm{NMR}$. After completion of the reaction ( $2 \mathrm{~h}$ towards products of type 3 only in case of the reaction towards $3 \mathbf{e}$ was the reaction left for $5 \mathrm{~h}$, or $24 \mathrm{~h}$ towards products of type 4) the solvent was removed in vacuo and the residue was purified by flash column chromatography (silica gel, petroleum ether : EtOAc). For all the spirocycles of type 3 where the diastereoselectivity was high (7/1-9/1), but also in the case of $\mathbf{4 c}$, the major diastereoisomer was isolated in a pure form, after column chromatography and the isolated yields refer to the major purified diastereoisomer. In the case of $\mathbf{4 e}$, the two diastereoisomers were separable and were isolated in a pure form after column chromatography. Finally, in the cases of $\mathbf{4 a}, \mathbf{4 b}$ and $\mathbf{4 d}$ the two diastereoisomers were inseparable and the combined yield (after column chromatography) is reported. 
The reaction was scaled up to $1 \mathrm{mmol}$ of starting furans $\mathbf{1 a}$ and $\mathbf{2 c}$ for the preparation of compounds $\mathbf{3 a}$ and $\mathbf{4 e}$. The isolated yield for the major diastereoisomer of $\mathbf{3 a}$ was $66 \%(177 \mathrm{mg})$ and the isolated combined yield for the two separable diastereoisomers of $4 \mathbf{e}$ was $59 \%(183 \mathrm{mg})$. Finally, when the reaction was scaled up to $3 \mathrm{mmol}$ of starting furan 1a for the preparation of compounds $\mathbf{3 a}$ the isolated yield for the major diastereoisomer was $63 \%(510 \mathrm{mg})$.

\section{2-(1-benzyl-2-oxo-1-azaspiro[4.4]non-3-en-6-yl)acetaldehyde (3a)}

Bn $O$ The product was prepared according to the general experimental procedure described above as a $9 / 1$ mixture of separable diastereoisomers. The crude product was purified by flash column chromatography (silica gel, petroleum ether : EtOAc $=1: 1$ ) to furnish 3a as a brown oil (yield $=35.5 \mathrm{mg}, 66 \%)$.

${ }^{1} \mathrm{H}$ NMR (500 MHz, $\mathrm{CDCl}_{3}$ ): 9.62 (s, 1H), 7.31-7.22 (m, 5H), 6.82 (d, J=5.8 Hz, 1H), $6.16(\mathrm{~d}, J=5.8 \mathrm{~Hz}, 1 \mathrm{H}), 4.75(\mathrm{~d}, J=15.9 \mathrm{~Hz}, 1 \mathrm{H}), 4.39(\mathrm{~d}, J=15.9 \mathrm{~Hz}, 1 \mathrm{H}), 2.61(\mathrm{~m}$, $1 \mathrm{H}), 2.19-2.06(\mathrm{~m}, 3 \mathrm{H}), 2.04\left(\mathrm{ddd}, J_{l}=18.3 \mathrm{~Hz}, J_{2}=9.5 \mathrm{~Hz}, J_{3}=1.3 \mathrm{~Hz}, 1 \mathrm{H}\right), 1.85(\mathrm{~m}$, $1 \mathrm{H}), 1.80-1.72(\mathrm{~m}, 2 \mathrm{H}), 1.55(\mathrm{~m}, 1 \mathrm{H}) \mathrm{ppm} ;{ }^{13} \mathrm{C} \mathrm{NMR}\left(125 \mathrm{MHz}, \mathrm{CDCl}_{3}\right): 200.5$, 172.2, 153.3, 138.2, 128.5 (2C), 127.3 (3C), 125.1, 77.3, 45.0, 42.6, 41.0, 33.1, 31.9, 23.2 ppm; HRMS (Orbitrap ESI): calcd for $\mathrm{C}_{17} \mathrm{H}_{20} \mathrm{NO}_{2}: 270.1489[\mathrm{M}+\mathrm{H}]^{+}$; found 270.1489 .

\section{2-(1-allyl-2-oxo-1-azaspiro[4.4]non-3-en-6-yl)acetaldehyde (3b)}

The product was prepared according to the general experimental

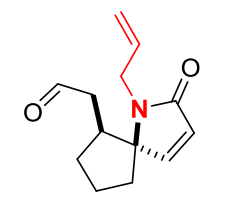
procedure described above as a 7.5/1 mixture of separable diastereoisomers. The crude product was purified by flash column chromatography (silica gel, petroleum ether : EtOAc $=1: 1$ ) to furnish $\mathbf{3 b}$ as a brown oil (yield $=26.7 \mathrm{mg}, 61 \%$ ).

${ }^{1} \mathrm{H}$ NMR (500 MHz, $\left.\mathrm{CDCl}_{3}\right): 9.70(\mathrm{t}, J=1.0 \mathrm{~Hz}, 1 \mathrm{H}), 6.79$ (d, J=5.8 Hz, 1H), 6.06 (d, $J=5.8 \mathrm{~Hz}, 1 \mathrm{H}), 5.89$ (ddt, $\left.J_{l}=17.3 \mathrm{~Hz}, J_{2}=10.3 \mathrm{~Hz}, J_{3}=5.7 \mathrm{~Hz}, 1 \mathrm{H}\right), 5.15(\mathrm{~m}, 2 \mathrm{H}), 4.13$ (ddt, $\left.J_{l}=16.1 \mathrm{~Hz}, J_{2}=5.7 \mathrm{~Hz}, J_{3}=1.5 \mathrm{~Hz}, 1 \mathrm{H}\right), 3.78\left(\mathrm{ddt}, J_{l}=16.1 \mathrm{~Hz}, J_{2}=5.7 \mathrm{~Hz}, J_{3}=1.5\right.$ $\mathrm{Hz}, 1 \mathrm{H}), 2.62(\mathrm{~m}, 1 \mathrm{H}), 2.28\left(\mathrm{ddd}, J_{l}=18.1 \mathrm{~Hz}, J_{2}=8.9 \mathrm{~Hz}, J_{3}=1.4 \mathrm{~Hz}, 1 \mathrm{H}\right), 2.24-2.14$ $(\mathrm{m}, 3 \mathrm{H}), 2.04-1.91(\mathrm{~m}, 2 \mathrm{H}), 1.85(\mathrm{~m}, 1 \mathrm{H}), 1.58(\mathrm{~m}, 1 \mathrm{H}) \mathrm{ppm} ;{ }^{13} \mathrm{C}$ NMR $(125 \mathrm{MHz}$, $\left.\mathrm{CDCl}_{3}\right): 200.4,171.3,152.9,134.2,125.1,116.5,76.9,44.3,42.7,40.9,33.1,32.0$, 23.2 ppm; HRMS (Orbitrap ESI): calcd for $\mathrm{C}_{13} \mathrm{H}_{18} \mathrm{NO}_{2}$ : $220.1332[\mathrm{M}+\mathrm{H}]^{+}$; found 220.1334 .

\section{2-(1-(3,4-dimethoxyphenethyl)-2-oxo-1-azaspiro[4.4]non-3-en-6-yl)acetaldehyde}

(3c)

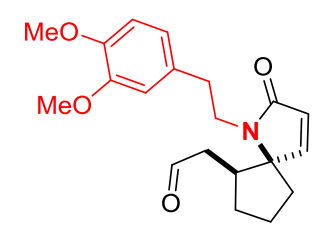

The product was prepared according to the general experimental procedure described above as a 7/1 mixture of separable diastereoisomers. The crude product was purified by flash column chromatography (silica gel, petroleum ether : EtOAc $=1: 3$ ) to furnish $3 \mathbf{c}$ as a brown oil (yield $=41.2 \mathrm{mg}, 60 \%$ ). 
${ }^{1} \mathrm{H}$ NMR (500 MHz, $\left.\mathrm{CDCl}_{3}\right): 9.68(\mathrm{~s}, 1 \mathrm{H}), 6.78(\mathrm{~m}, 4 \mathrm{H}), 6.06(\mathrm{~d}, J=5.8 \mathrm{~Hz}, 1 \mathrm{H}), 3.87$ $(\mathrm{s}, 3 \mathrm{H}), 3.85(\mathrm{~s}, 3 \mathrm{H}), 3.72\left(\mathrm{ddd}, J_{l}=13.8 \mathrm{~Hz}, J_{2}=10.8 \mathrm{~Hz}, J_{3}=5.0 \mathrm{~Hz}, 1 \mathrm{H}\right), 3.20$ (ddd, $\left.J_{l}=13.8 \mathrm{~Hz}, J_{2}=10.5 \mathrm{~Hz}, J_{3}=6.2 \mathrm{~Hz}, 1 \mathrm{H}\right), 3.01\left(\mathrm{ddd}, J_{l}=13.0 \mathrm{~Hz}, J_{2}=10.5 \mathrm{~Hz}, J_{3}=5.0\right.$ $\mathrm{Hz}, 1 \mathrm{H}), 2.89\left(\mathrm{ddd}, J_{l}=13.0 \mathrm{~Hz}, J_{2}=10.8 \mathrm{~Hz}, J_{3}=6.2 \mathrm{~Hz}, 1 \mathrm{H}\right), 2.65(\mathrm{~m}, 1 \mathrm{H}), 2.28(\mathrm{~m}$, $1 \mathrm{H}), 2.22(\mathrm{~m}, 2 \mathrm{H}), 2.12\left(\mathrm{ddd}, J_{1}=14.4 \mathrm{~Hz}, J_{2}=10.6 \mathrm{~Hz}, J_{3}=7.0 \mathrm{~Hz}, 1 \mathrm{H}\right), 2.01(\mathrm{~m}, 1 \mathrm{H})$, $1.89(\mathrm{~m}, 1 \mathrm{H}), 1.74\left(\mathrm{ddd}, J_{l}=14.4 \mathrm{~Hz}, J_{2}=8.7 \mathrm{~Hz}, J_{3}=5.5 \mathrm{~Hz}, 1 \mathrm{H}\right), 1.56(\mathrm{~m}, 1 \mathrm{H}) \mathrm{ppm}$; ${ }^{13} \mathrm{C}$ NMR (125 MHz, $\mathrm{CDCl}_{3}$ ): 200.3, 171.7, 152.7, 149.0, 147.7, 131.6, 125.5, 120.6, 112.1, 111.3, 76.7, 55.9 (2C), 45.0, 42.9, 40.9, 34.7, 33.4, 32.3, 23.6 ppm; HRMS (Orbitrap ESI): calcd for $\mathrm{C}_{20} \mathrm{H}_{26} \mathrm{NO}_{4}$ : $344.1856[\mathrm{M}+\mathrm{H}]^{+}$; found 344.1858 .

\section{2-(2-oxo-1-(prop-2-yn-1-yl)-1-azaspiro[4.4]non-3-en-6-yl)acetaldehyde (3d)}

The product was prepared according to the general experimental
diastereoisomers. The crude product was purified by flash column
chromatography (silica gel, petroleum ether : EtOAc $=1: 2$ ) to furnish 3d as a brown oil (yield $=23.9 \mathrm{mg}, 55 \%$ ).

${ }^{1} \mathrm{H}$ NMR (500 MHz, $\left.\mathrm{CDCl}_{3}\right): 9.71(\mathrm{~s}, 1 \mathrm{H}), 6.83(\mathrm{~d}, J=5.9 \mathrm{~Hz}, 1 \mathrm{H}), 6.09$ (d, J=5.9 Hz, $1 \mathrm{H}), 4.20\left(\mathrm{dd}, J_{l}=17.8 \mathrm{~Hz}, J_{2}=2.5 \mathrm{~Hz}, 1 \mathrm{H}\right), 4.07\left(\mathrm{dd}, J_{l}=17.8 \mathrm{~Hz}, J_{2}=2.5 \mathrm{~Hz}, 1 \mathrm{H}\right)$, $2.65(\mathrm{~m}, 1 \mathrm{H}), 2.51\left(\mathrm{ddd}, J_{l}=18.4 \mathrm{~Hz}, J_{2}=9.0 \mathrm{~Hz}, J_{3}=1.1 \mathrm{~Hz}, 1 \mathrm{H}\right), 2.31\left(\mathrm{dd}, J_{l}=18.4\right.$ $\left.\mathrm{Hz}, J_{2}=3.9 \mathrm{~Hz}, 1 \mathrm{H}\right), 2.29-2.20(\mathrm{~m}, 2 \mathrm{H}), 2.24(\mathrm{t}, J=2.5 \mathrm{~Hz}, 1 \mathrm{H}), 2.11(\mathrm{~m}, 1 \mathrm{H}), 2.02(\mathrm{~m}$, $1 \mathrm{H}), 1.92(\mathrm{~m}, 1 \mathrm{H}), 1.76(\mathrm{~m}, 1 \mathrm{H}) \mathrm{ppm} ;{ }^{13} \mathrm{C} \mathrm{NMR}\left(125 \mathrm{MHz}, \mathrm{CDCl}_{3}\right): 200.6,171.1$, 153.4, 125.1, 79.4, 77.1, 71.6, 42.8, 41.1, 32.9, 31.7, 30.4, 23.2 ppm; HRMS (Orbitrap ESI): calcd for $\mathrm{C}_{13} \mathrm{H}_{16} \mathrm{NO}_{2}: 218.1176[\mathrm{M}+\mathrm{H}]^{+}$; found 218.1177.

Representative NOE correlations

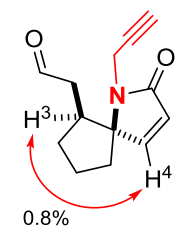

\section{6-(2-oxopropyl)-1-(prop-2-yn-1-yl)-1-azaspiro[4.4]non-3-en-2-one (3e)}

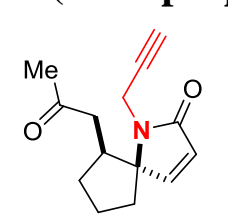

The product was prepared according to the general experimental procedure described above as a $7 / 1$ mixture of separable diastereoisomers. The crude product was purified by flash column chromatography (silica gel, petroleum ether : EtOAc $=1: 1$ ) to furnish $3 \mathbf{e}$ as a brown oil (yield $=26.8 \mathrm{mg}, 58 \%$ ).

${ }^{1} \mathrm{H}$ NMR (500 MHz, $\left.\mathrm{CDCl}_{3}\right): 6.82(\mathrm{~d}, J=5.9 \mathrm{~Hz}, 1 \mathrm{H}), 6.06(\mathrm{~d}, J=5.9 \mathrm{~Hz}, 1 \mathrm{H}), 4.18$ $\left(\mathrm{dd}, J_{l}=17.9 \mathrm{~Hz}, J_{2}=2.5 \mathrm{~Hz}, 1 \mathrm{H}\right), 4.09\left(\mathrm{dd}, J_{l}=17.9 \mathrm{~Hz}, J_{2}=2.5 \mathrm{~Hz}, 1 \mathrm{H}\right), 2.62(\mathrm{~m}, 1 \mathrm{H})$, $2.44\left(\mathrm{dd}, J_{l}=18.1 \mathrm{~Hz}, J_{2}=8.9 \mathrm{~Hz}, 1 \mathrm{H}\right), 2.26-2.18(\mathrm{~m}, 3 \mathrm{H}), 2.24(\mathrm{t}, J=2.5 \mathrm{~Hz}, 1 \mathrm{H}), 2.09$ $(\mathrm{m}, 1 \mathrm{H}), 2.07(\mathrm{~s}, 3 \mathrm{H}), 2.00(\mathrm{~m}, 1 \mathrm{H}), 1.90(\mathrm{~m}, 1 \mathrm{H}), 1.69(\mathrm{~m}, 1 \mathrm{H}) \mathrm{ppm} ;{ }^{13} \mathrm{C} \mathrm{NMR}(125$ $\mathrm{MHz}, \mathrm{CDCl}_{3}$ ): 207.3, 171.2, 153.7, 124.8, 79.5, 77.2, 71.4, 42.3, 41.9, 32.9, 31.7, 30.5, 30.4, 23.1 ppm; HRMS (Orbitrap ESI): calcd for $\mathrm{C}_{14} \mathrm{H}_{18} \mathrm{NO}_{2}: 232.1332[\mathrm{M}+\mathrm{H}]^{+}$; found 232.1333 . 


\section{1-benzyl-6-(2-oxo-2-phenylethyl)-1-azaspiro[4.4]non-3-en-2-one (3f)}

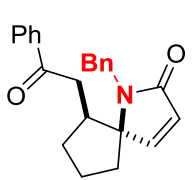

The product was prepared according to the general experimental procedure described above as a 9/1 mixture of separable diastereoisomers. The crude product was purified by flash column chromatography (silica gel, petroleum ether : EtOAc $=2: 1$ ) to furnish $\mathbf{3 f}$ as a brown oil (yield $=42.8 \mathrm{mg}, 62 \%$ ).

${ }^{1} \mathrm{H}$ NMR $\left(500 \mathrm{MHz}, \mathrm{CDCl}_{3}\right): 7.66(\mathrm{~d}, J=7.4 \mathrm{~Hz}, 2 \mathrm{H}), 7.53(\mathrm{t}, J=7.4 \mathrm{~Hz}, 1 \mathrm{H}), 7.40$ $7.33(\mathrm{~m}, 6 \mathrm{H}), 7.27(\mathrm{~m}, 1 \mathrm{H}), 6.90(\mathrm{~d}, J=5.8 \mathrm{~Hz}, 1 \mathrm{H}), 6.17(\mathrm{~d}, J=5.8 \mathrm{~Hz}, 1 \mathrm{H}), 4.69$ (d, $J=15.8 \mathrm{~Hz}, 1 \mathrm{H}), 4.56(\mathrm{~d}, J=15.8 \mathrm{~Hz}, 1 \mathrm{H}), 2.77(\mathrm{~m}, 1 \mathrm{H}), 2.53-2.43(\mathrm{~m}, 2 \mathrm{H}), 2.17(\mathrm{~m}$, $2 \mathrm{H}), 1.93(\mathrm{~m}, 1 \mathrm{H}), 1.87-1.77(\mathrm{~m}, 2 \mathrm{H}), 1.54(\mathrm{~m}, 1 \mathrm{H}) \mathrm{ppm} ;{ }^{13} \mathrm{C} \mathrm{NMR}(125 \mathrm{MHz}$, $\mathrm{CDCl}_{3}$ ): 198.8, 172.6, 153.8, 138.7, 136.6, 133.2, 128.6 (2C), 128.5 (2C), 127.9 (2C), 127.8 (2C), 127.3, 125.0, 77.8, 45.0, 42.7, 37.0, 33.3, 32.1, 23.2 ppm; HRMS (Orbitrap ESI): calcd for $\mathrm{C}_{23} \mathrm{H}_{24} \mathrm{NO}_{2}: 346.1802[\mathrm{M}+\mathrm{H}]^{+}$; found 346.1804 .

\section{1-allyl-6-(2-oxo-2-phenylethyl)-1-azaspiro[4.4]non-3-en-2-one (3g)}

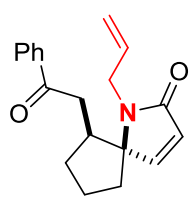

The product was prepared according to the general experimental procedure described above as a 9/1 mixture of separable diastereoisomers. The crude product was purified by flash column chromatography (silica gel, petroleum ether : EtOAc $=3: 1$ ) to furnish $\mathbf{3 g}$ as a brown oil (yield $=36.6 \mathrm{mg}, 62 \%$ ).

${ }^{1} \mathrm{H} \mathrm{NMR}\left(500 \mathrm{MHz}, \mathrm{CDCl}_{3}\right): 7.86(\mathrm{~m}, 2 \mathrm{H}), 7.54(\mathrm{~m}, 1 \mathrm{H}), 7.44(\mathrm{~m}, 2 \mathrm{H}), 6.84(\mathrm{~d}, J=5.8$ $\mathrm{Hz}, 1 \mathrm{H}), 6.07(\mathrm{~d}, J=5.8 \mathrm{~Hz}, 1 \mathrm{H}), 5.97\left(\mathrm{ddt}, J_{l}=17.3 \mathrm{~Hz}, J_{2}=10.3 \mathrm{~Hz}, J_{3}=5.7 \mathrm{~Hz}, 1 \mathrm{H}\right.$ ), $5.19(\mathrm{~m}, 2 \mathrm{H}), 4.19$ (ddt, $\left.J_{l}=16.0 \mathrm{~Hz}, J_{2}=5.8 \mathrm{~Hz}, J_{3}=1.5 \mathrm{~Hz}, 1 \mathrm{H}\right), 3.89$ (ddt, $J_{l}=16.0$ $\left.\mathrm{Hz}, J_{2}=5.8 \mathrm{~Hz}, J_{3}=1.5 \mathrm{~Hz}, 1 \mathrm{H}\right), 2.83-2.75(\mathrm{~m}, 2 \mathrm{H}), 2.68(\mathrm{~m}, 1 \mathrm{H}), 2.30(\mathrm{~m}, 1 \mathrm{H}), 2.21$ $(\mathrm{m}, 1 \mathrm{H}), 2.05-1.94(\mathrm{~m}, 2 \mathrm{H}), 1.88(\mathrm{~m}, 1 \mathrm{H}), 1.61(\mathrm{~m}, 1 \mathrm{H}) \mathrm{ppm} ;{ }^{13} \mathrm{C}$ NMR $(125 \mathrm{MHz}$, $\mathrm{CDCl}_{3}$ ): 198.7, 171.6, 153.3, 136.7, 134.4, 133.2, 128.6 (2C), 127.9 (2C), 125.1, 116.6, 77.3, 44.5, 42.7, 37.0, 33.3, 32.3, 23.3 ppm; HRMS (Orbitrap ESI): calcd for $\mathrm{C}_{19} \mathrm{H}_{22} \mathrm{NO}_{2}: 296.1645[\mathrm{M}+\mathrm{H}]^{+}$; found 296.1647.

\section{1-allyl-6-(2-oxopropyl)-1-azaspiro[4.4]non-3-en-2-one (3h)}

The product was prepared according to the general experimental

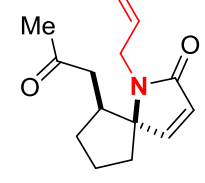
procedure described above as a 7.5/1 mixture of separable diastereoisomers. The crude product was purified by flash column chromatography (silica gel, petroleum ether : EtOAc $=3: 1$ ) to furnish $\mathbf{3 h}$ as a brown oil (yield $=31.2 \mathrm{mg}, 67 \%$ ).

${ }^{1} \mathrm{H}$ NMR (500 MHz, $\mathrm{CDCl}_{3}$ ): $6.77(\mathrm{~d}, J=5.8 \mathrm{~Hz}, 1 \mathrm{H}), 6.04(\mathrm{~d}, J=5.8 \mathrm{~Hz}, 1 \mathrm{H}), 5.89$ (ddt, $\left.J_{1}=17.3 \mathrm{~Hz}, J_{2}=10.3 \mathrm{~Hz}, J_{3}=5.7 \mathrm{~Hz}, 1 \mathrm{H}\right), 5.15(\mathrm{~m}, 2 \mathrm{H}), 4.16$ (ddt, $J_{1}=16.0 \mathrm{~Hz}$, $\left.J_{2}=5.6 \mathrm{~Hz}, J_{3}=1.6 \mathrm{~Hz}, 1 \mathrm{H}\right), 3.76\left(\mathrm{ddt}, J_{l}=16.0 \mathrm{~Hz}, J_{2}=5.9 \mathrm{~Hz}, J_{3}=1.4 \mathrm{~Hz}, 1 \mathrm{H}\right), 2.60$ (m, 1H), 2.25-2.12 (m, 4H), $2.07(\mathrm{~s}, 3 \mathrm{H}), 2.00-1.89(\mathrm{~m}, 2 \mathrm{H}), 1.83(\mathrm{~m}, 1 \mathrm{H}), 1.52(\mathrm{~m}$, 1H) ppm; ${ }^{13} \mathrm{C}$ NMR $\left(125 \mathrm{MHz}, \mathrm{CDCl}_{3}\right): 207.2$ 171.5, 153.4, 134.3, 124.9, 116.5, 77.1, 44.4, 42.2, 42.0, 33.2, 32.2, 30.4, 23.2 ppm; HRMS (Orbitrap ESI): calcd for $\mathrm{C}_{14} \mathrm{H}_{20} \mathrm{NO}_{2}: 234.1488[\mathrm{M}+\mathrm{H}]^{+}$; found 234.1489. 


\section{2-(1-benzyl-2-oxo-1-azaspiro[4.5]dec-3-en-6-yl)acetaldehyde (4a)}
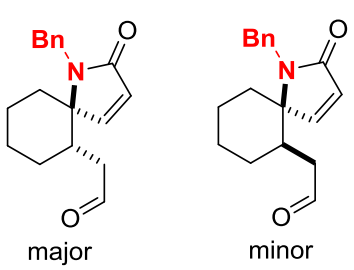

The product was prepared according to the general experimental procedure described above as a 3.5/1 mixture of inseparable diastereoisomers. The crude product was purified by flash column chromatography (silica gel, petroleum ether : $55 \%$ for both isomers).

${ }^{1} \mathrm{H}$ NMR (500 MHz, $\mathrm{CDCl}_{3}$ ): 9.60 (d, $J=1.1 \mathrm{~Hz}, 1 \mathrm{H}$ for minor), $9.03(\mathrm{~d}, J=2.3 \mathrm{~Hz}, 1 \mathrm{H}$ for major), $7.36(\mathrm{~d}, J=7.1 \mathrm{~Hz}, 2 \mathrm{H}$ for major), 7.31-7.19 (m, $4 \mathrm{H}$ for major plus $5 \mathrm{H}$ for minor), 6.78 (d, $J=5.9 \mathrm{~Hz}, 1 \mathrm{H}$ for minor), 6.28 (d, $J=6.2 \mathrm{~Hz}, 1 \mathrm{H}$ for major), 6.17 (d, $J=5.9 \mathrm{~Hz}, 1 \mathrm{H}$ for minor), 5.03 (d, $J=16.3 \mathrm{~Hz}, 1 \mathrm{H}$ for minor), 4.66 (d, $J=15.4 \mathrm{~Hz}, 1 \mathrm{H}$ for major plus $1 \mathrm{H}$ for minor), 4.38 (d, $J=15.4 \mathrm{~Hz}, 1 \mathrm{H}$ for major), 2.41 (m, $1 \mathrm{H}$ for minor), 2.24 (m, $1 \mathrm{H}$ for major plus $1 \mathrm{H}$ for minor), 2.05 (ddd, $J_{l}=18.2 \mathrm{~Hz}, J_{2}=8.8 \mathrm{~Hz}$, $J_{3}=1.6 \mathrm{~Hz}, 1 \mathrm{H}$ for minor), $1.92\left(\mathrm{dd}, J_{l}=17.5 \mathrm{~Hz}, J_{2}=2.1 \mathrm{~Hz}, 1 \mathrm{H}\right.$ for major $), 1.86(\mathrm{~m}$, $1 \mathrm{H}$ for major), $1.83-1.75$ (m, $3 \mathrm{H}$ for major plus $2 \mathrm{H}$ for minor), 1.67 (ddd, $J_{l}=17.5 \mathrm{~Hz}$, $J_{2}=10.2 \mathrm{~Hz}, J_{3}=2.4 \mathrm{~Hz}, 1 \mathrm{H}$ for major), 1.67 (m, $1 \mathrm{H}$ for minor), $1.48(\mathrm{~m}, 1 \mathrm{H}$ for major plus $2 \mathrm{H}$ for minor), $1.40-1.29$ (m, $2 \mathrm{H}$ for major plus $2 \mathrm{H}$ for minor), 1.17 (m, $1 \mathrm{H}$ for major plus $1 \mathrm{H}$ for minor) ppm; ${ }^{13} \mathrm{C} \mathrm{NMR}\left(125 \mathrm{MHz}, \mathrm{CDCl}_{3}\right.$ ): 200.6 (minor), 200.5 (major), 172.7 (minor), 170.7 (major), 155.6 (minor), 148.8 (major), 138.1 (major), 138.0 (minor), 128.5 (2C for major), 128.5 (2C for minor), 128.3 (2C for major), 127.5 (2C for minor), 127.4 (major), 127.0 (minor), 126.7 (major), 125.1 (minor), 70.9 (major), 68.9 (minor), 46.0 (minor), 44.2 (minor), 44.1 (major), 42.3 (major), 35.6 (major), 35.3 (major), 35.1 (minor), 33.7 (minor), 30.4 (major), 28.4 (minor), 25.4 (major), 24.4 (minor), 24.0 (major), 21.5 (minor) ppm; HRMS (Orbitrap ESI): calcd for $\mathrm{C}_{18} \mathrm{H}_{22} \mathrm{NO}_{2}$ : 284.1645 [M+H] ${ }^{+}$; found 284.1647.

Representative NOE correlations for minor diastereoisomer

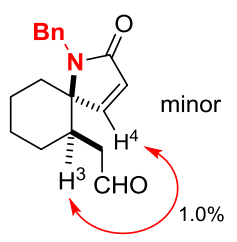

\section{2-(1-allyl-2-oxo-1-azaspiro[4.5]dec-3-en-6-yl)acetaldehyde (4b)}
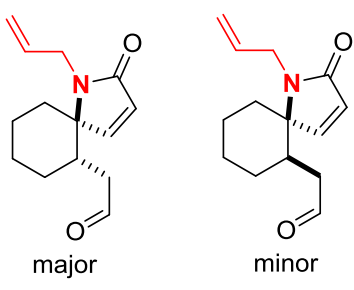

The product was prepared according to the general experimental procedure described above as a 3.5/1 mixture of inseparable diastereoisomers. The crude product was purified by flash column chromatography (silica gel, petroleum ether : EtOAc $=2: 1$ ) to furnish $\mathbf{4 b}$ as a brown oil (yield $=26.1 \mathrm{mg}$, $56 \%$ for both isomers).

${ }^{1} \mathrm{H}$ NMR (500 MHz, $\mathrm{CDCl}_{3}$ ): 9.65 (t, $J=0.7 \mathrm{~Hz}, 1 \mathrm{H}$ for minor), $9.61(\mathrm{~d}, J=1.7 \mathrm{~Hz}, 1 \mathrm{H}$ for major), 7.25 (d, $J=6.0 \mathrm{~Hz}, 1 \mathrm{H}$ for major), 6.72 (d, $J=5.9 \mathrm{~Hz}, 1 \mathrm{H}$ for minor), 6.22 (d, $J=6.0 \mathrm{~Hz}, 1 \mathrm{H}$ for major), 6.07 (d, $J=5.9 \mathrm{~Hz}, 1 \mathrm{H}$ for minor), 5.90-5.77 (m, $1 \mathrm{H}$ for major plus $1 \mathrm{H}$ for minor), 5.21 (dd, $J_{l}=17.2 \mathrm{~Hz}, J_{2}=1.2 \mathrm{~Hz}, 1 \mathrm{H}$ for major), $5.16(\mathrm{~m}$, $2 \mathrm{H}$ for minor), 5.10 (d, $J=10.1 \mathrm{~Hz}, 1 \mathrm{H}$ for major), 4.36 (dd, $J_{l}=16.4 \mathrm{~Hz}, J_{2}=5.4 \mathrm{~Hz}$, 
$1 \mathrm{H}$ for minor), $4.12\left(\mathrm{dd}, J_{l}=16.4 \mathrm{~Hz}, J_{2}=5.2 \mathrm{~Hz}, 1 \mathrm{H}\right.$ for minor), 3.92 (dd, $J_{l}=15.8 \mathrm{~Hz}$, $J_{2}=6.4 \mathrm{~Hz}, 1 \mathrm{H}$ for major), $3.87\left(\mathrm{dd}, J_{1}=15.8 \mathrm{~Hz}, J_{2}=6.0 \mathrm{~Hz}, 1 \mathrm{H}\right.$ for major), $2.43(\mathrm{~m}$, $1 \mathrm{H}$ for major plus $1 \mathrm{H}$ for minor), $2.23\left(\mathrm{dd}, J_{l}=18.1 \mathrm{~Hz}, J_{2}=3.8 \mathrm{~Hz}, 1 \mathrm{H}\right.$ for minor), $2.16(\mathrm{~m}, 1 \mathrm{H}$ for major plus $1 \mathrm{H}$ for minor), $1.96-1.71(\mathrm{~m}, 5 \mathrm{H}$ for major plus $5 \mathrm{H}$ for minor), $1.55-1.35$ (m, $3 \mathrm{H}$ for major plus $2 \mathrm{H}$ for minor), 1.24 (m, $1 \mathrm{H}$ for major plus $1 \mathrm{H}$ for minor) $\mathrm{ppm} ;{ }^{13} \mathrm{C} \mathrm{NMR}\left(125 \mathrm{MHz}, \mathrm{CDCl}_{3}\right.$ ): 200.7 (minor), 200.6 (major), 171.9 (minor), 170.3 (major), 155.1 (minor), 148.6 (major), 133.9 (minor), 133.7 (major), 127.7 (major), 125.4 (minor), 117.4 (major), 116.4 (minor), 70.5 (major), 68.4 (minor), 45.4 (minor), 44.3 (minor), 44.2 (major), 41.6 (major), 35.4 (major), 35.2 (major), 33.7 (minor), 30.5 (major), 29.6 (minor), 28.3 (minor), 25.4 (major), 24.5 (minor), 24.1 (major), 21.6 (minor) ppm; HRMS (Orbitrap ESI): calcd for $\mathrm{C}_{14} \mathrm{H}_{20} \mathrm{NO}_{2}$ : $234.1488[\mathrm{M}+\mathrm{H}]^{+}$; found 234.1489.

\section{6-(2-oxopropyl)-1-(prop-2-yn-1-yl)-1-azaspiro[4.5]dec-3-en-2-one (4c)}

The product was prepared according to the general experimental
procedure described above as a $4 / 1$ mixture of separable
diastereoisomers. The crude product was purified by flash column
chromatography (silica gel, petroleum ether : EtOAc = 1.5:1) to furnish
$\mathbf{4 c}$ as a brown oil (yield $=24.5 \mathrm{mg}, 50 \%$ for major isomer). For major isomer: ${ }^{1} \mathrm{H}$ NMR $\left(500 \mathrm{MHz}, \mathrm{CDCl}_{3}\right): 7.29(\mathrm{~d}, J=6.2 \mathrm{~Hz}, 1 \mathrm{H}), 6.23$ (d, $J=6.2 \mathrm{~Hz}, 1 \mathrm{H}), 4.18\left(\mathrm{dd}, J_{l}=17.9 \mathrm{~Hz}, J_{2}=2.5 \mathrm{~Hz}, 1 \mathrm{H}\right), 3.96\left(\mathrm{dd}, J_{l}=17.9 \mathrm{~Hz}, J_{2}=2.5\right.$ $\mathrm{Hz}, 1 \mathrm{H}), 2.52(\mathrm{~m}, 1 \mathrm{H}), 2.24\left(\mathrm{dd}, J_{1}=15.4 \mathrm{~Hz}, J_{2}=2.6 \mathrm{~Hz}, 1 \mathrm{H}\right), 2.06(\mathrm{t}, J=2.5 \mathrm{~Hz}, 1 \mathrm{H})$, $2.03(\mathrm{~m}, 1 \mathrm{H}), 2.02(\mathrm{~s}, 3 \mathrm{H}), 1.94(\mathrm{~m}, 1 \mathrm{H}), 1.88-1.80(\mathrm{~m}, 3 \mathrm{H}), 1.55-1.40(\mathrm{~m}, 3 \mathrm{H}), 1.17$ (m, 1H) ppm; ${ }^{13} \mathrm{C}$ NMR (125 MHz, $\left.\mathrm{CDCl}_{3}\right):$ 206.8, 169.9, 149.6, 127.1, 78.9, 70.6 (2C), 43.7, 36.6, 34.7, 30.6, 30.3, 27.4, 25.4, 24.0 ppm; HRMS (Orbitrap ESI): calcd for $\mathrm{C}_{15} \mathrm{H}_{20} \mathrm{NO}_{2}: 246.1488[\mathrm{M}+\mathrm{H}]^{+}$; found 246.1490 .

Representative NOE correlations for the major isomer

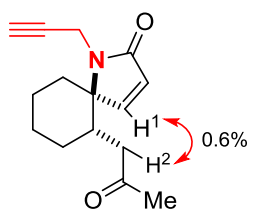

\section{2-(1-(3,4-dimethoxybenzyl)-2-oxo-1-azaspiro[4.5]dec-3-en-6-yl)acetaldehyde (4d)}

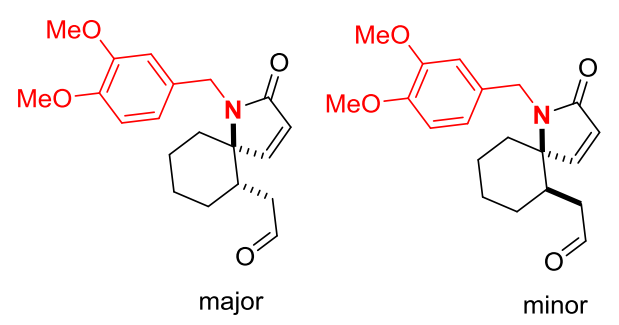
The product was prepared according to the general experimental procedure described above as a 3.2/1 mixture of inseparable diastereoisomers. The crude product was purified by flash column chromatography (silica gel, petroleum ether : EtOAc $=1: 1$ ) to furnish

4d as a brown oil (yield $=39.8 \mathrm{mg}, 58 \%$ for both isomers).

${ }^{1} \mathrm{H}$ NMR (500 MHz, $\mathrm{CDCl}_{3}$ ): 9.60 (d, $J=1.1 \mathrm{~Hz}, 1 \mathrm{H}$ for minor), 9.20 (d, $J=2.0 \mathrm{~Hz}, 1 \mathrm{H}$ for major), 7.25 (d, $J=6.2 \mathrm{~Hz}, 1 \mathrm{H}$ for major), 6.98 (d, $J=1.9 \mathrm{~Hz}, 1 \mathrm{H}$ for major), 6.86 (dd, $J_{1}=8.1 \mathrm{~Hz}, J_{2}=1.9 \mathrm{~Hz}, 1 \mathrm{H}$ for major), 6.78 (m, $3 \mathrm{H}$ for minor), 6.74 (d, $J=8.1 \mathrm{~Hz}$, $1 \mathrm{H}$ for major plus $1 \mathrm{H}$ for minor), 6.28 (d, $J=6.2 \mathrm{~Hz}, 1 \mathrm{H}$ for major), 6.17 (d, $J=5.9 \mathrm{~Hz}$, 
$1 \mathrm{H}$ for minor), 4.94 (d, $J=16.1 \mathrm{~Hz}, 1 \mathrm{H}$ for minor), 4.62 (d, $J=16.1 \mathrm{~Hz}, 1 \mathrm{H}$ for minor), 4.51 (d, $J=15.3 \mathrm{~Hz}, 1 \mathrm{H}$ for major), 4.39 (d, $J=15.3 \mathrm{~Hz}, 1 \mathrm{H}$ for major), 3.85 (s, $3 \mathrm{H}$ for minor), 3.84 (s, $3 \mathrm{H}$ for major plus $3 \mathrm{H}$ for minor), 3.83 (s, $3 \mathrm{H}$ for major), 2.41 (m, $1 \mathrm{H}$ for minor), 2.34 (m, $1 \mathrm{H}$ for major), 2.20 (dd, $J_{l}=18.2 \mathrm{~Hz}, J_{2}=3.4 \mathrm{~Hz}, 1 \mathrm{H}$ for minor), 2.04 (ddd, $J_{l}=18.2 \mathrm{~Hz}, J_{2}=8.7 \mathrm{~Hz}, J_{3}=1.4 \mathrm{~Hz}, 1 \mathrm{H}$ for minor), 1.93 (dd, $J_{l}=17.6 \mathrm{~Hz}$, $J_{2}=2.2 \mathrm{~Hz}, 1 \mathrm{H}$ for major), $1.88-1.66(\mathrm{~m}, 5 \mathrm{H}$ for major plus $4 \mathrm{H}$ for minor), $1.46(\mathrm{~m}, 1 \mathrm{H}$ for major plus $2 \mathrm{H}$ for minor), 1.35 (m, $2 \mathrm{H}$ for major plus $1 \mathrm{H}$ for minor), $1.16(\mathrm{~m}, 1 \mathrm{H}$ for major plus $1 \mathrm{H}$ for minor) ppm; ${ }^{13} \mathrm{C} \mathrm{NMR} \mathrm{(125} \mathrm{MHz,} \mathrm{CDCl}_{3}$ ): 200.6 (minor), 200.5 (major), 172.7 (minor), 170.7 (major), 155.7 (minor), 149.1 (major plus minor), 148.8 (major), 148.4 (major), 148.1 (minor), 130.8 (major plus minor), 127.5 (major), 125.1 (minor), 120.5 (major), 119.1 (minor), 111.7 (major), 111.0 (minor), 110.8 (major), 110.4 (minor), 70.9 (major), 69.0 (minor), 55.9 (2C for major plus 1C for minor), 55.8 (minor), 45.7 (minor), 44.3 (minor), 44.2 (major), 42.2 (major), 35.7 (major), 35.4 (major), 35.1 (minor), 33.8 (minor), 30.4 (major), 28.4 (minor), 25.4 (major), 24.5 (minor), 24.0 (major), 21.7 (minor) ppm; HRMS (Orbitrap ESI): calcd for $\mathrm{C}_{20} \mathrm{H}_{26} \mathrm{NO}_{4}$ : $344.1856[\mathrm{M}+\mathrm{H}]^{+}$; found 344.1859 .

\section{1-allyl-6-(2-oxo-2-phenylethyl)-1-azaspiro[4.5]dec-3-en-2-one (4e)}

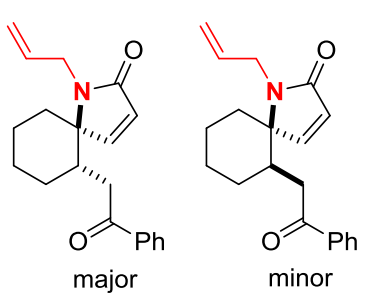

for the minor isomer).

Major isomer: ${ }^{1} \mathrm{H} \mathrm{NMR}\left(500 \mathrm{MHz}, \mathrm{CDCl}_{3}\right): 7.81(\mathrm{~m}, 2 \mathrm{H}), 7.53(\mathrm{t}, J=7.4 \mathrm{~Hz}, 1 \mathrm{H}), 7.41$ $(\mathrm{t}, J=7.7 \mathrm{~Hz}, 2 \mathrm{H}), 7.35(\mathrm{~d}, J=6.2 \mathrm{~Hz}, 1 \mathrm{H}), 6.26(\mathrm{~d}, J=6.2 \mathrm{~Hz}, 1 \mathrm{H}), 5.78$ (ddt, $J_{l}=17.1$ $\left.\mathrm{Hz}, J_{2}=10.2 \mathrm{~Hz}, J_{3}=6.1 \mathrm{~Hz}, 1 \mathrm{H}\right), 5.20\left(\mathrm{dd}, J_{l}=17.1 \mathrm{~Hz}, J_{2}=1.3 \mathrm{~Hz}, 1 \mathrm{H}\right), 5.03(\mathrm{dd}$, $\left.J_{l}=10.2 \mathrm{~Hz}, J_{2}=1.3 \mathrm{~Hz}, 1 \mathrm{H}\right), 3.97\left(\mathrm{dd}, J_{l}=15.8 \mathrm{~Hz}, J_{2}=6.1 \mathrm{~Hz}, 1 \mathrm{H}\right), 3.83\left(\mathrm{dd}, J_{l}=15.8\right.$ $\left.\mathrm{Hz}, J_{2}=6.1 \mathrm{~Hz}, 1 \mathrm{H}\right), 2.62(\mathrm{~m}, 1 \mathrm{H}), 2.55\left(\mathrm{dd}, J_{l}=17.6 \mathrm{~Hz}, J_{2}=2.3 \mathrm{~Hz}, 1 \mathrm{H}\right), 2.45(\mathrm{dd}$, $\left.J_{l}=17.6 \mathrm{~Hz}, J_{2}=9.7 \mathrm{~Hz}, 1 \mathrm{H}\right), 1.97(\mathrm{~m}, 2 \mathrm{H}), 1.83(\mathrm{~m}, 2 \mathrm{H}), 1.56-1.40(\mathrm{~m}, 3 \mathrm{H}), 1.22(\mathrm{qd}$, $\left.J_{l}=12.7 \mathrm{~Hz}, J_{2}=3.4 \mathrm{~Hz}, 1 \mathrm{H}\right) \mathrm{ppm} ;{ }^{13} \mathrm{C} \mathrm{NMR}\left(125 \mathrm{MHz}, \mathrm{CDCl}_{3}\right): 198.4,170.3,149.1$, 136.9, 133.6, 133.1, 128.5 (2C), 127.8 (2C), 127.5, 117.3, 70.9, 41.6, 38.2, 36.8, 35.6, 30.6, 25.5, 24.2 ppm; HRMS (Orbitrap ESI): calcd for $\mathrm{C}_{20} \mathrm{H}_{24} \mathrm{NO}_{2}: 310.1802[\mathrm{M}+\mathrm{H}]^{+}$; found 310.1804 .

Representative NOE correlations

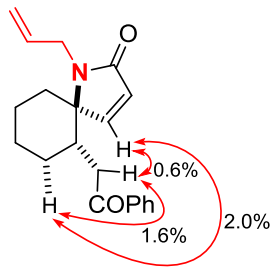

Minor isomer: ${ }^{1} \mathrm{H}$ NMR (500 MHz, $\left.\mathrm{CDCl}_{3}\right)$ : $7.84(\mathrm{~m}, 2 \mathrm{H}), 7.54(\mathrm{~m}, 1 \mathrm{H}), 7.43(\mathrm{t}$, $J=7.7 \mathrm{~Hz}, 2 \mathrm{H}), 6.77(\mathrm{~d}, J=5.9 \mathrm{~Hz}, 1 \mathrm{H}), 6.05$ (d, $J=5.9 \mathrm{~Hz}, 1 \mathrm{H}), 5.94$ (ddt, $J_{l}=17.6 \mathrm{~Hz}$, 
$\left.J_{2}=10.2 \mathrm{~Hz}, J_{3}=5.2 \mathrm{~Hz}, 1 \mathrm{H}\right), 5.20(\mathrm{~m}, 2 \mathrm{H}), 4.45\left(\mathrm{ddt}, J_{l}=16.3 \mathrm{~Hz}, J_{2}=5.2 \mathrm{~Hz}, J_{3}=1.5\right.$ $\mathrm{Hz}, 1 \mathrm{H}), 4.23$ (ddt, $\left.J_{l}=16.3 \mathrm{~Hz}, J_{2}=5.2 \mathrm{~Hz}, J_{3}=1.5 \mathrm{~Hz}, 1 \mathrm{H}\right), 2.67(\mathrm{~m}, 2 \mathrm{H}), 2.59$ (m, 1H), $1.89(\mathrm{~m}, 3 \mathrm{H}), 1.73(\mathrm{~m}, 3 \mathrm{H}), 1.53(\mathrm{~m}, 2 \mathrm{H}) \mathrm{ppm} ;{ }^{13} \mathrm{C} \mathrm{NMR}\left(125 \mathrm{MHz}, \mathrm{CDCl}_{3}\right)$ : 198.8, 172.1, 155.5, 136.9, 134.1, 133.2, 128.6 (2C), 127.9 (2C), 125.2, 116.3, 68.8, 45.5, 38.4, 36.7, 34.0, 28.2, 24.7, 21.7 ppm; HRMS (Orbitrap ESI): calcd for $\mathrm{C}_{20} \mathrm{H}_{24} \mathrm{NO}_{2}: 310.1802[\mathrm{M}+\mathrm{H}]^{+}$; found 310.1803 .

\section{Synthesis of the tricyclic compound 6}

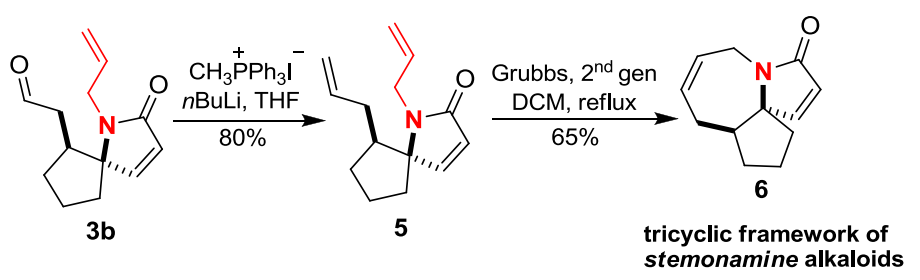

\section{1,6-diallyl-1-azaspiro[4.4]non-3-en-2-one (5)}

To a suspension of methyltriphenylphosphonium iodide $(73.8 \mathrm{mg}, 0.183 \mathrm{mmol})$ in dry THF $(1 \mathrm{~mL})$, at $0{ }^{\circ} \mathrm{C}$ and under argon atmosphere, a solution of $n \mathrm{BuLi}(107 \mu \mathrm{L}, 1.6 \mathrm{M}$ in hexane, $0.171 \mathrm{mmol}$ ) was added slowly. After stirring for $15 \mathrm{~min}$ at the same temperature, a solution of the aldehyde $\mathbf{3 b}(25 \mathrm{mg}, 0.114 \mathrm{mmol})$ in dry THF $(0.8 \mathrm{~mL})$ was added dropwise. The solution was stirred at the same temperature for $5 \mathrm{~min}$ and then allowed to warm to room temperature and further stirred for 30 mins at which point full consumption of the aldehyde was indicated by tlc analysis. The reaction was diluted with EtOAc ( $5 \mathrm{~mL}$ ) and washed with a saturated aqueous solution of $\mathrm{NH}_{4} \mathrm{Cl}(5$ $\mathrm{mL})$ and then with brine $(5 \mathrm{~mL})$. The layers were separated and the organic layer was dried over $\mathrm{Na}_{2} \mathrm{SO}_{4}$, filtered and concentrated in vacuo. The residue was purified by flash column chromatography (silica gel, petroleum ether : EtOAc $=10: 1$ ) to afford compound 5 as a slightly yellow oil. Yield $=19.8 \mathrm{mg}(80 \%)$.

${ }^{1} \mathrm{H}$ NMR $\left(500 \mathrm{MHz}, \mathrm{CDCl}_{3}\right): 6.79(\mathrm{~d}, J=5.8 \mathrm{~Hz}, 1 \mathrm{H}), 6.06(\mathrm{~d}, J=5.8 \mathrm{~Hz}, 1 \mathrm{H}), 5.90(\mathrm{~m}$, $1 \mathrm{H}), 5.68(\mathrm{~m}, 1 \mathrm{H}), 5.14(\mathrm{~m}, 2 \mathrm{H}), 4.97(\mathrm{~m}, 1 \mathrm{H}), 4.93(\mathrm{~m}, 1 \mathrm{H}), 4.26\left(\mathrm{ddt}, J_{l}=16.2 \mathrm{~Hz}\right.$, $\left.J_{2}=5.1 \mathrm{~Hz}, J_{3}=1.6 \mathrm{~Hz}, 1 \mathrm{H}\right), 3.73\left(\mathrm{dd}, J_{l}=16.2 \mathrm{~Hz}, J_{2}=6.0 \mathrm{~Hz}, 1 \mathrm{H}\right), 2.11(\mathrm{~m}, 3 \mathrm{H}), 1.96$ $(\mathrm{m}, 2 \mathrm{H}), 1.87(\mathrm{~m}, 1 \mathrm{H}), 1.78(\mathrm{~m}, 2 \mathrm{H}), 1.60(\mathrm{~m}, 1 \mathrm{H}) \mathrm{ppm} ;{ }^{13} \mathrm{C}$ NMR $(125 \mathrm{MHz}$, $\left.\mathrm{CDCl}_{3}\right): 171.5,153.4,136.9,134.4,124.9,116.1,115.7,76.7,47.4,44.6,33.8,32.8$, 32.0, 23.2 ppm; HRMS (Orbitrap ESI): calcd for $\mathrm{C}_{14} \mathrm{H}_{20} \mathrm{NO}$ : $218.1539[\mathrm{M}+\mathrm{H}]^{+}$; found 218.1540 .

\section{5,8,8a,9,10,11-hexahydro-3H-cyclopenta[b]pyrrolo[1,2-a]azepin-3-one (6)}

A solution containing compound $5(19.8 \mathrm{mg}, 0.0912 \mathrm{mmol})$ and Grubbs catalyst $\left(2^{\text {nd }}\right.$ generation, $3.9 \mathrm{mg}, 0.00456 \mathrm{mmol}, 5 \% \mathrm{~mol})$ in dry DCM $(7 \mathrm{~mL})$ was degassed with argon (balloon) for $10 \mathrm{~min}$. The solution was heated to reflux with an oil bath, under an argon atmosphere, for $2 \mathrm{~h}$ and an additional amount of Grubbs catalyst $\left(2^{\text {nd }}\right.$ generation, $3.9 \mathrm{mg}, 0.00456 \mathrm{mmol}, 5 \% \mathrm{~mol}$ ) was added. The solution was heated to reflux with an oil bath until the full consumption of the starting compound $\mathbf{5}$ was observed ( $2 \mathrm{~h}, 4 \mathrm{~h}$ heating in total). The solvent was removed under reduced pressure and the residue was purified by flash column chromatography (silica gel, petroleum 
ether $:$ EtOAc $=9: 1 \rightarrow 2: 1)$ to afford compound $\mathbf{6}$ as a slightly yellow oil. Yield $=$ $11.2 \mathrm{mg}(65 \%)$.

${ }^{1} \mathrm{H}$ NMR $\left(500 \mathrm{MHz}, \mathrm{CDCl}_{3}\right): 6.87(\mathrm{~d}, J=5.7 \mathrm{~Hz}, 1 \mathrm{H}), 6.04(\mathrm{~d}, J=5.7 \mathrm{~Hz}, 1 \mathrm{H}), 5.52(\mathrm{~m}$, 2H), $4.68(\mathrm{~m}, 1 \mathrm{H}), 3.47(\mathrm{~m}, 1 \mathrm{H}), 2.33(\mathrm{~m}, 1 \mathrm{H}), 2.12(\mathrm{~m}, 1 \mathrm{H}), 2.05-1.92(\mathrm{~m}, 4 \mathrm{H}), 1.89$ $1.76(\mathrm{~m}, 2 \mathrm{H}), 1.61(\mathrm{~m}, 1 \mathrm{H}) \mathrm{ppm} ;{ }^{13} \mathrm{C} \mathrm{NMR}\left(125 \mathrm{MHz}, \mathrm{CDCl}_{3}\right): 170.4,153.6,126.9$, 126.4, 123.6, 75.6, 45.6, 39.8, 32.4, 30.3, 24.9, 24.5 ppm; HRMS (Orbitrap ESI): calcd for $\mathrm{C}_{12} \mathrm{H}_{16} \mathrm{NO}$ : $190.1226[\mathrm{M}+\mathrm{H}]^{+}$; found 190.1228 .

\section{Synthesis of the tricyclic compounds 9 and 10}

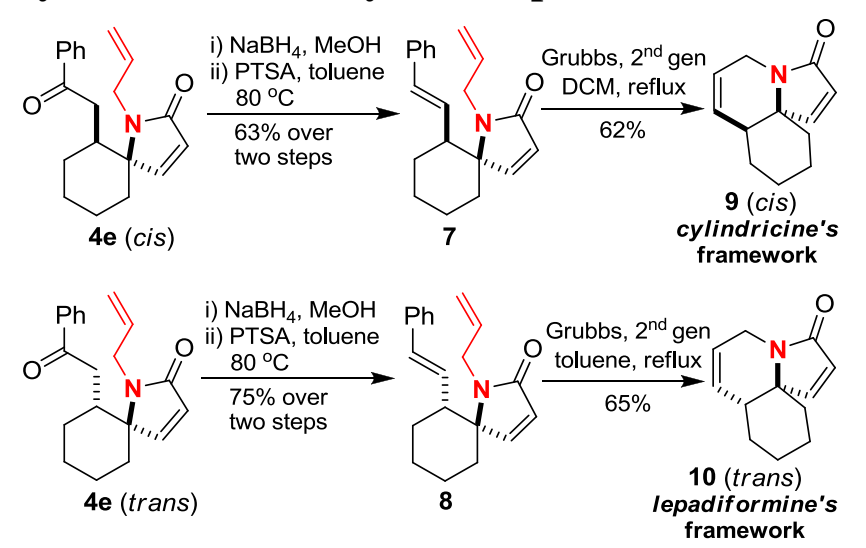

To a solution of sodium borohydride $(7.6 \mathrm{mg}, 0.2 \mathrm{mmol})$ in anhydrous $\mathrm{MeOH}(1 \mathrm{~mL})$ at $0{ }^{\circ} \mathrm{C}$, a solution of the spirocyclic compound $4 \mathbf{e}(61.8 \mathrm{mg}, 0.2 \mathrm{mmol}$ for the cis isomer, or $61.8 \mathrm{mg}, 0.2 \mathrm{mmol}$ for the trans isomer) in dry $\mathrm{MeOH}(0.5 \mathrm{~mL})$ was added slowly. The solution was stirred for $30 \mathrm{~min}$ at the same temperature and then warmed to room temperature and stirred for a further $1 \mathrm{~h}$. After that, completion of the reaction was indicated by TLC analysis and the reaction was quenched with a saturated aqueous solution $\mathrm{NH}_{4} \mathrm{Cl}(0.5 \mathrm{~mL})$ and distilled water was then added $(1.5$ $\mathrm{mL})$. The resulting solution was extracted with EtOAc $(2 \times 4 \mathrm{~mL})$. The combined organic layers were dried over $\mathrm{Na}_{2} \mathrm{SO}_{4}$, filtered and concentrated in vacuo. The corresponding alcohol products were used in the next step without further purification.

For each alcohol, a solution containing the alcohol and PTSA $(22.8 \mathrm{mg}, 0.12 \mathrm{mmol})$ in toluene $(5 \mathrm{~mL})$, was heated at $80{ }^{\circ} \mathrm{C}$ with an oil bath for $12 \mathrm{~h}$, until full consumption of the starting material was indicated by tlc analysis. Then the solution was diluted by EtOAc $(8 \mathrm{~mL})$ and washed by a saturated aqueous solution of $\mathrm{NaHCO}_{3}$ $(5 \mathrm{~mL})$ and brine $(5 \mathrm{~mL})$. The organic layer was dried over $\mathrm{Na}_{2} \mathrm{SO}_{4}$, filtered and concentrated in vacuo. The residue was purified by flash column chromatography (silica gel, petroleum ether : EtOAc $=2: 1$ for both products) to afford compound 7 or $\mathbf{8}$ as slightly yellow oils. Yield $=36.9 \mathrm{mg}(63 \%)$ for 7 and $44.0 \mathrm{mg}(75 \%)$ for $\mathbf{8}$.

\section{(E)-1-allyl-6-styryl-1-azaspiro[4.5]dec-3-en-2-one (7 and 8)}

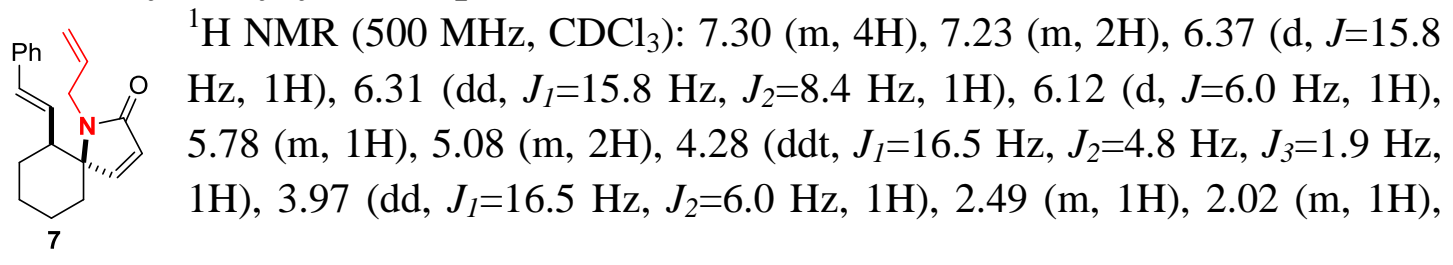


$1.93(\mathrm{~m}, 1 \mathrm{H}), 1.86(\mathrm{~m}, 1 \mathrm{H}), 1.79(\mathrm{~m}, 2 \mathrm{H}), 1.70(\mathrm{~m}, 1 \mathrm{H}), 1.60(\mathrm{~m}, 2 \mathrm{H}) \mathrm{ppm} ;{ }^{13} \mathrm{C} \mathrm{NMR}$ (125 MHz, $\left.\mathrm{CDCl}_{3}\right)$ : 171.4, 153.5, 136.9, 134.3, 132.0, 128.6 (2C), 128.2, 127.4, 126.2 (2C), 125.3, 115.9, 70.1, 46.8, 44.9, 31.7, 30.3, 23.0, 21.9 ppm; HRMS (Orbitrap ESI): calcd for $\mathrm{C}_{20} \mathrm{H}_{24} \mathrm{NO}$ : $294.1852[\mathrm{M}+\mathrm{H}]^{+}$; found 294.1855 .

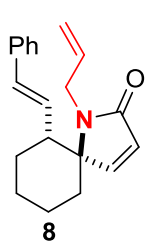

${ }^{1} \mathrm{H}$ NMR $\left(500 \mathrm{MHz}, \mathrm{CDCl}_{3}\right): 7.33(\mathrm{~d}, J=6.2 \mathrm{~Hz}, 1 \mathrm{H}), 7.25-7.16(\mathrm{~m}, 5 \mathrm{H})$, $6.32(\mathrm{~d}, J=15.9 \mathrm{~Hz}, 1 \mathrm{H}), 6.23(\mathrm{~d}, J=6.2 \mathrm{~Hz}, 1 \mathrm{H}), 5.88\left(\mathrm{ddt}, J_{l}=17.0 \mathrm{~Hz}\right.$, $\left.J_{2}=10.3 \mathrm{~Hz}, J_{3}=5.9 \mathrm{~Hz}, 1 \mathrm{H}\right), 5.70\left(\mathrm{dd}, J_{l}=15.9 \mathrm{~Hz}, J_{2}=7.8 \mathrm{~Hz}, 1 \mathrm{H}\right), 5.23$ $(\mathrm{m}, 1 \mathrm{H}), 5.13(\mathrm{~m}, 1 \mathrm{H}), 4.16\left(\mathrm{ddt}, J_{l}=16.0 \mathrm{~Hz}, J_{2}=5.6 \mathrm{~Hz}, J_{3}=1.5 \mathrm{~Hz}, 1 \mathrm{H}\right)$, $3.83\left(\mathrm{dd}, J_{l}=16.0 \mathrm{~Hz}, J_{2}=6.2 \mathrm{~Hz}, 1 \mathrm{H}\right), 2.63(\mathrm{~m}, 1 \mathrm{H}), 1.98-1.86(\mathrm{~m}, 4 \mathrm{H})$, 1.62-1.49 (m, 3H), $1.42(\mathrm{~m}, 1 \mathrm{H}) \mathrm{ppm} ;{ }^{13} \mathrm{C} \mathrm{NMR}\left(125 \mathrm{MHz}, \mathrm{CDCl}_{3}\right)$ : 170.3, 148.6, 137.0, 134.6, 131.6, 128.5, 128.4 (2C), 127.6, 127.3, 126.1 (2C), 116.5, 71.4, 45.2, 41.8, 35.5, 30.6, 25.3, 24.0 ppm; HRMS (Orbitrap ESI): calcd for $\mathrm{C}_{20} \mathrm{H}_{24} \mathrm{NO}$ : $294.1852[\mathrm{M}+\mathrm{H}]^{+}$; found 294.1855 .

A solution containing compound 7 or $8(36.0 \mathrm{mg}, 0.123 \mathrm{mmol}$ for 7; or $43.0 \mathrm{mg}$, $0.146 \mathrm{mmol}$ for 8) and Grubbs catalyst ( $2^{\text {nd }}$ generation, $5.2 \mathrm{mg}, 0.00615 \mathrm{mmol}, 5 \%$ mol for 7; or $3.1 \mathrm{mg}, 0.0037 \mathrm{mmol}, 2.5 \% \mathrm{~mol}$ for 8) in dry DCM (10 mL in case of 7) or dry toluene (12 mL in case of 8) was degassed with argon (balloon) for $10 \mathrm{~min}$. The solution was heated to reflux with an oil bath, under argon atmosphere, for $2 \mathrm{~h}$ and an additional amount of Grubbs catalyst $\left(2^{\text {nd }}\right.$ generation, $5.2 \mathrm{mg}, 0.00615 \mathrm{mmol}$, $5 \% \mathrm{~mol}$ for 7; or $3.1 \mathrm{mg}, 0.0037 \mathrm{mmol}, 2.5 \% \mathrm{~mol}$ for $\mathbf{8}$ ) was added. The solution was heated to reflux with an oil bath, until the full consumption of the starting compound 7 or 8 ( 2 h, 4 h heating in total for both cases). The solvent was removed under reduced pressure and the residue was purified by flash column chromatography (silica gel, petroleum ether : EtOAc $=3: 1$ for product 9, or 1:1 for product 10) to afford compound $\mathbf{9}$ or $\mathbf{1 0}$ as slightly yellow oils. Yield for $\mathbf{9}=14.4 \mathrm{mg}(62 \%)$ and yield for $\mathbf{1 0}=17.9 \mathrm{mg}(65 \%)$.

\section{5,7a,8,9,10,11-hexahydro-3H-pyrrolo[2,1-j]quinolin-3-one (9 and 10)}

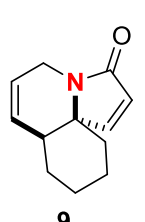

${ }^{1} \mathrm{H}$ NMR $\left(500 \mathrm{MHz}, \mathrm{CDCl}_{3}\right): 7.52(\mathrm{~d}, J=6.0 \mathrm{~Hz}, 1 \mathrm{H}), 6.19(\mathrm{~d}, J=6.0 \mathrm{~Hz}$, $1 \mathrm{H}), 5.84\left(\mathrm{dq}, J_{l}=10.0 \mathrm{~Hz}, J_{2}=2.9 \mathrm{~Hz}, 1 \mathrm{H}\right), 5.54\left(\mathrm{dq}, J_{l}=10.0 \mathrm{~Hz}, J_{2}=2.1\right.$ $\mathrm{Hz}, 1 \mathrm{H}), 4.48\left(\mathrm{dq}, J_{l}=18.9 \mathrm{~Hz}, J_{3}=2.9 \mathrm{~Hz}, 1 \mathrm{H}\right), 3.50(\mathrm{~m}, 1 \mathrm{H}), 2.24$ (brs, $1 \mathrm{H}), 1.84(\mathrm{~m}, 2 \mathrm{H}), 1.74(\mathrm{~m}, 2 \mathrm{H}), 1.59(\mathrm{~m}, 1 \mathrm{H}), 1.44$ (qt, $J_{l}=13.4 \mathrm{~Hz}, J_{2}=3.5$ $\mathrm{Hz}, 1 \mathrm{H}), 1.28\left(\mathrm{qt}, J_{l}=13.4 \mathrm{~Hz}, J_{2}=3.7 \mathrm{~Hz}, 1 \mathrm{H}\right), 1.20(\mathrm{~m}, 1 \mathrm{H}) \mathrm{ppm} ;{ }^{13} \mathrm{C} \mathrm{NMR}$ (125 MHz, $\left.\mathrm{CDCl}_{3}\right): 168.5,151.3,128.6,126.5,124.0,64.0,38.7,37.5,29.6,28.1$, 23.5, 20.5 ppm; HRMS (Orbitrap ESI): calcd for $\mathrm{C}_{12} \mathrm{H}_{16} \mathrm{NO}$ : $190.1226[\mathrm{M}+\mathrm{H}]^{+}$; found 190.1228 .

Representative NOE correlations

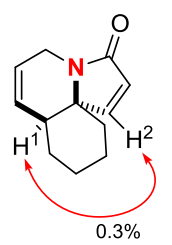


H NMR $\left(500 \mathrm{MHz}, \mathrm{CDCl}_{3}\right): 7.54(\mathrm{~d}, J=5.9 \mathrm{~Hz}, 1 \mathrm{H}), 6.14(\mathrm{~d}, J=5.9 \mathrm{~Hz}$, $1 \mathrm{H}), 6.00(\mathrm{~m}, 1 \mathrm{H}), 5.65\left(\mathrm{dt}, J_{l}=8.6 \mathrm{~Hz}, J_{2}=3.3 \mathrm{~Hz}, 1 \mathrm{H}\right), 4.52\left(\mathrm{dd}, J_{I}=15.0\right.$ $\left.\mathrm{Hz}, J_{2}=6.3 \mathrm{~Hz}, 1 \mathrm{H}\right), 3.36\left(\mathrm{dt}, J_{I}=15.0 \mathrm{~Hz}, J_{2}=3.0 \mathrm{~Hz}, 1 \mathrm{H}\right), 2.40(\mathrm{~m}, 1 \mathrm{H})$, $1.99(\mathrm{~m}, 3 \mathrm{H}), 1.87(\mathrm{~m}, 1 \mathrm{H}), 1.75-1.61(\mathrm{~m}, 2 \mathrm{H}), 1.60-1.47(\mathrm{~m}, 2 \mathrm{H}) \mathrm{ppm} ;{ }^{13} \mathrm{C}$ NMR $\left(125 \mathrm{MHz}, \mathrm{CDCl}_{3}\right): 180.2,154.3,131.5,129.5,129.4,70.7,44.8$, 41.2, 38.5, 27.3, 26.5, $24.1 \mathrm{ppm}$; HRMS (Orbitrap ESI): calcd for $\mathrm{C}_{12} \mathrm{H}_{16} \mathrm{NO}$ : $190.1226[\mathrm{M}+\mathrm{H}]^{+}$; found 190.1228 . 
Part B: Copies of ${ }^{1} \mathrm{H}-\mathrm{NMR},{ }^{13} \mathrm{C}-\mathrm{NMR}, \mathrm{COSY}$, HSQC, HMBC and NOE spectra

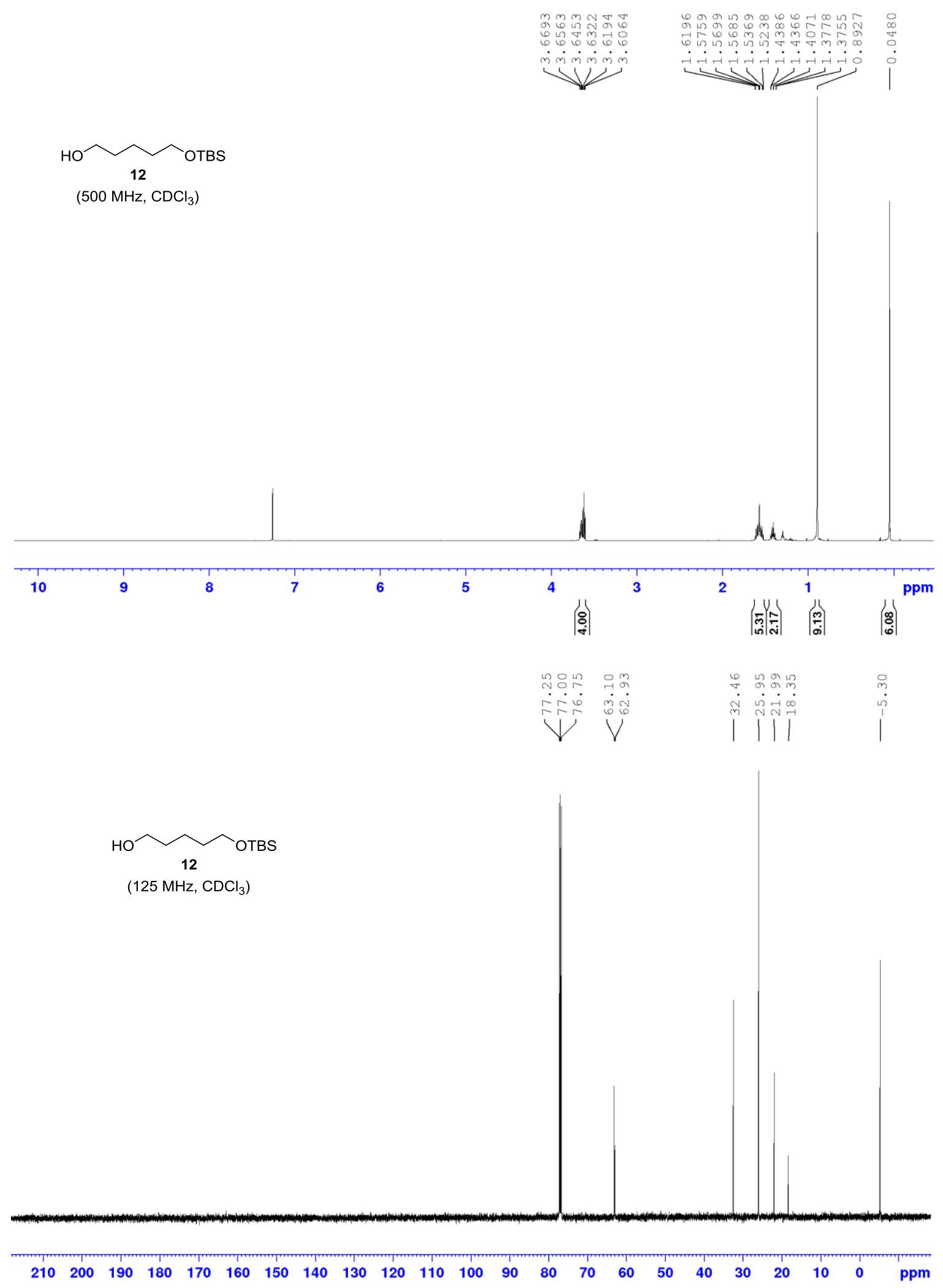




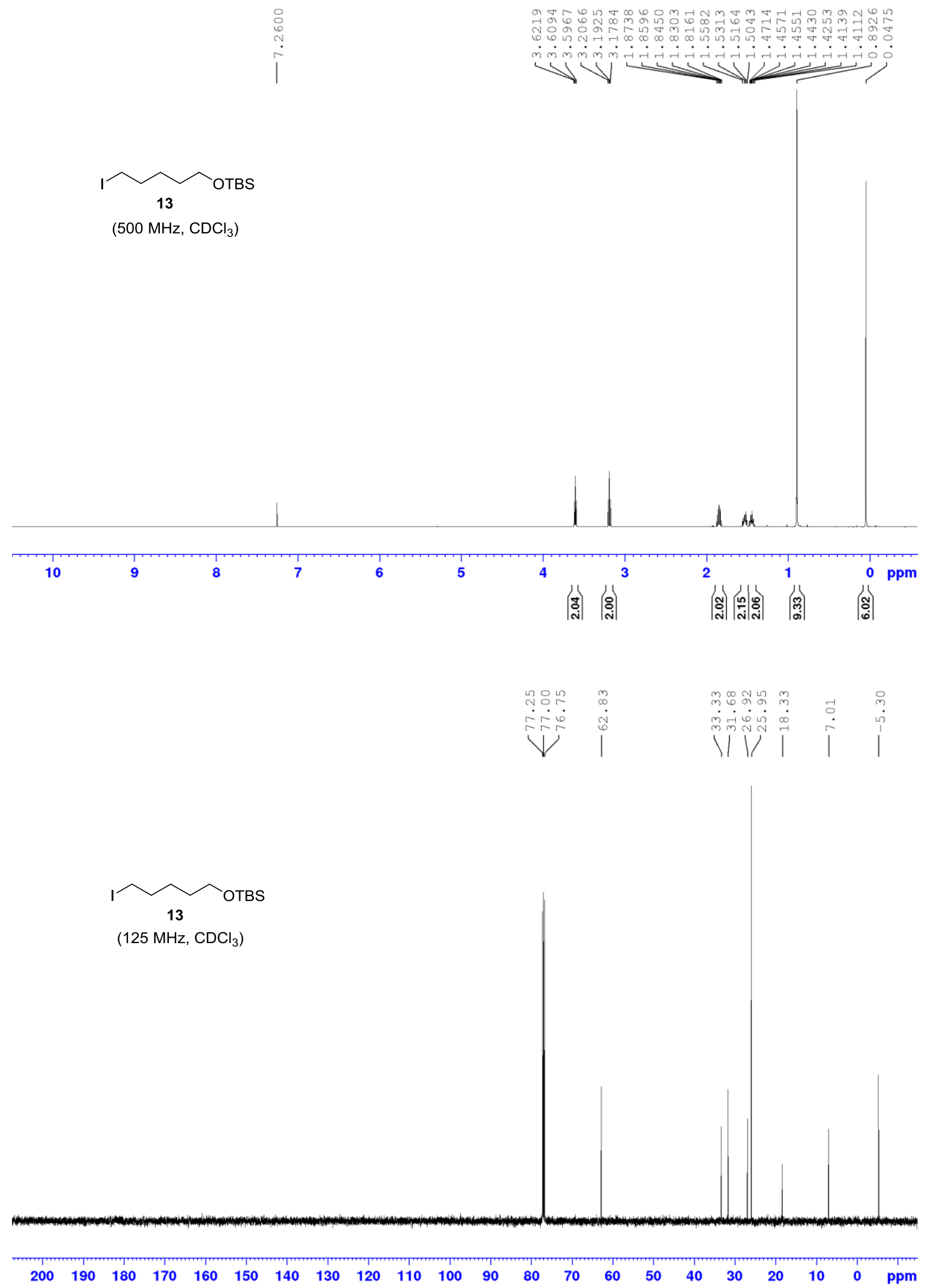



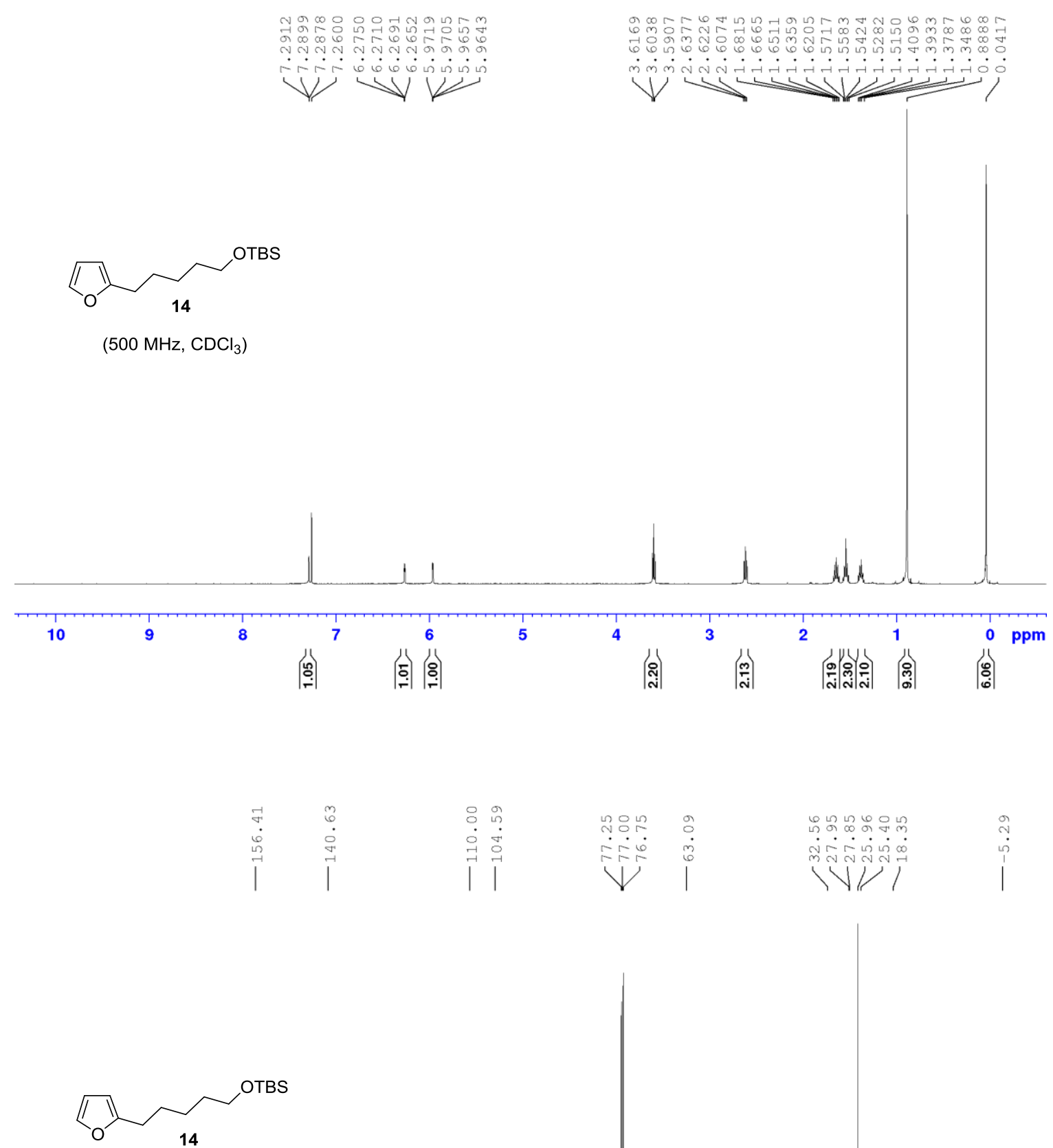

(125 MHz, $\mathrm{CDCl}_{3}$ )

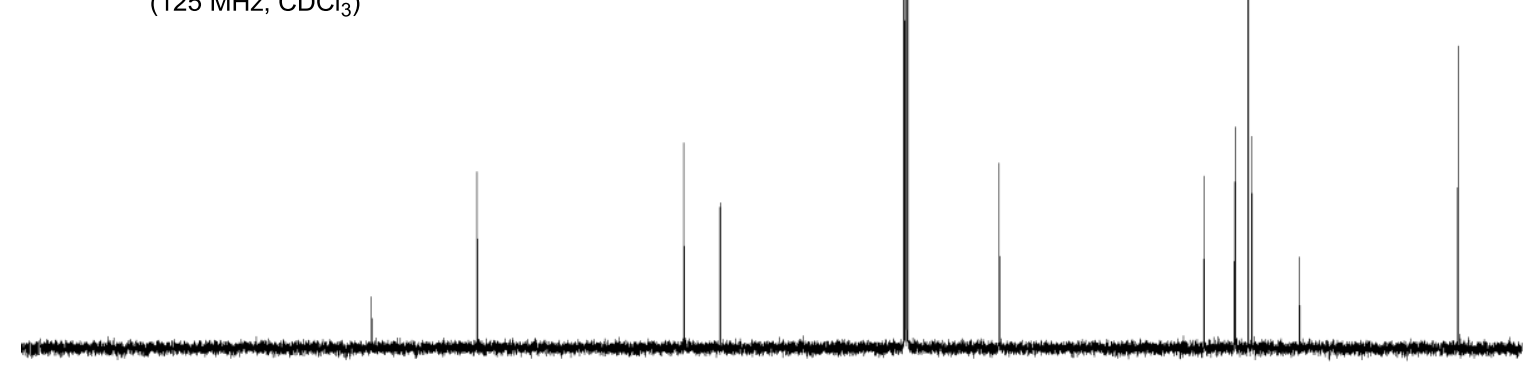

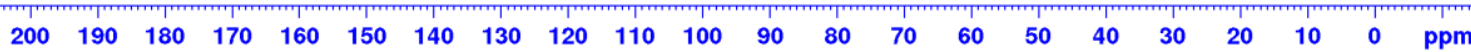



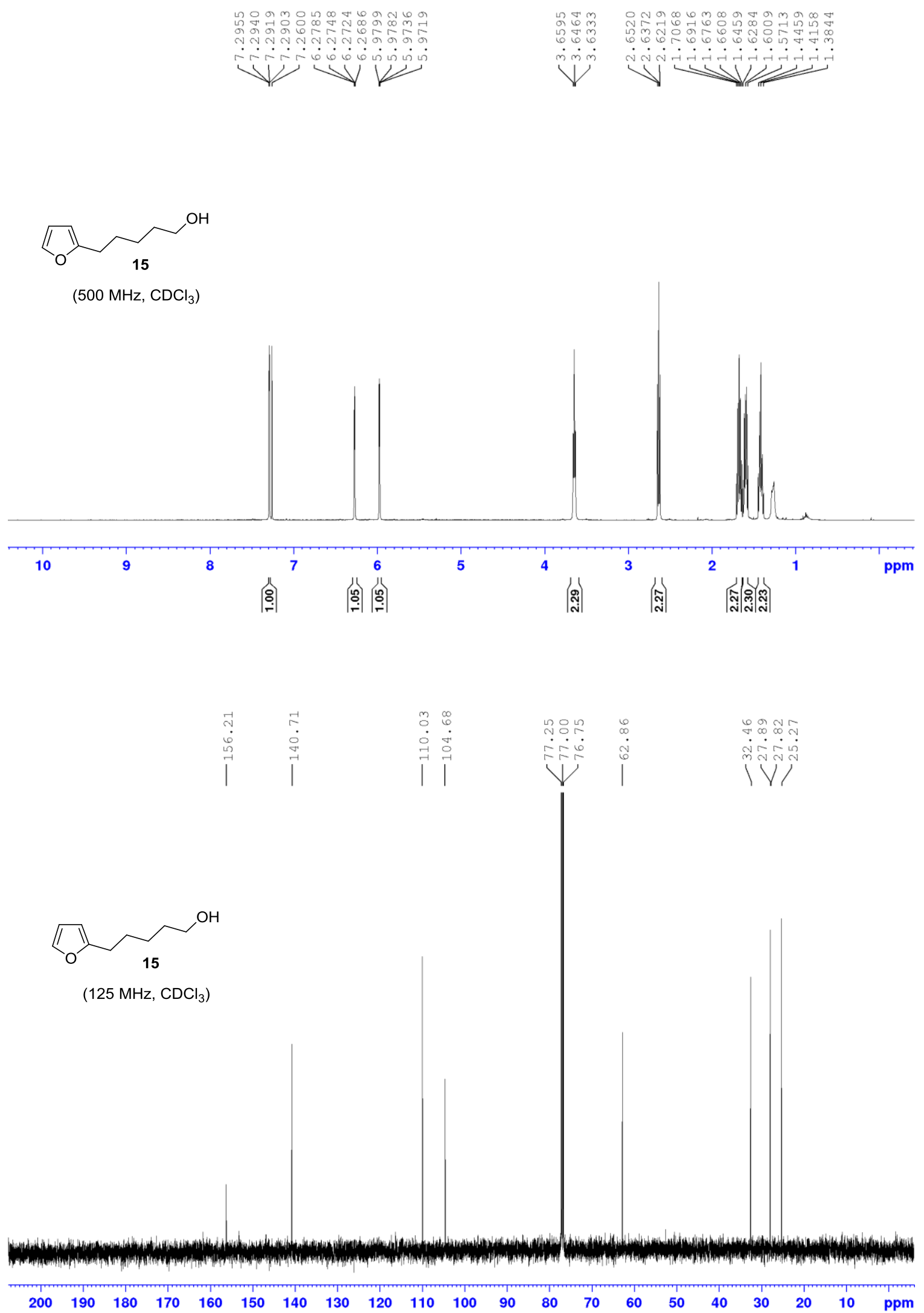


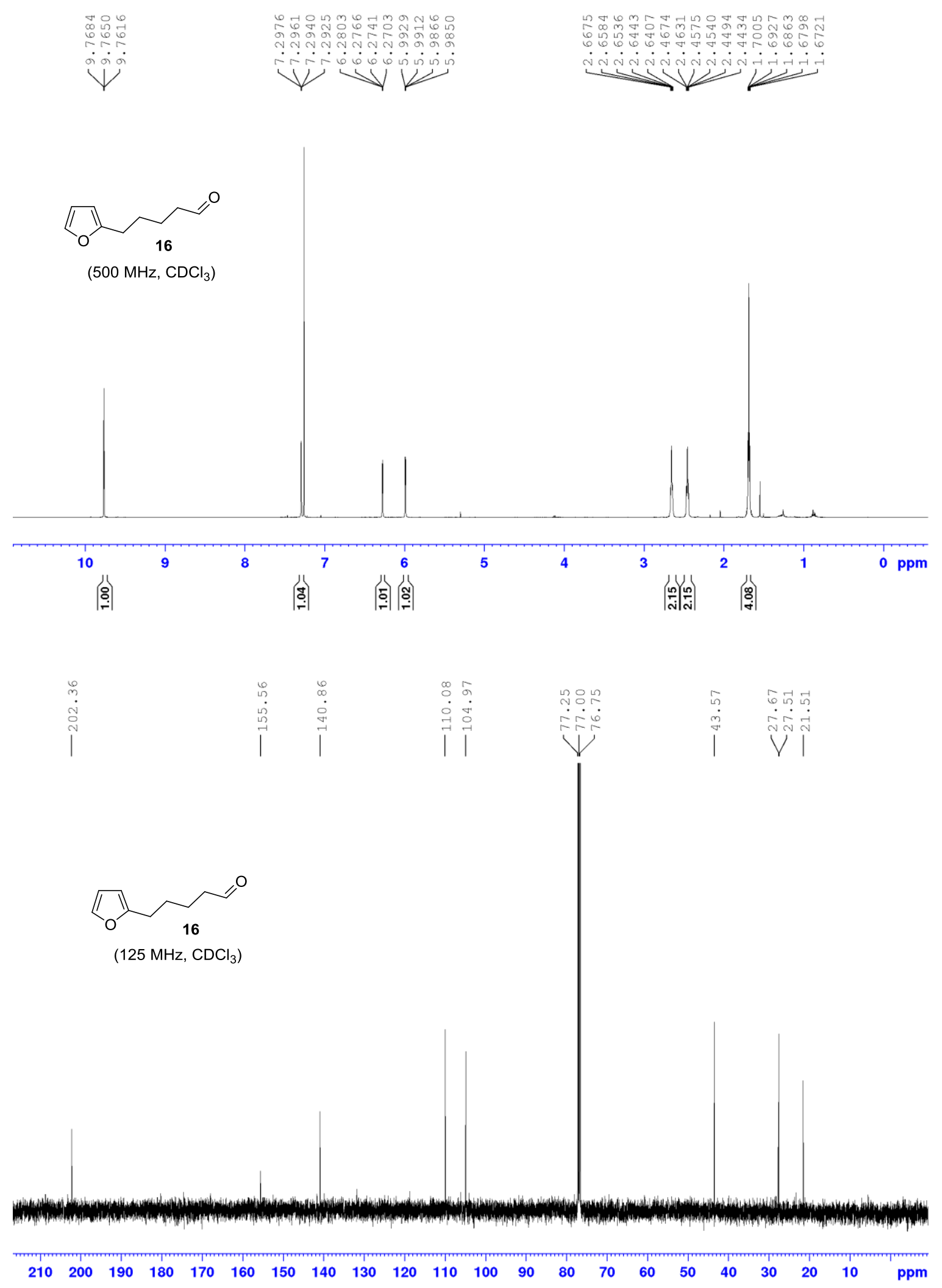




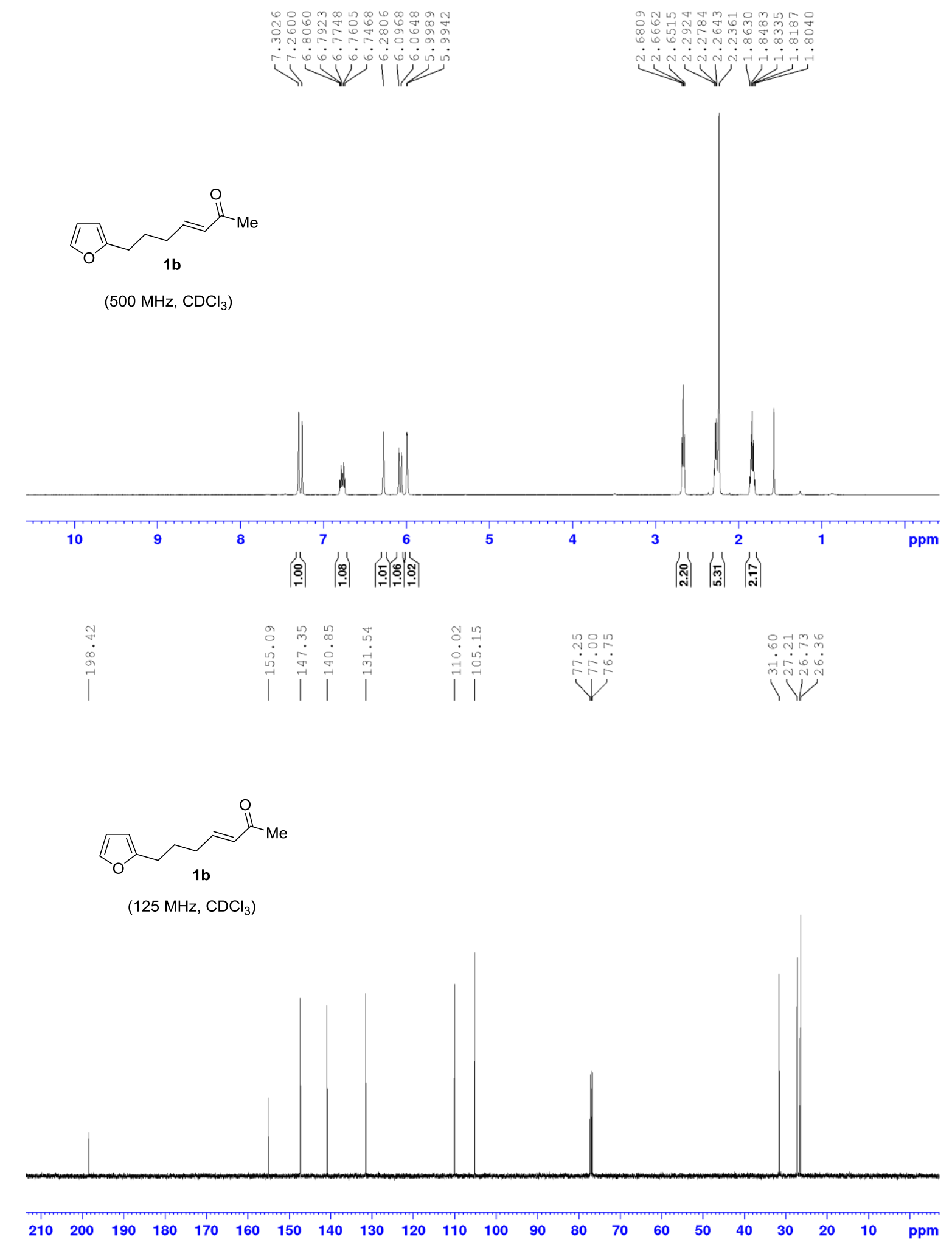




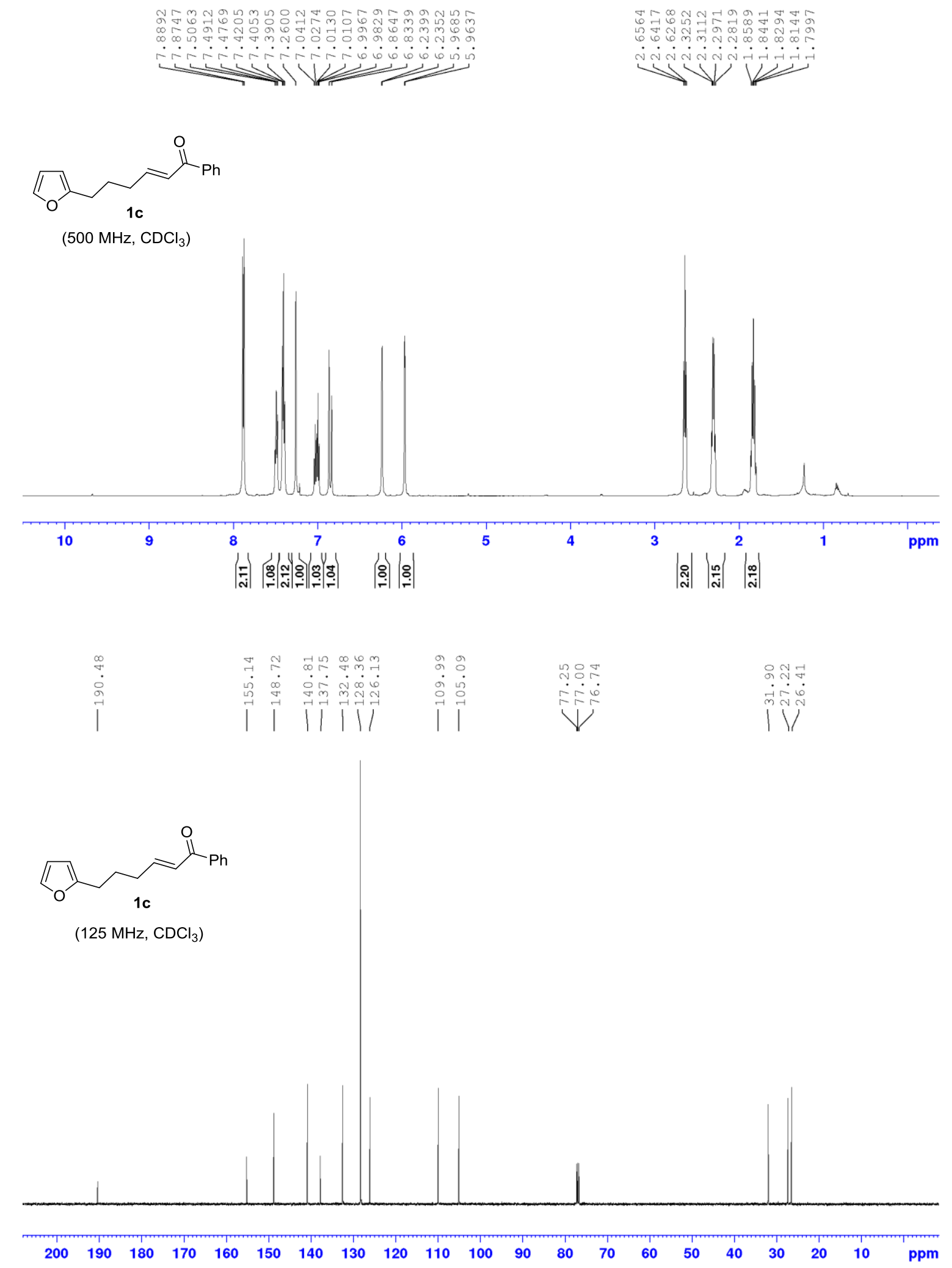



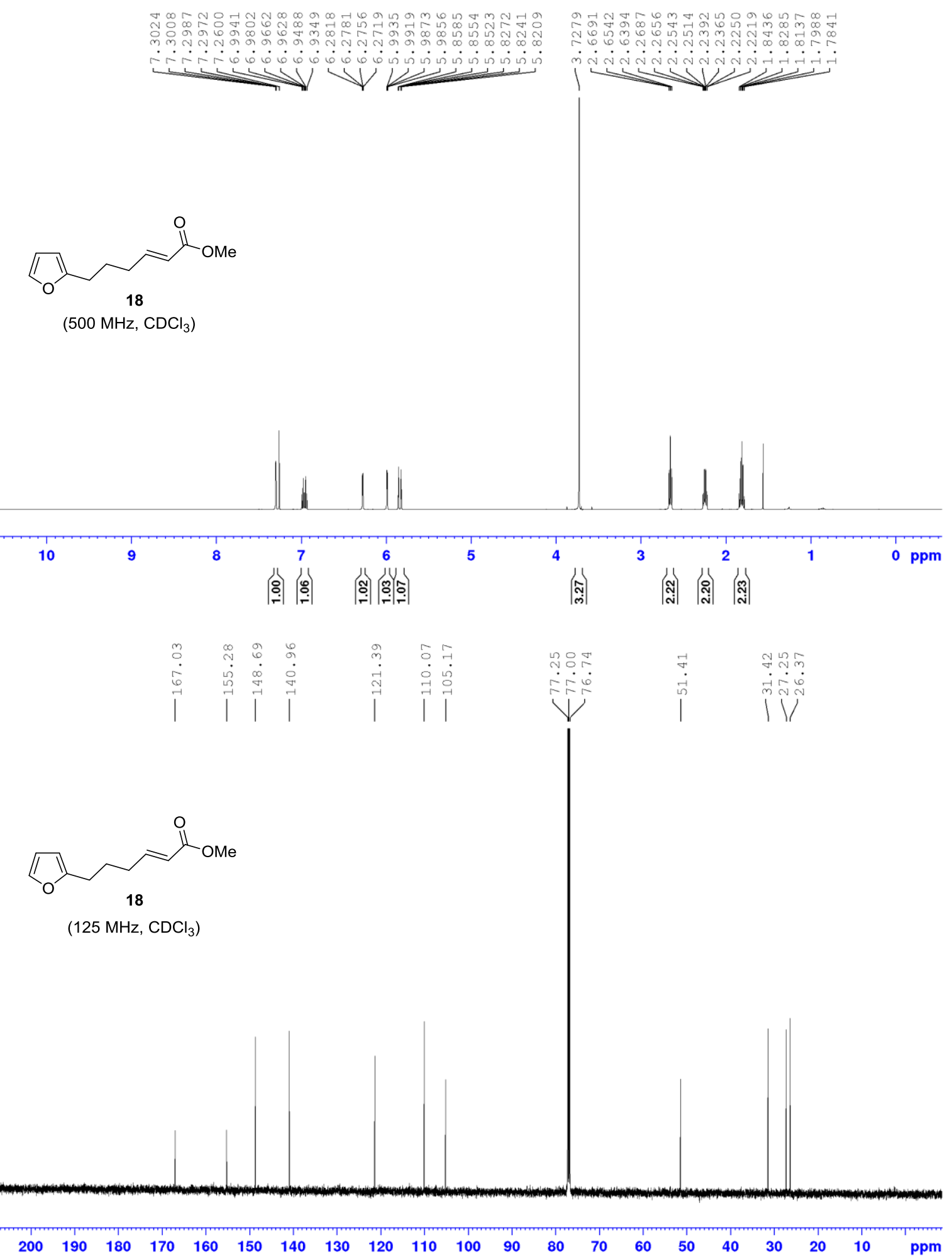

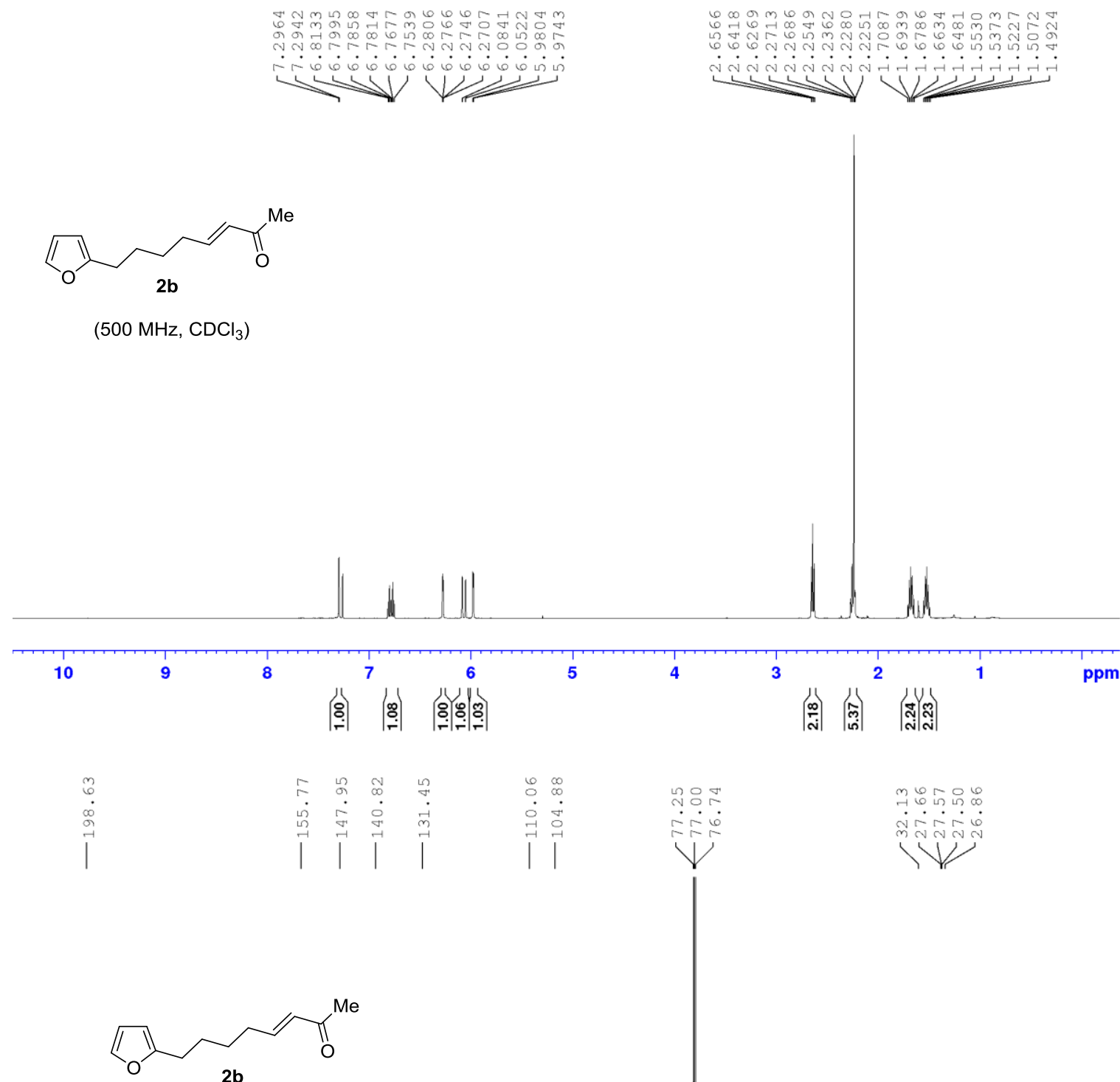

$\left(125 \mathrm{MHz}, \mathrm{CDCl}_{3}\right.$ )

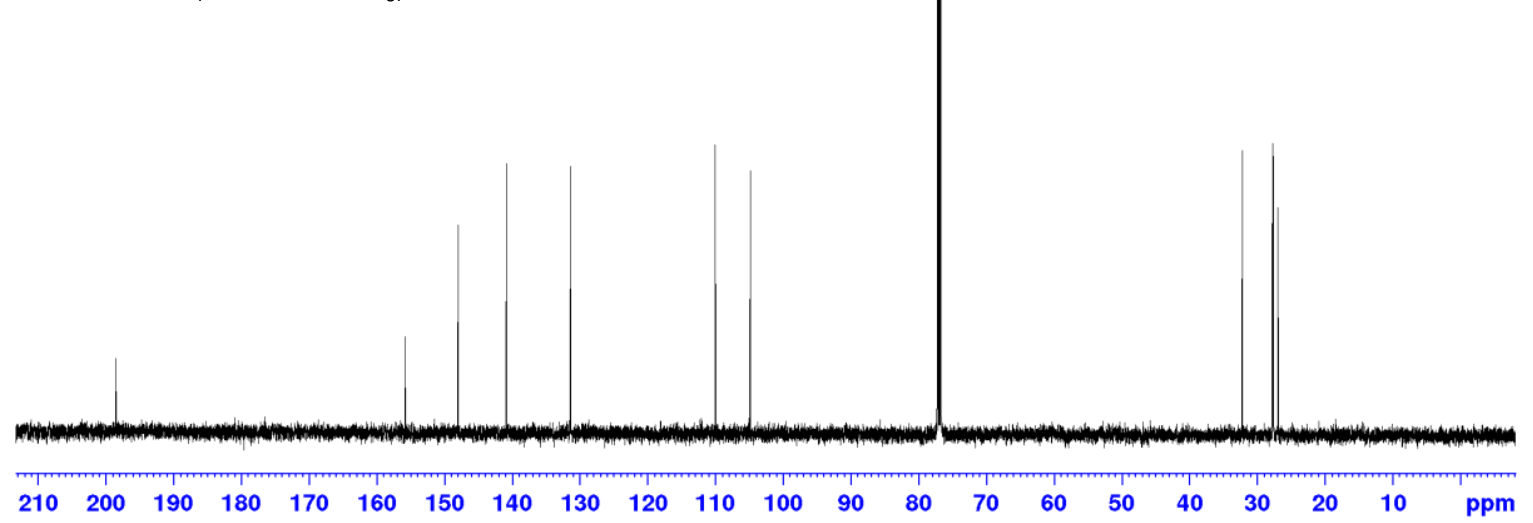




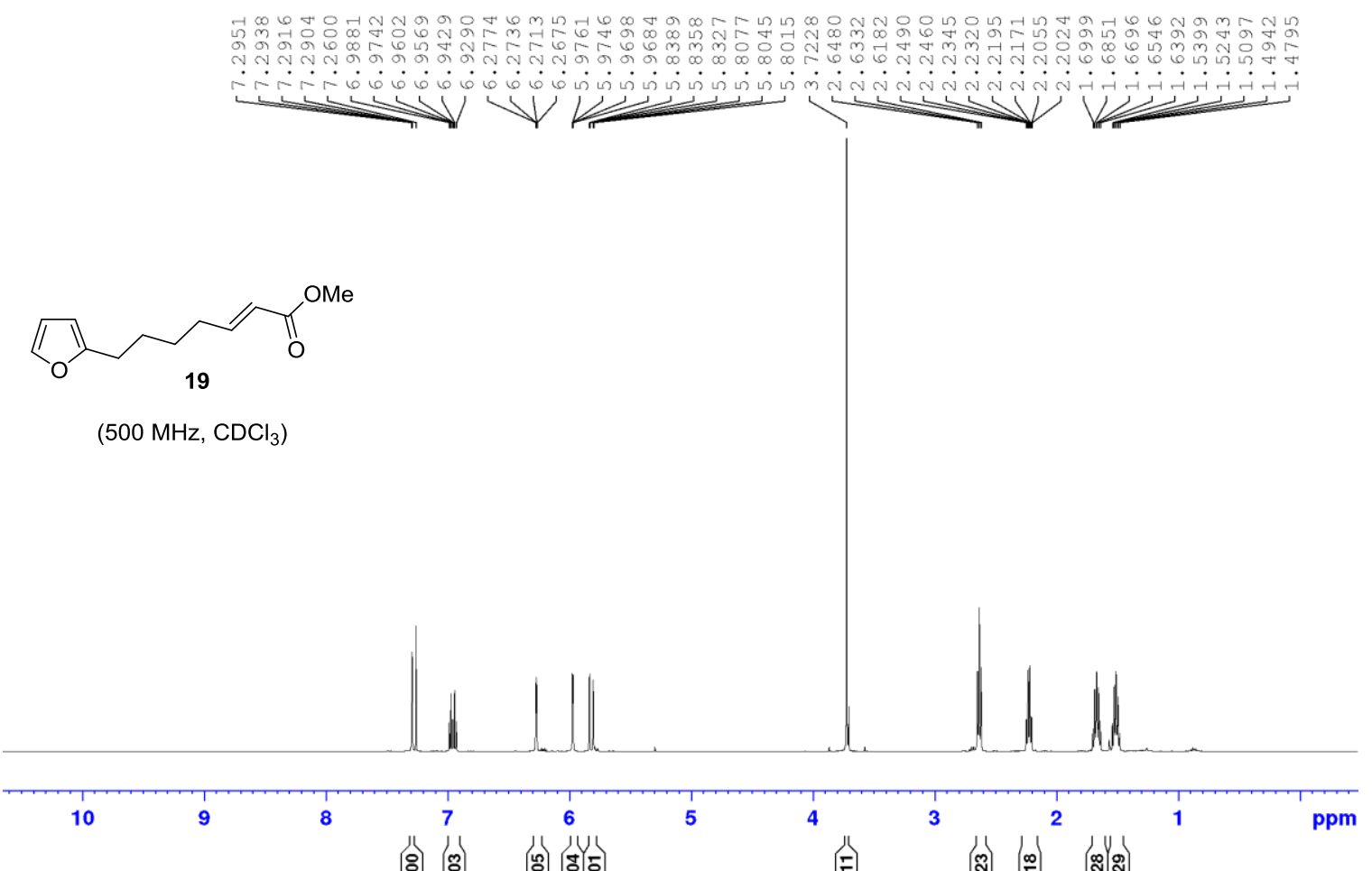
罟|
蓙|
$|\hat{p i p}|$

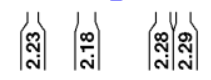

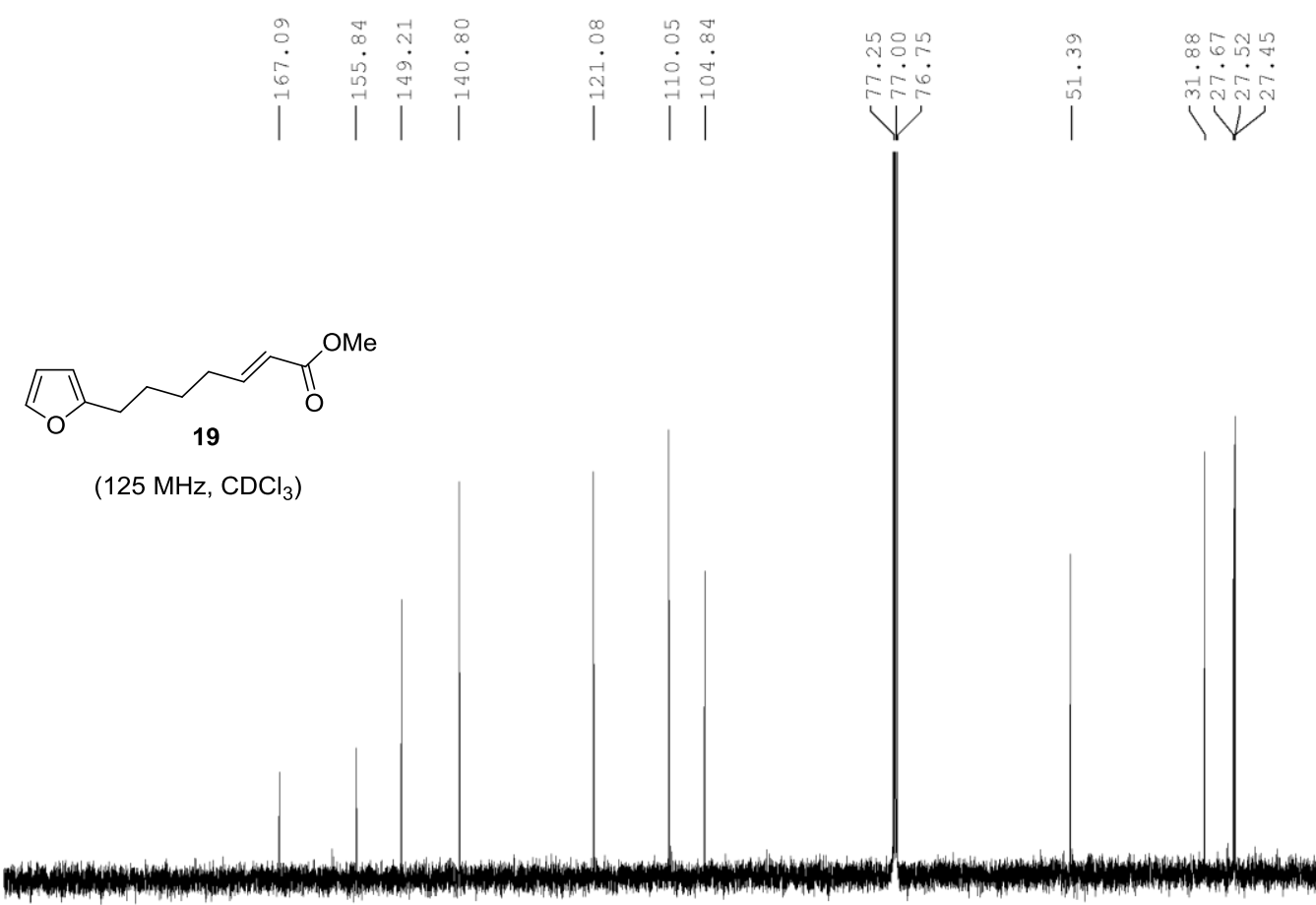

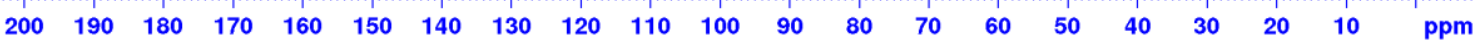




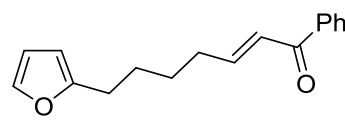

2c

(500 MHz, $\mathrm{CDCl}_{3}$ )

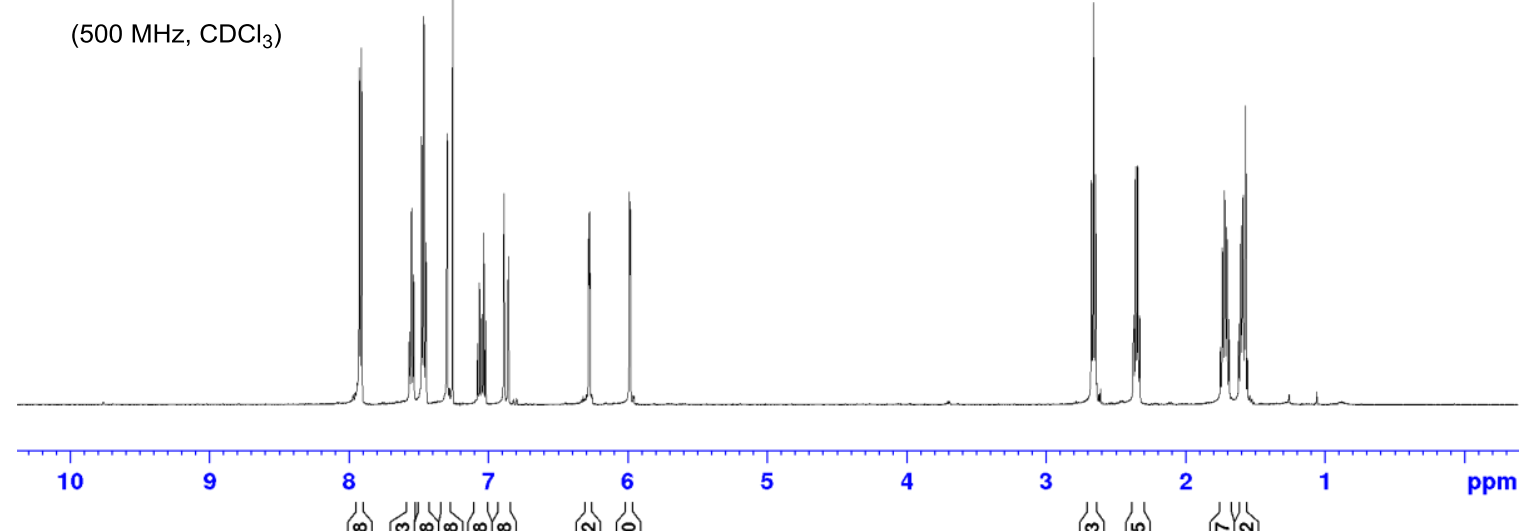

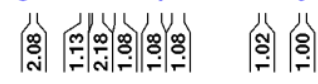

|ึิง

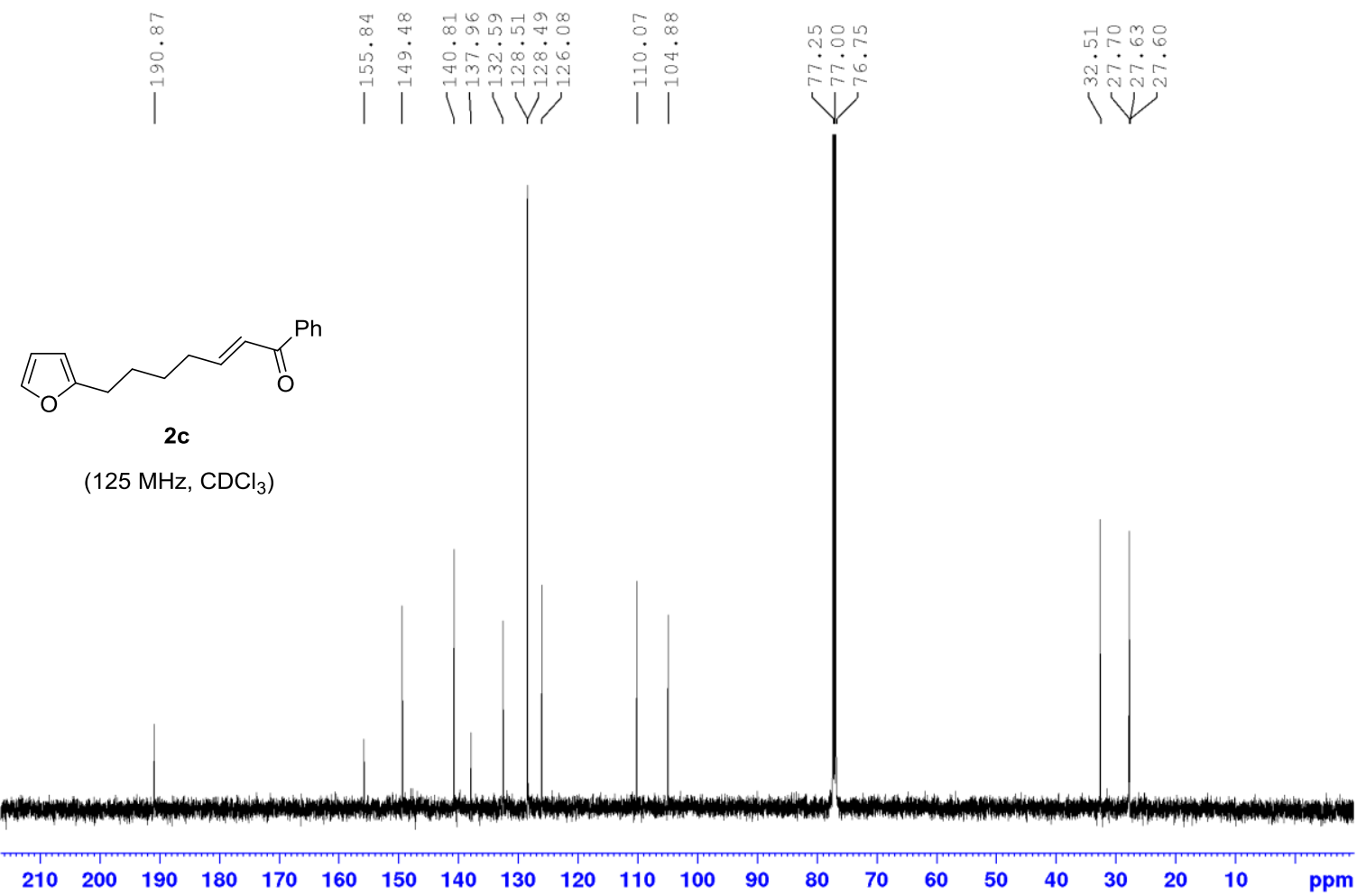




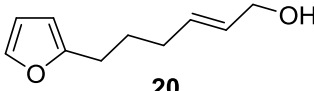

20

$\left(500 \mathrm{MHz}, \mathrm{CDCl}_{3}\right.$ )

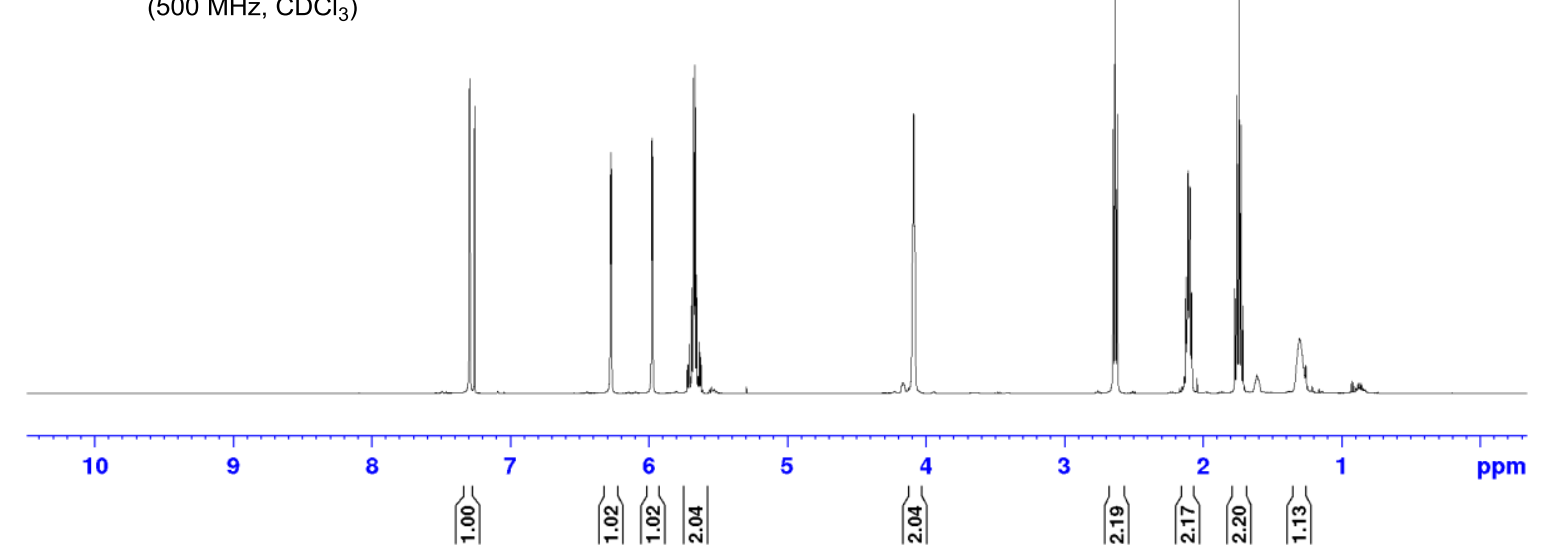

| (a)

|ิ

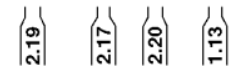

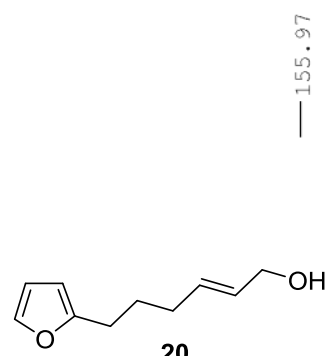

|

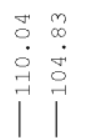

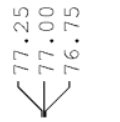

में ले

(125 MHz, $\mathrm{CDCl}_{3}$ )

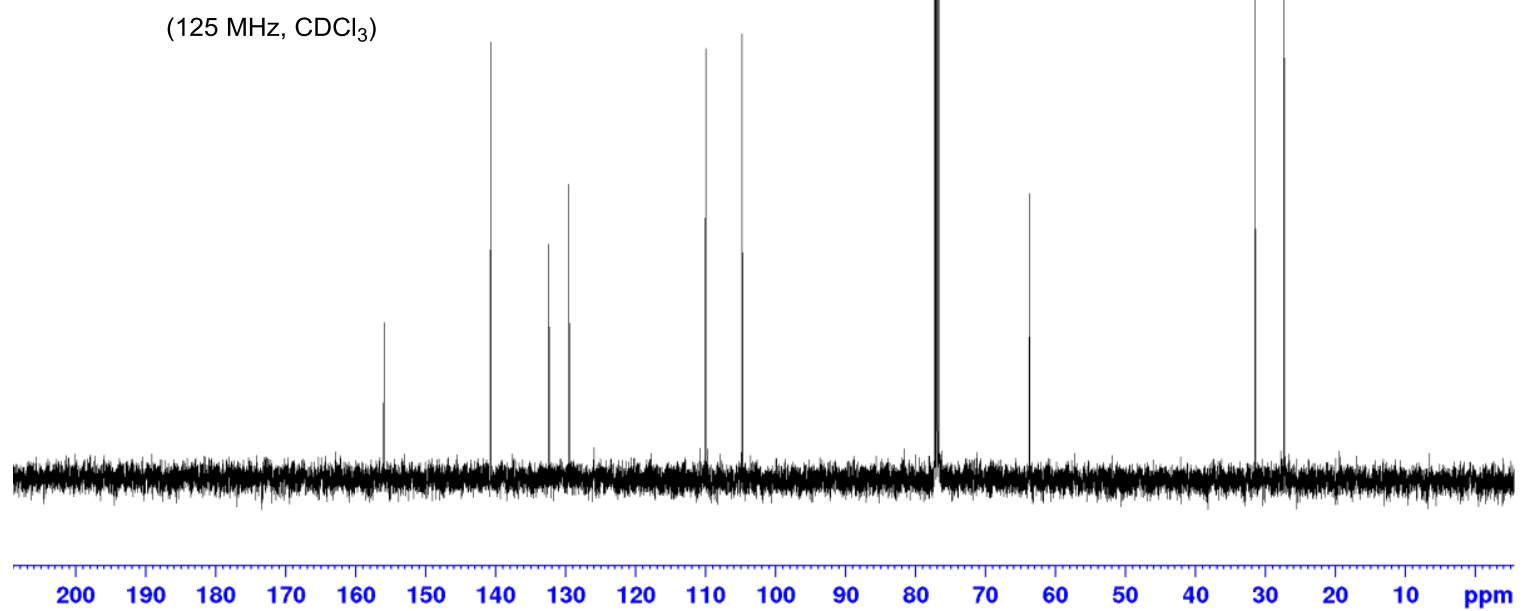




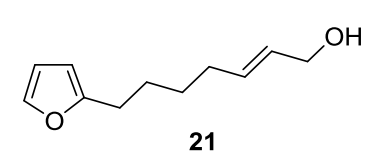

$\left(500 \mathrm{MHz}, \mathrm{CDCl}_{3}\right.$ )
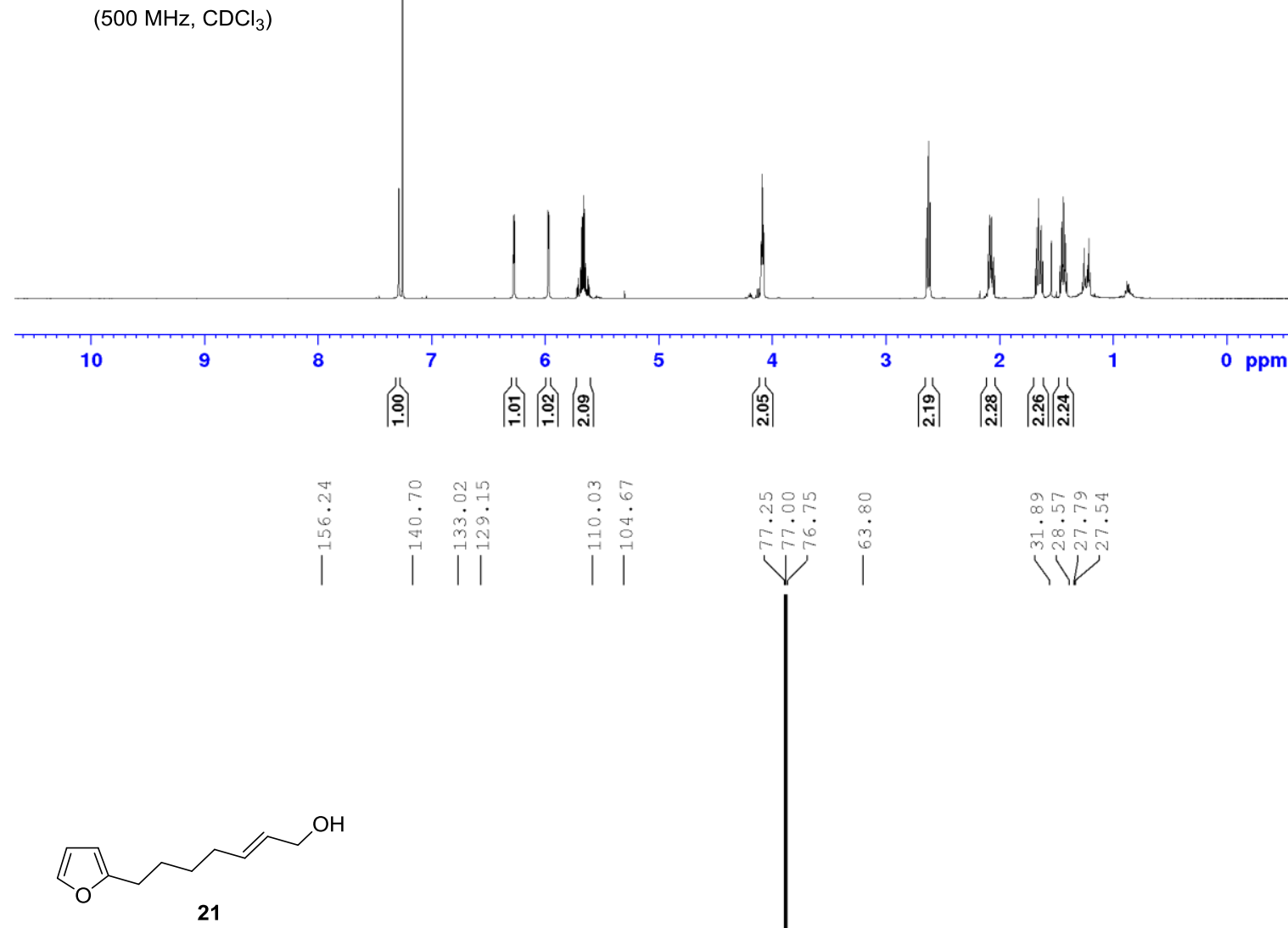

(125 MHz, $\mathrm{CDCl}_{3}$ )
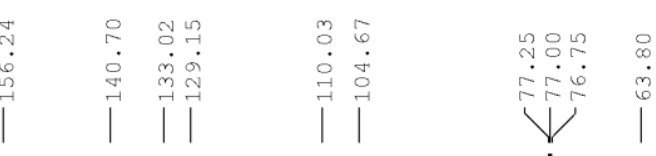

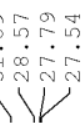

(125

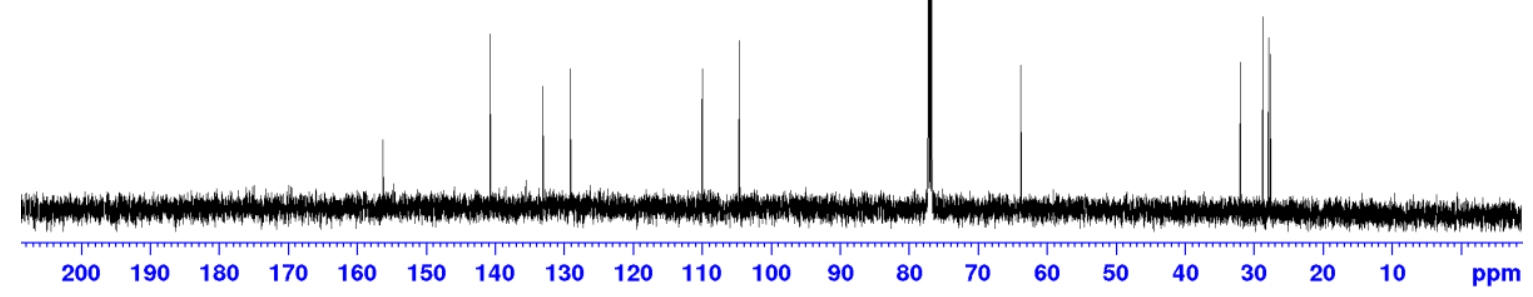



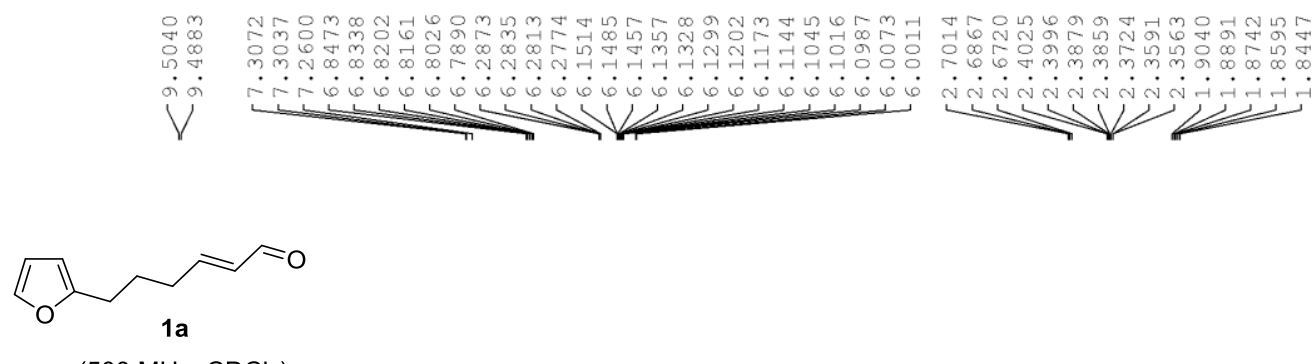

$\left(500 \mathrm{MHz}, \mathrm{CDCl}_{3}\right)$
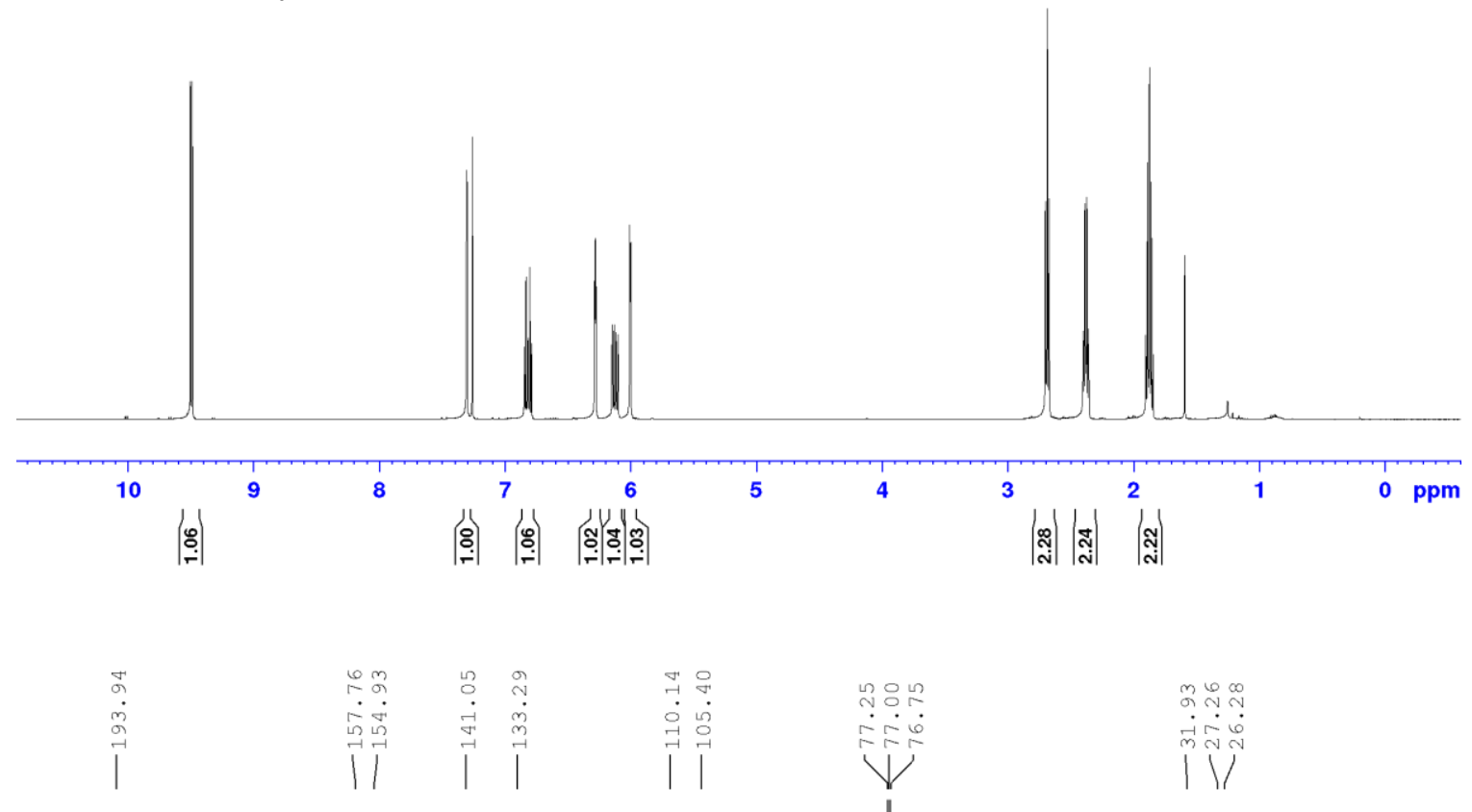

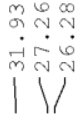

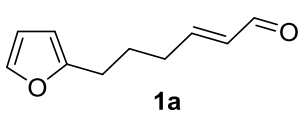

$\left(125 \mathrm{MHz}^{\mathrm{CDCl}}\right)_{3}$

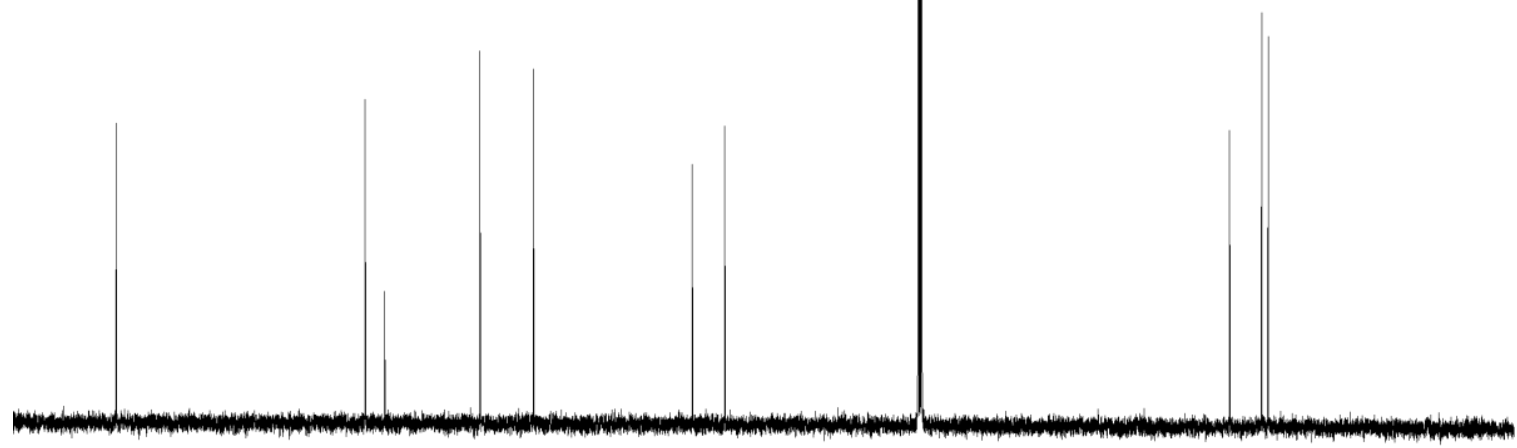

$\begin{array}{llllllllllllllllllllll}200 & 190 & 180 & 170 & 160 & 150 & 140 & 130 & 120 & 110 & 100 & 90 & 80 & 70 & 60 & 50 & 40 & 30 & 20 & 10 & \mathrm{ppm}\end{array}$ 


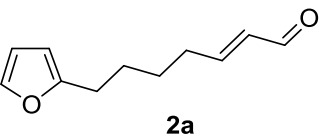

(500 MHz, $\mathrm{CDCl}_{3}$ )
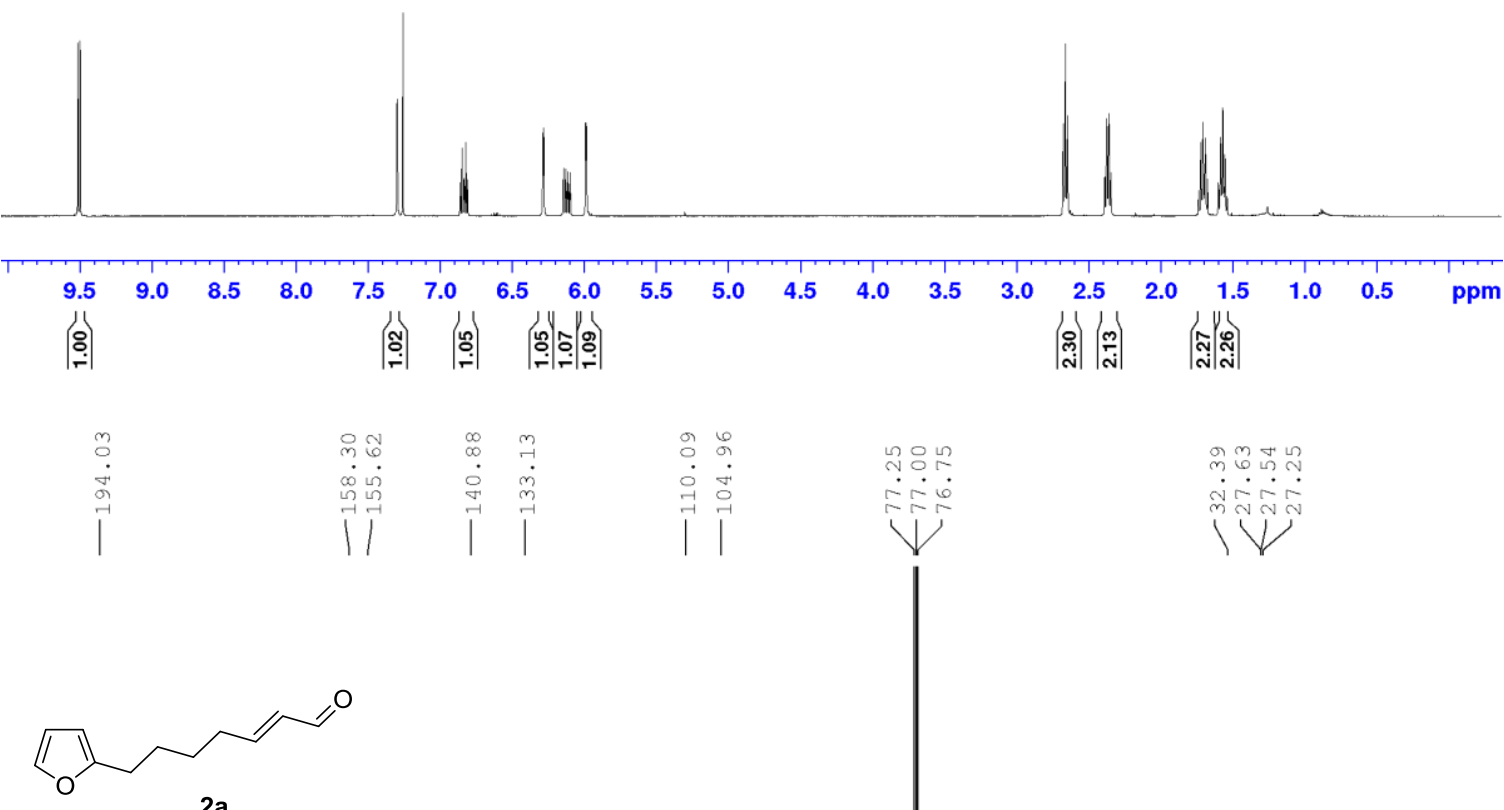

$\left(125 \mathrm{MHz}, \mathrm{CDCl}_{3}\right.$ )

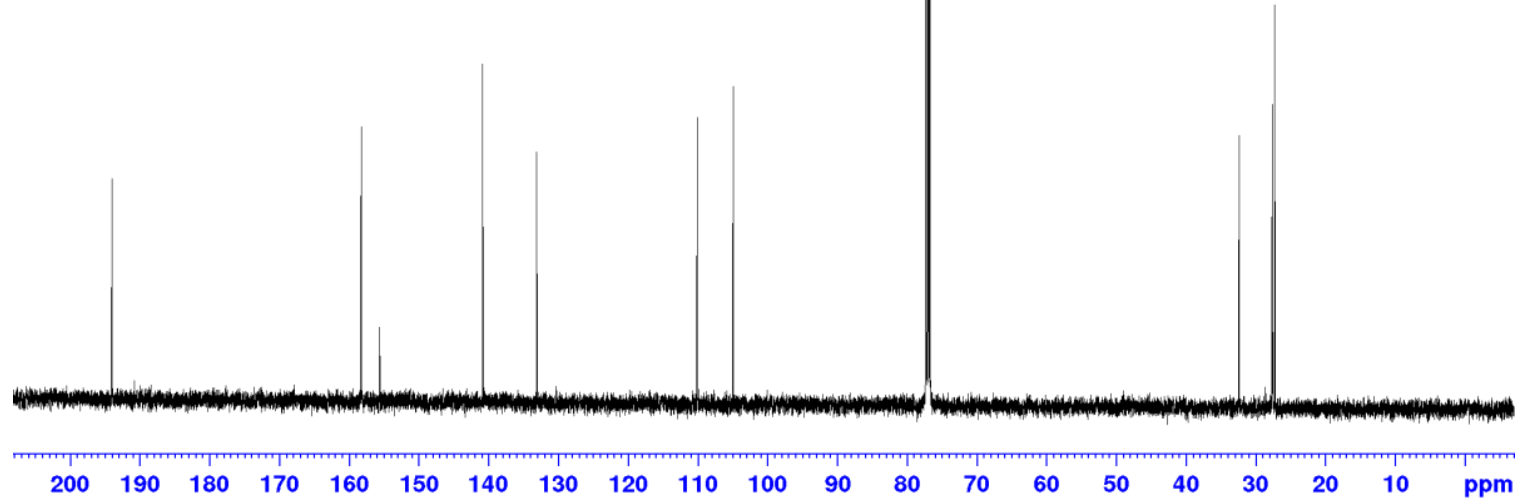




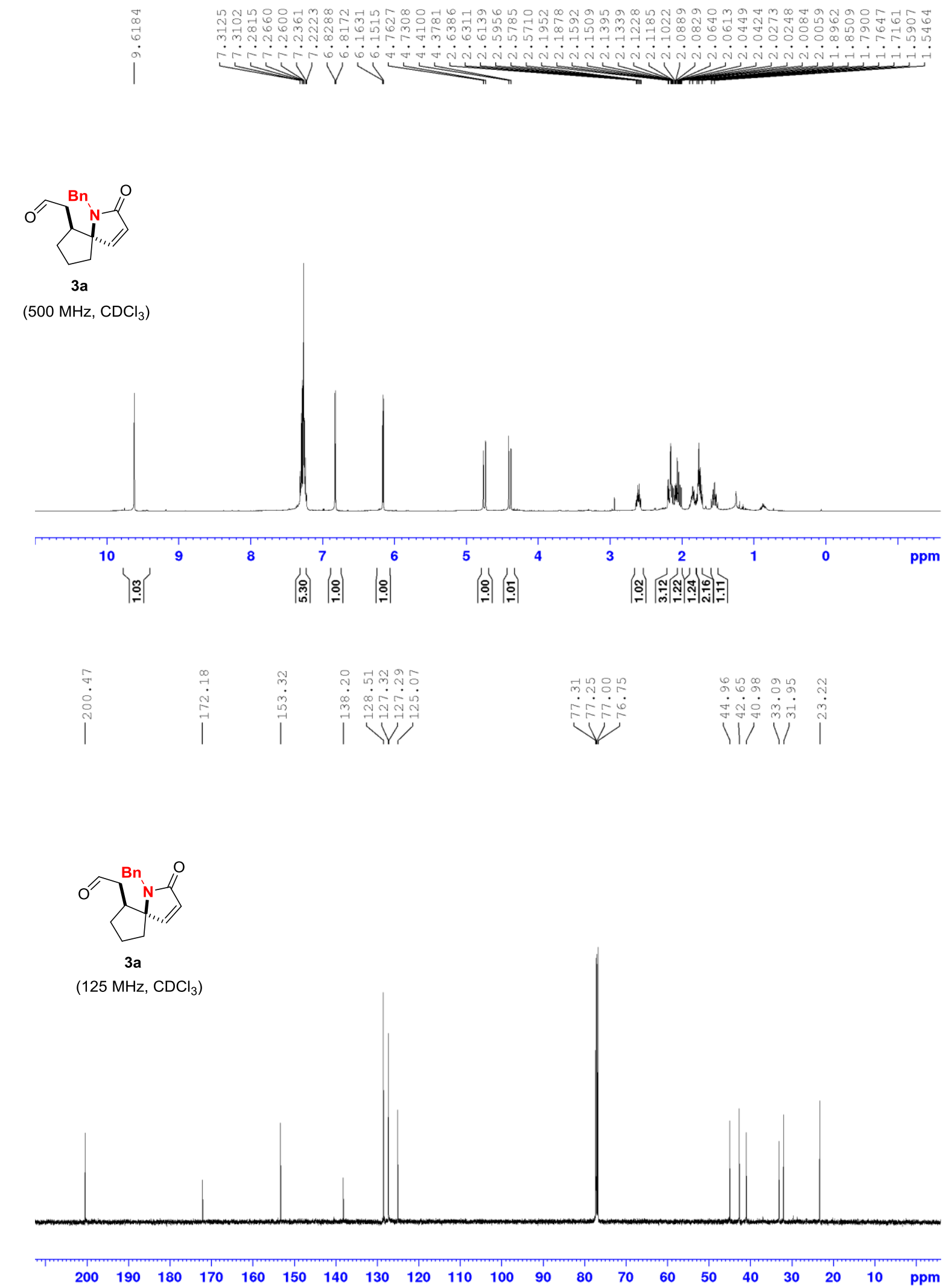




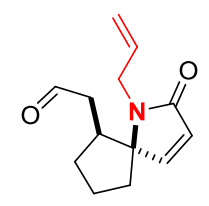

3b

$\left(500 \mathrm{MHz}, \mathrm{CDCl}_{3}\right)$
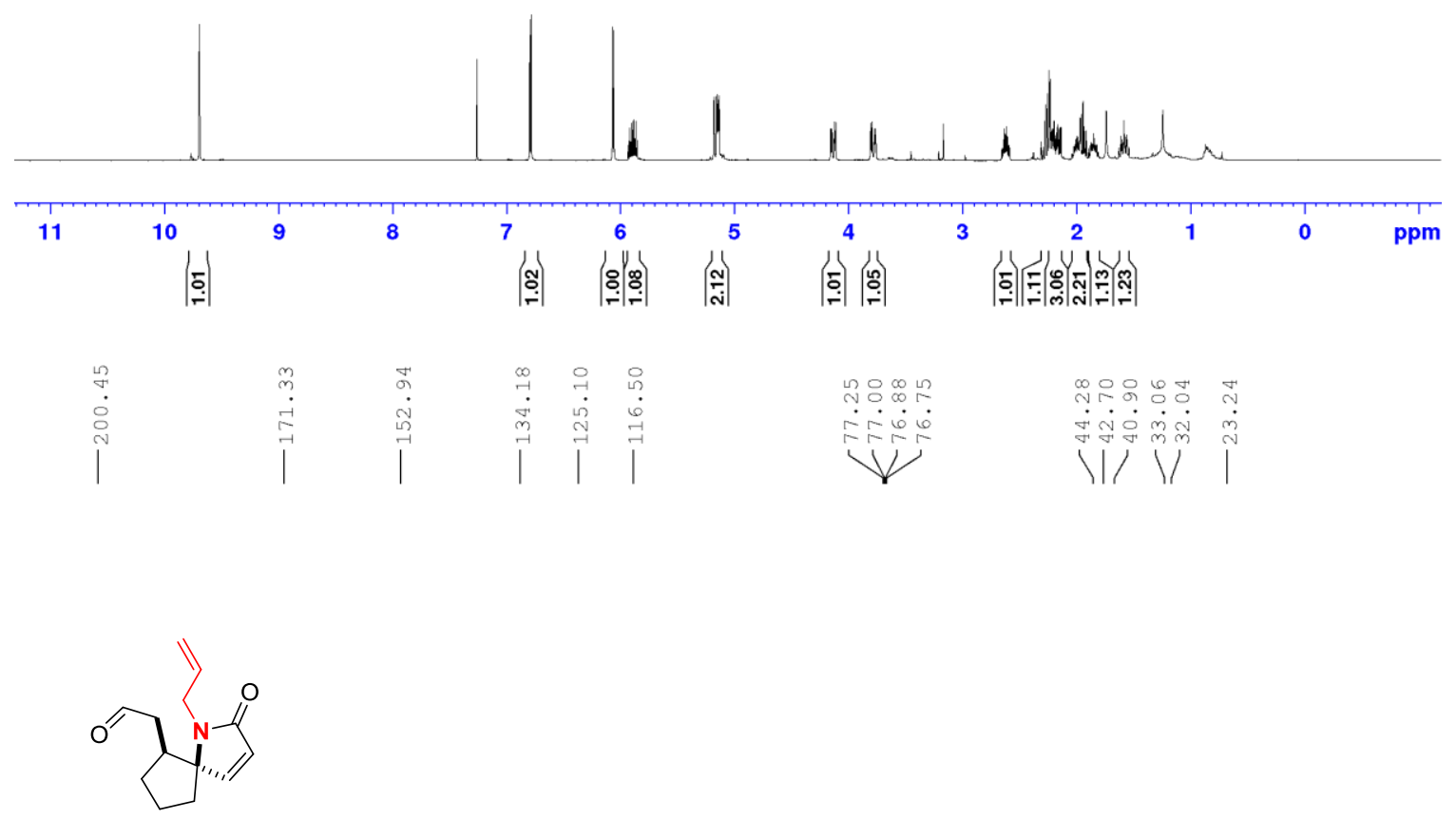

3b

$\left(125 \mathrm{MHz}, \mathrm{CDCl}_{3}\right)$

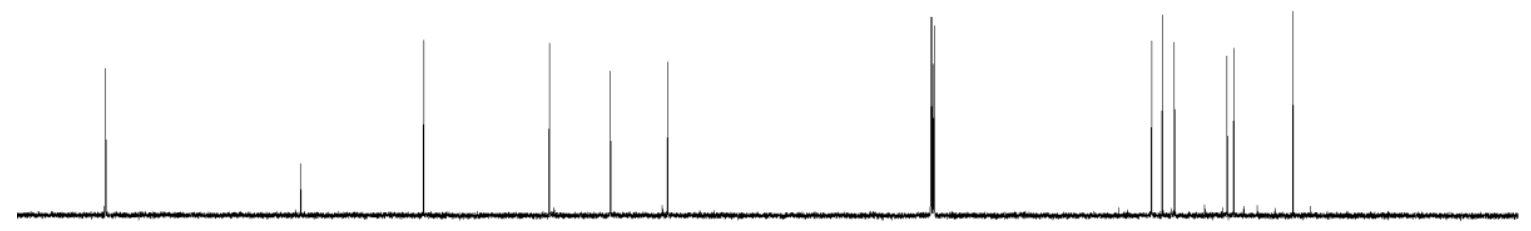

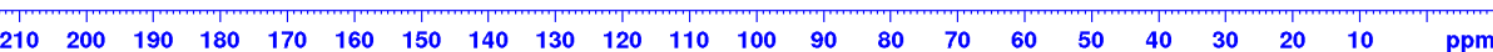



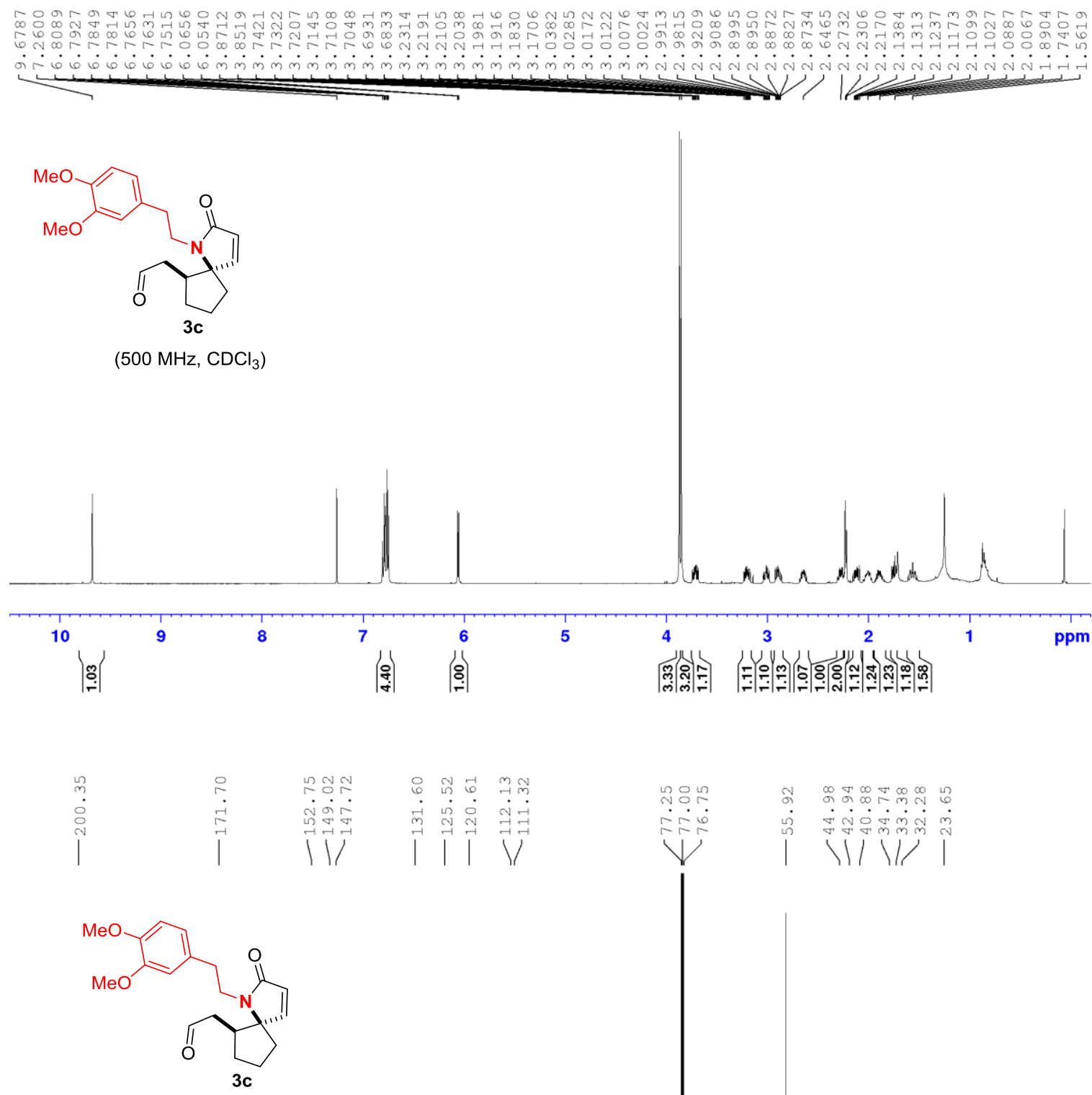

(125 MHz, $\mathrm{CDCl}_{3}$ )

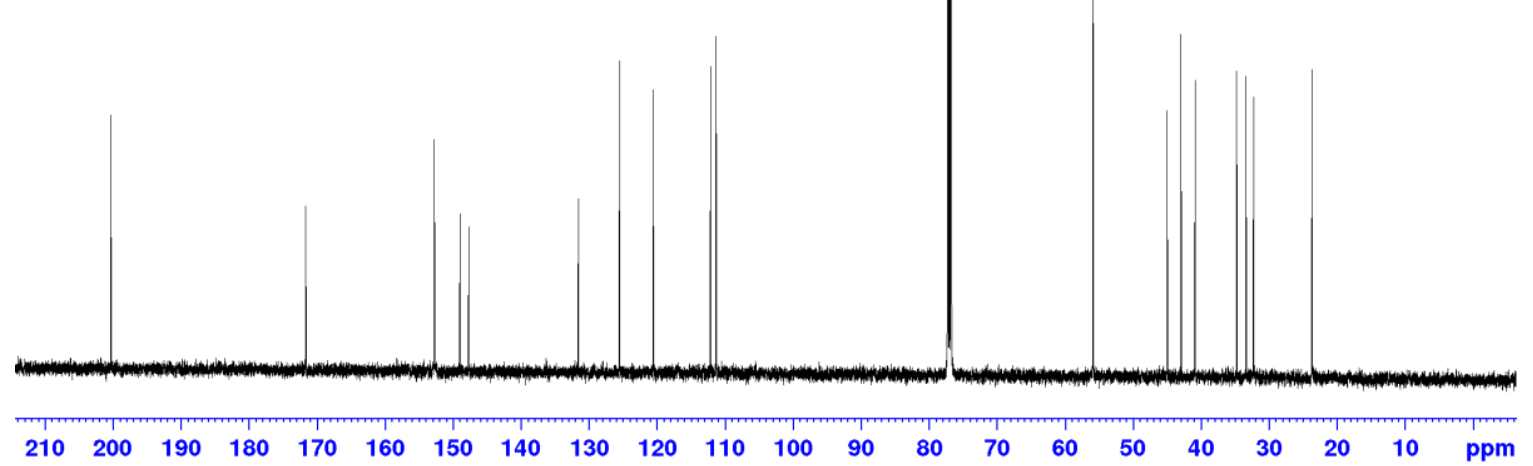




\section{HMBC correlations of compound $3 \mathrm{c}$}

The chemical shift of the $\alpha$-quaternary carbon next to the $\mathrm{N}$-atom was revealed by HMBC.
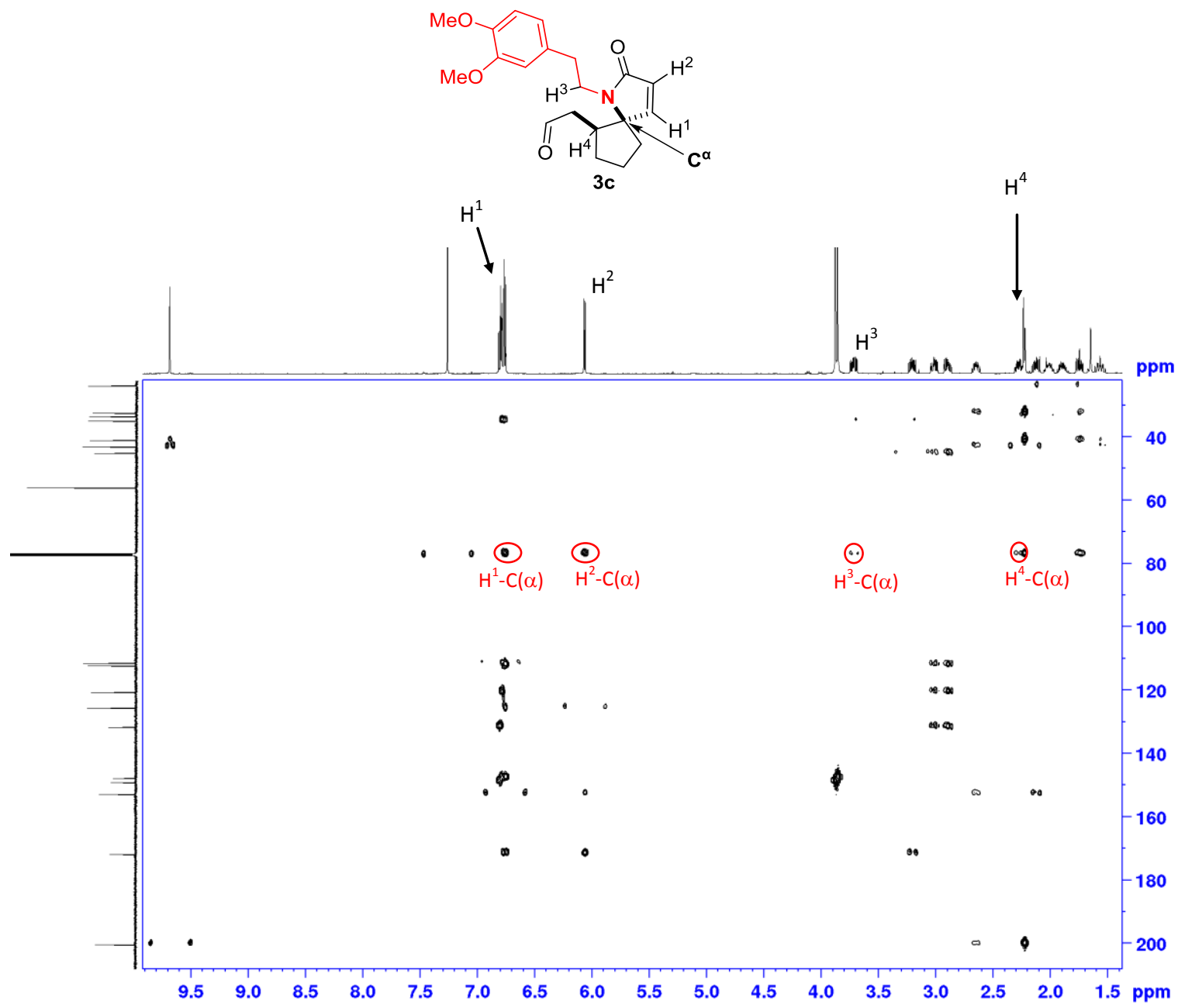

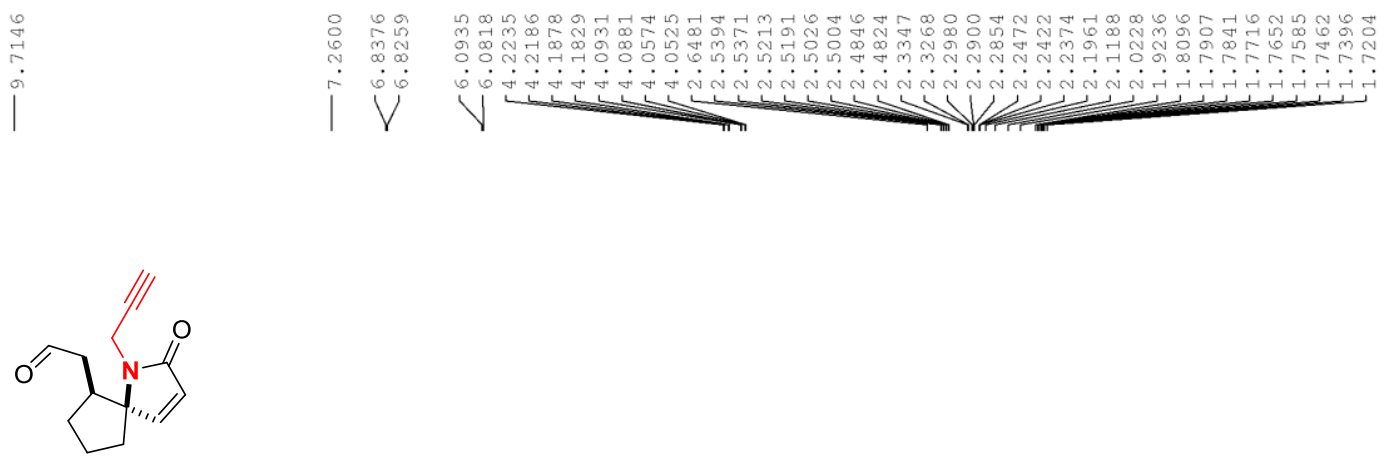

$3 d$

(500 MHz, $\mathrm{CDCl}_{3}$ )
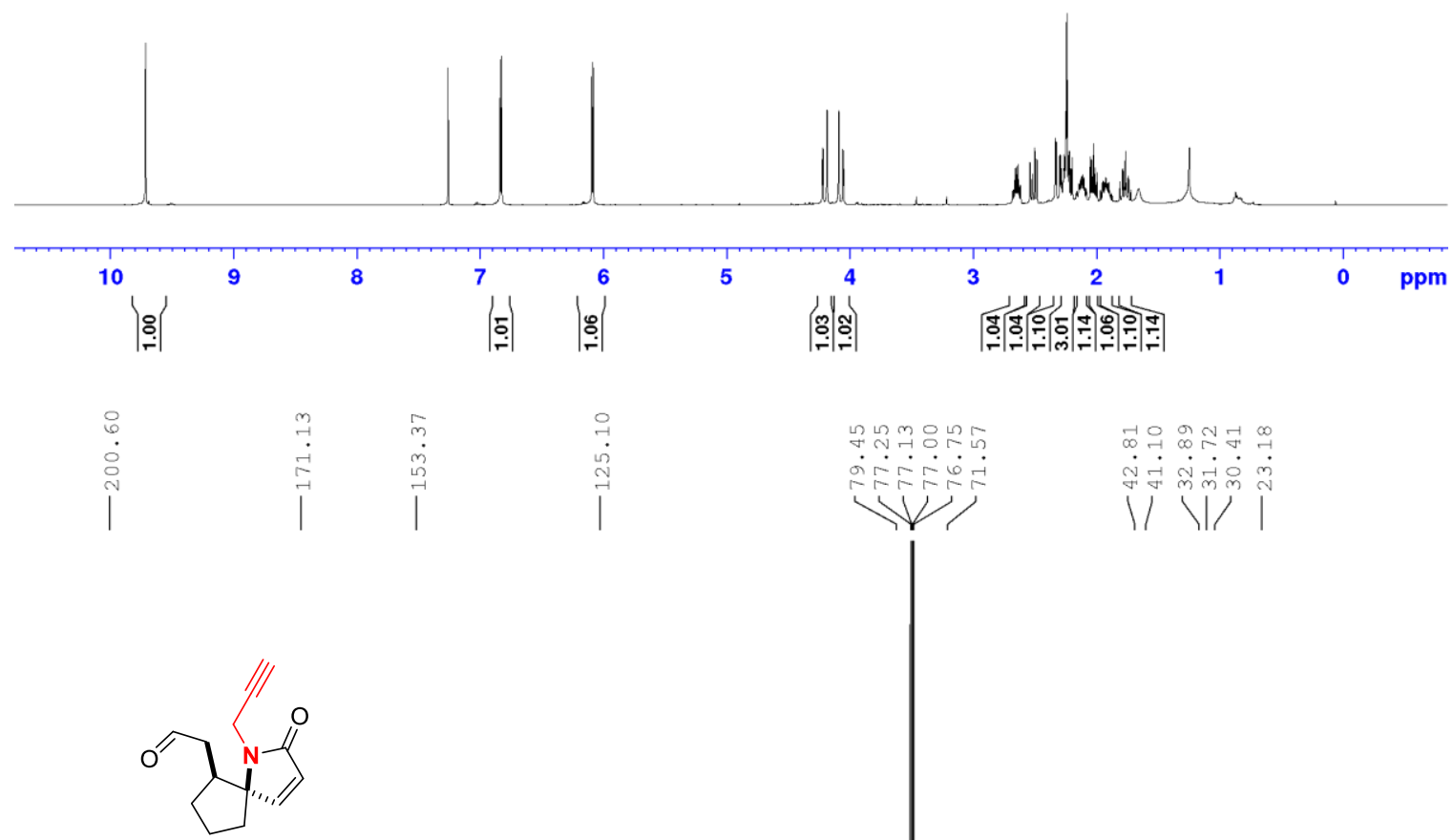

3d

(125 $\mathrm{MHz} \mathrm{CDCl}_{3}$ )

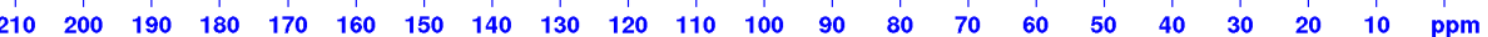


COSY correlations of compound 3d

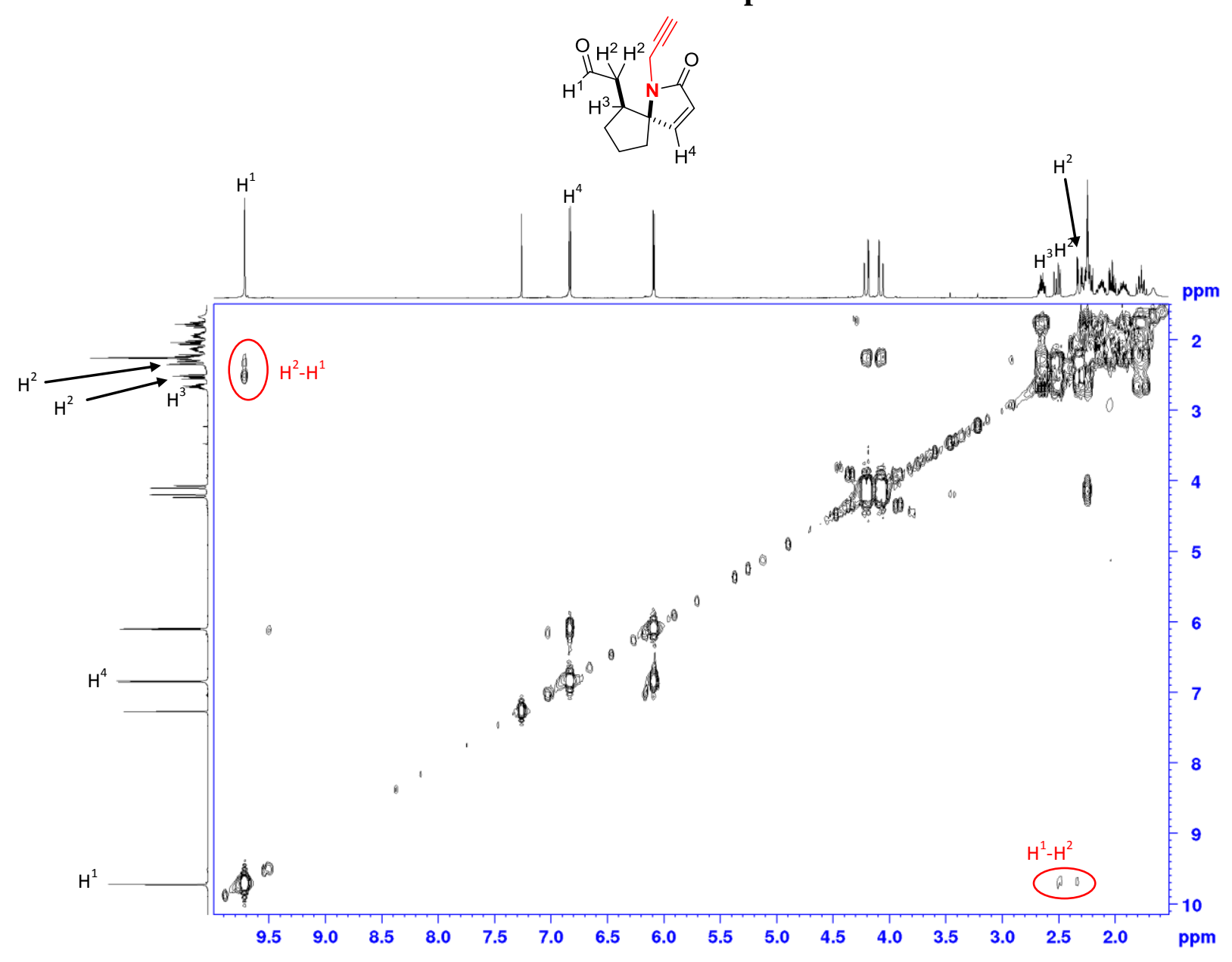


HSQC correlations of compound 3d

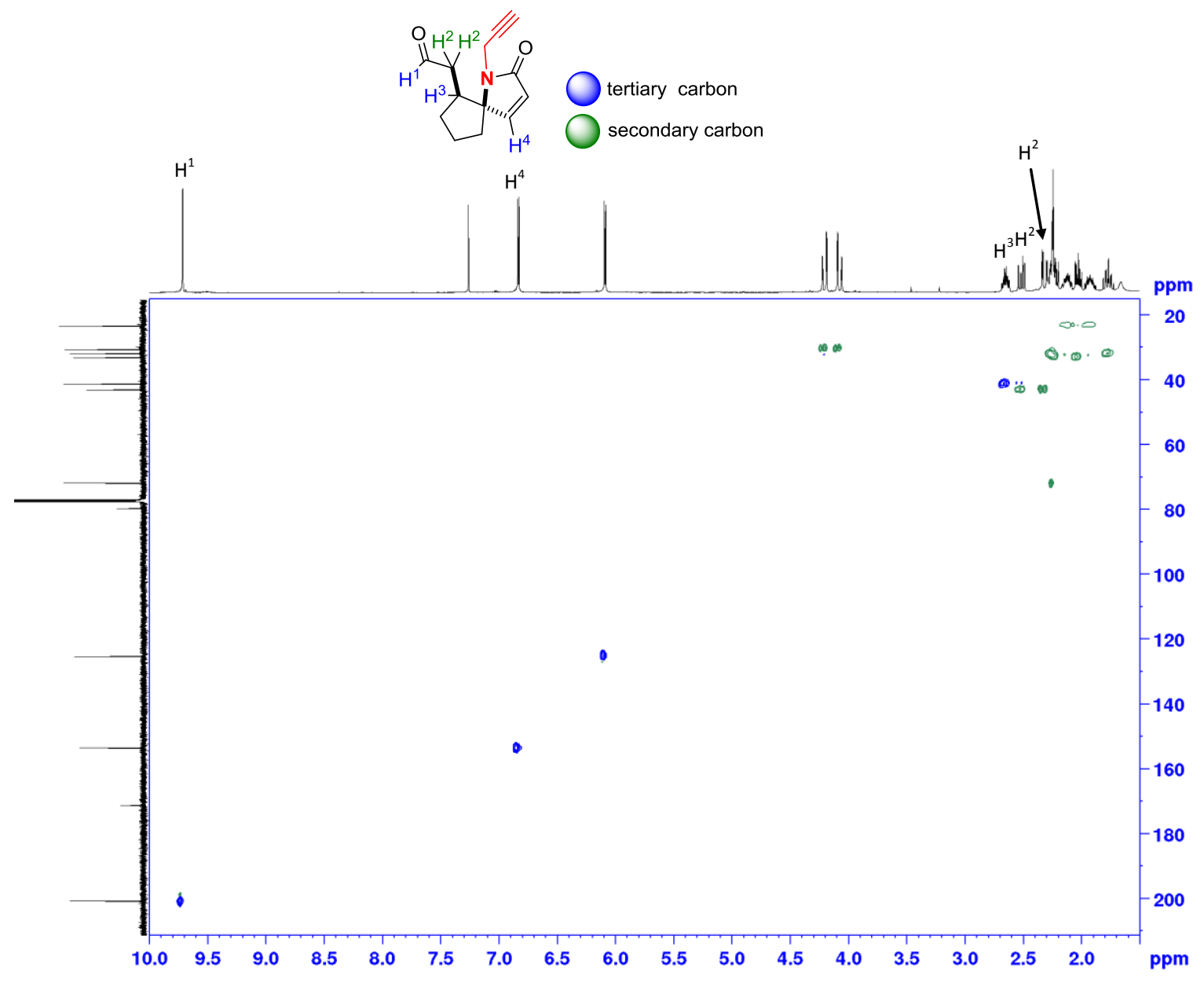


HMBC correlations of compound $3 d$

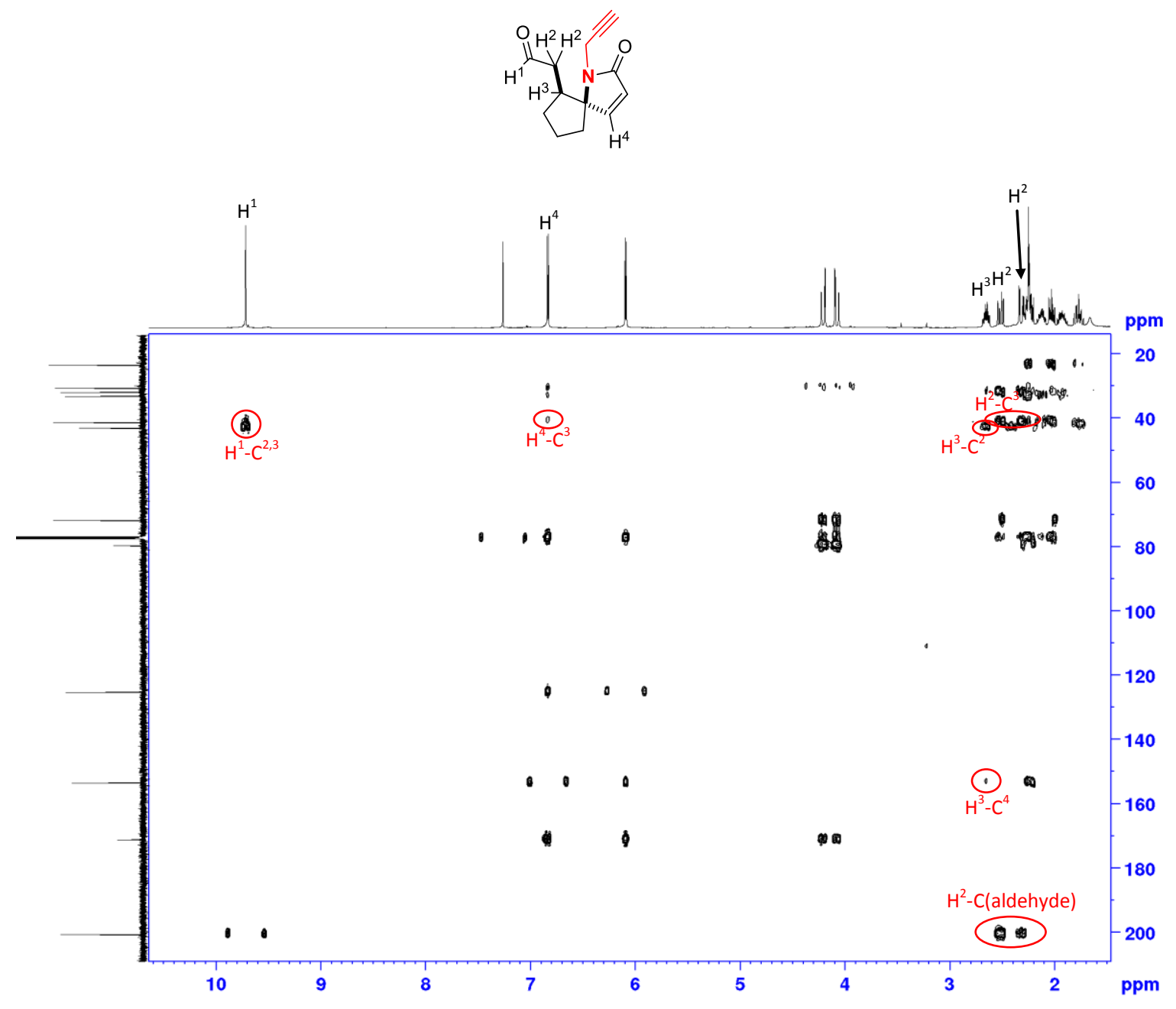


Representative NOEs of compound 3d
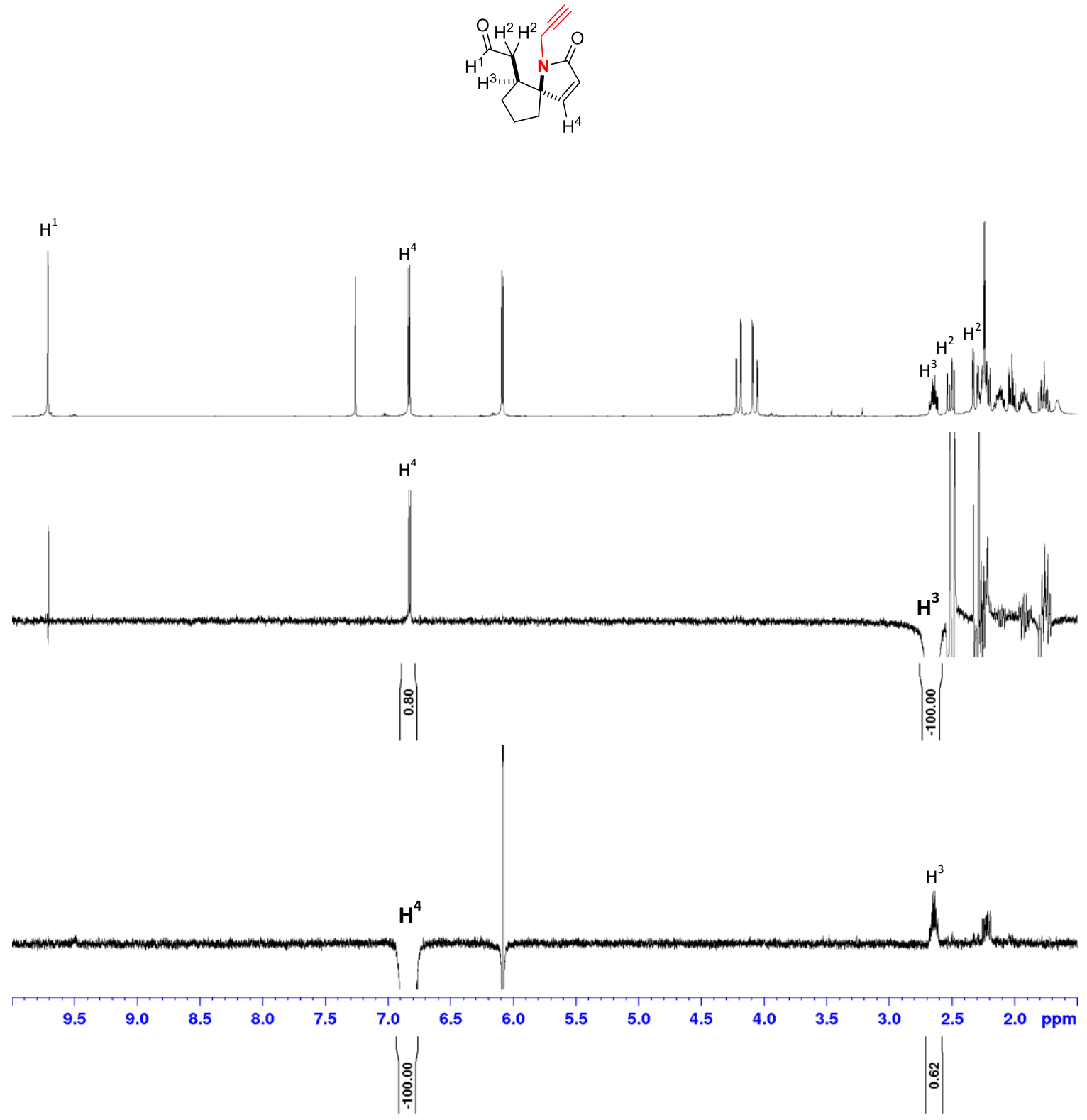


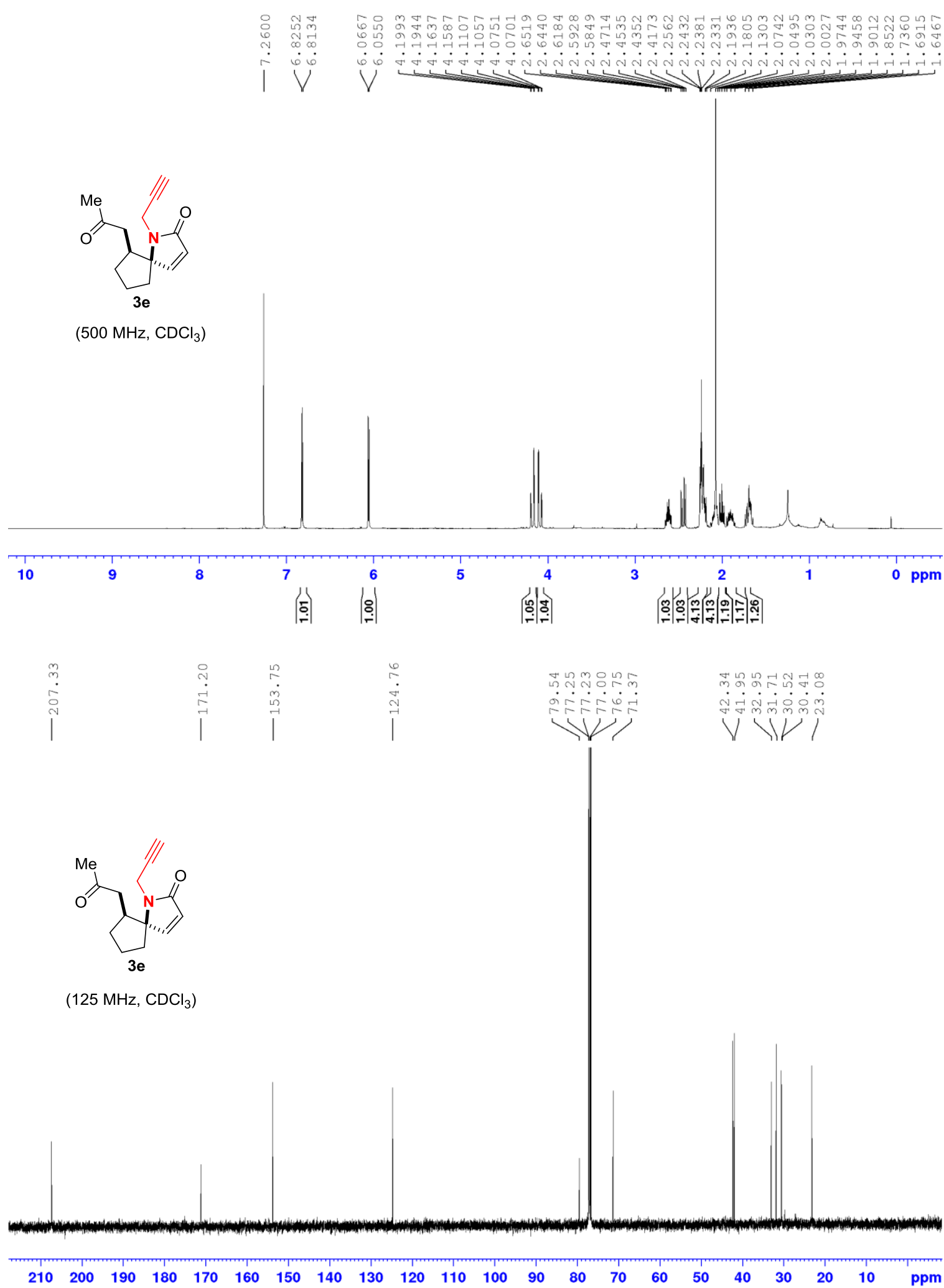



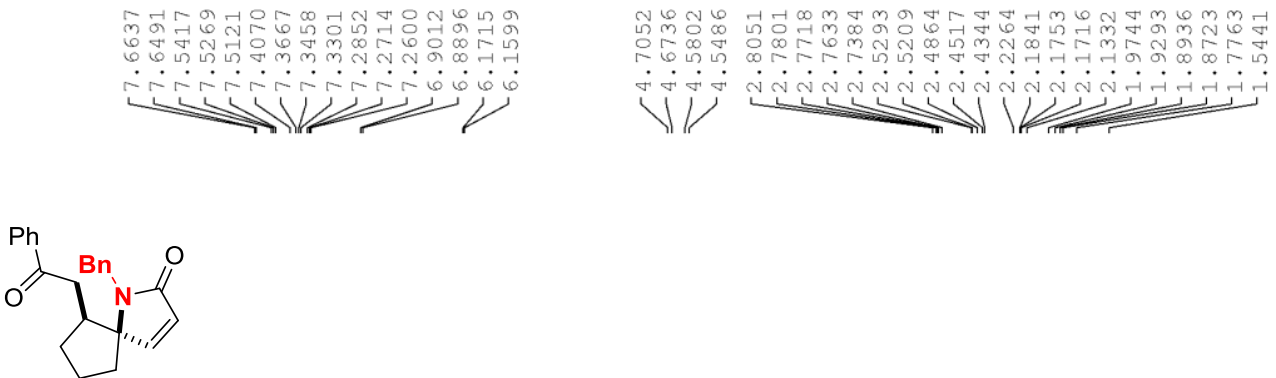

$3 f$

$\left(500 \mathrm{MHz}, \mathrm{CDCl}_{3}\right)$

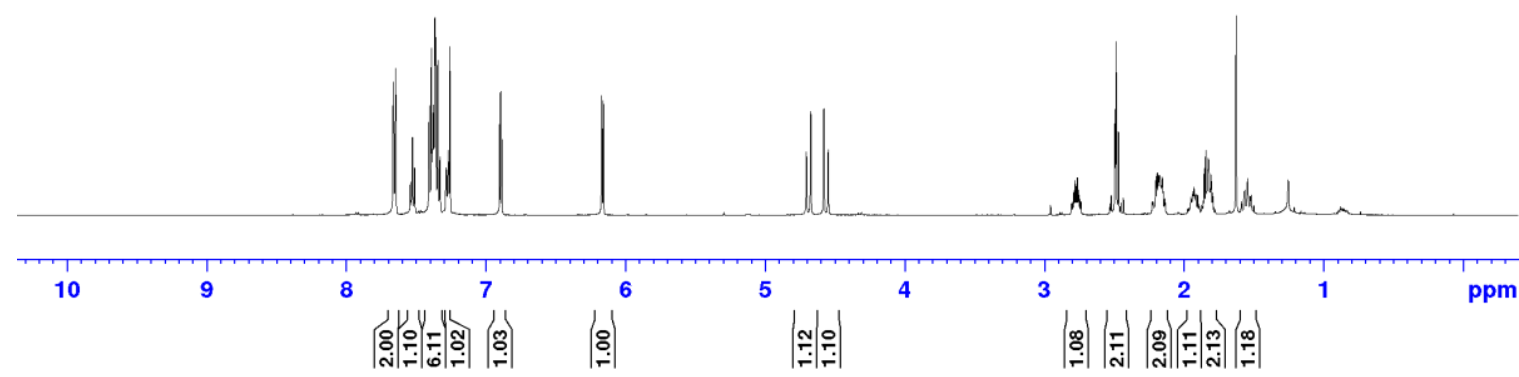

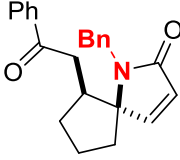

$3 f$

(125 MHz, $\mathrm{CDCl}_{3}$ )

|

pm 


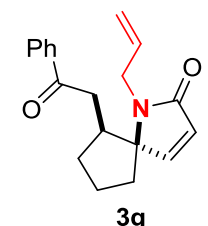

$\left(500 \mathrm{MHz}, \mathrm{CDCl}_{3}\right.$ )

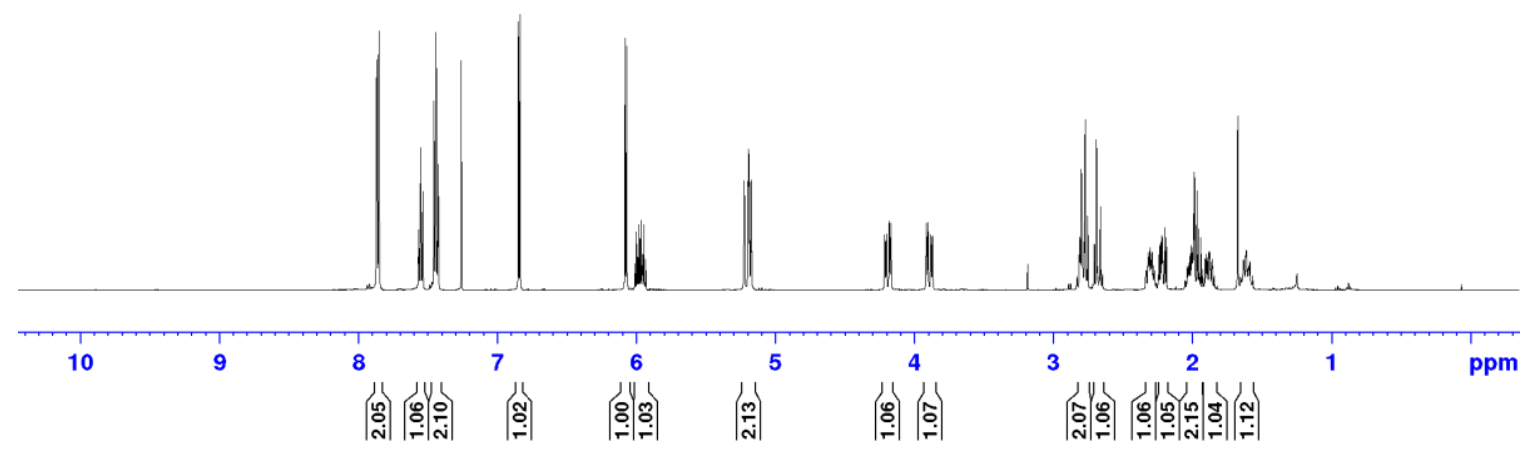

|

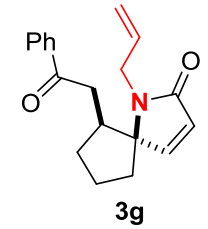

(125 MHz, $\mathrm{CDCl}_{3}$ )

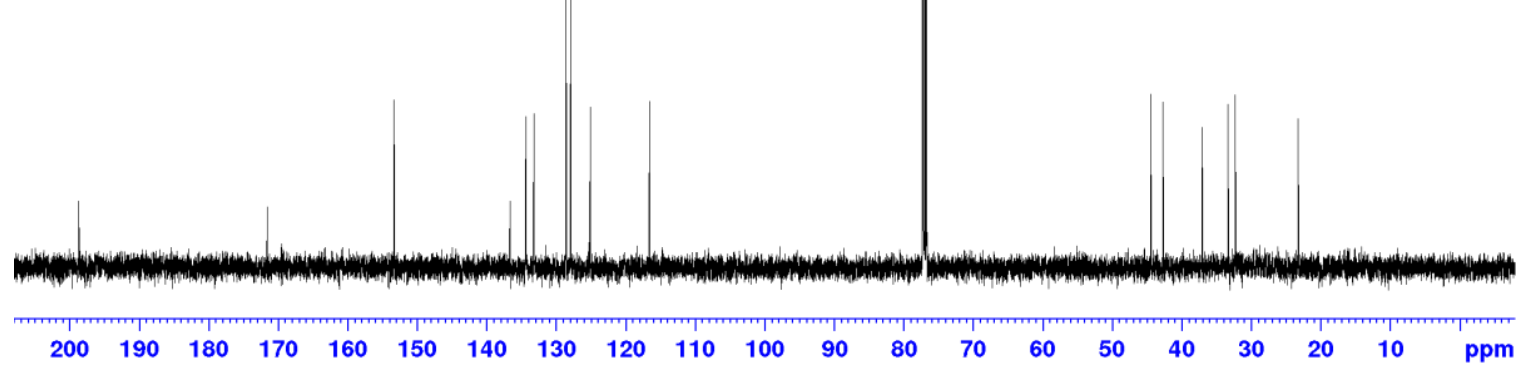




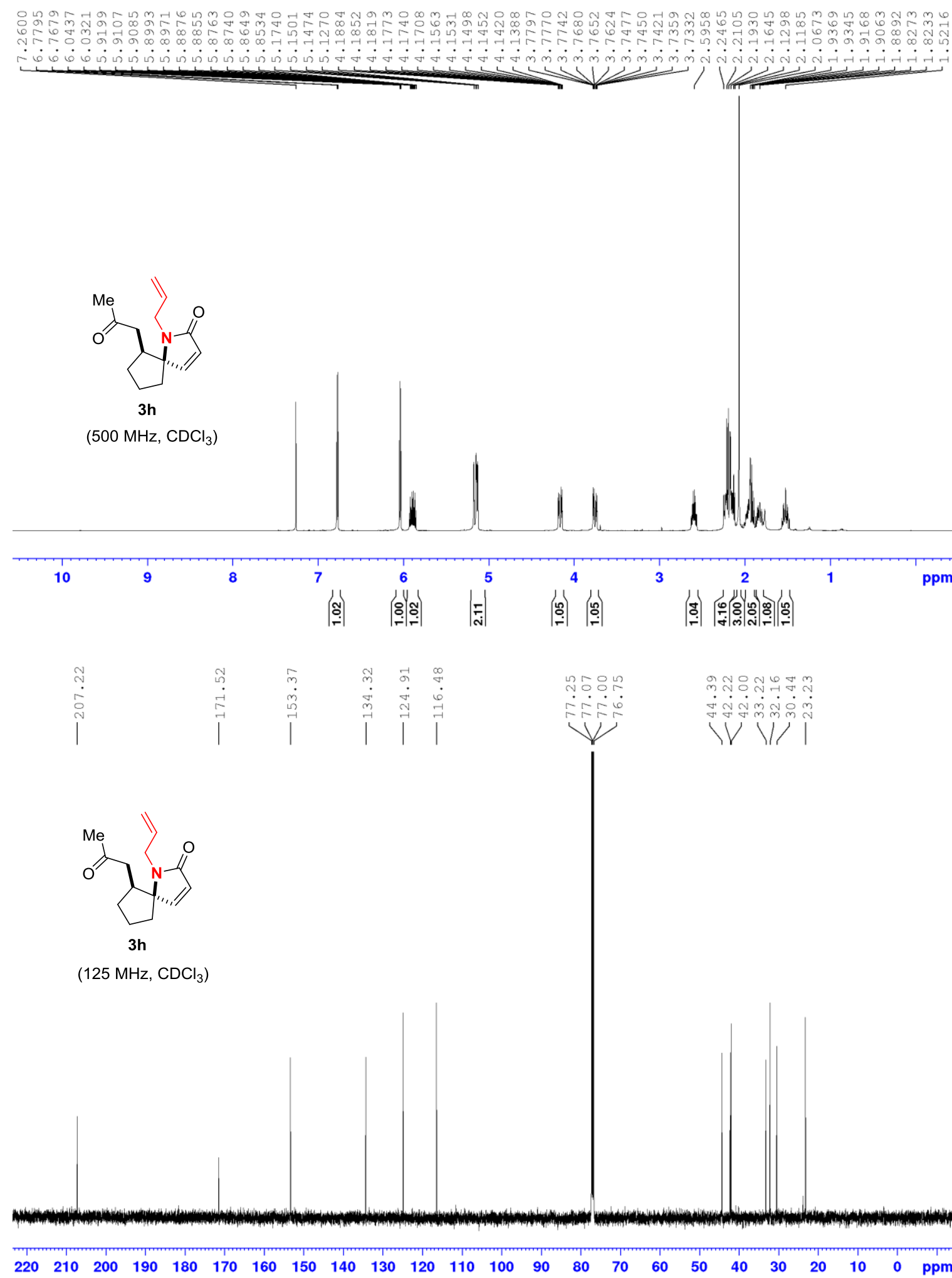



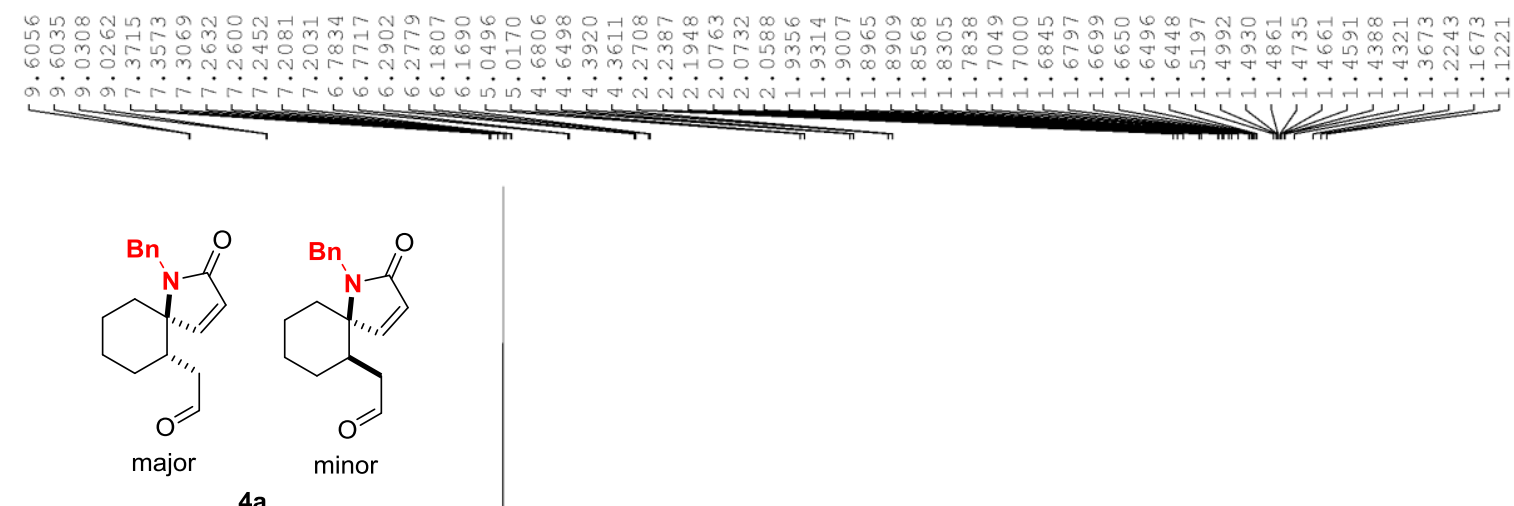

(500 MHz, $\mathrm{CDCl}_{3}$ )
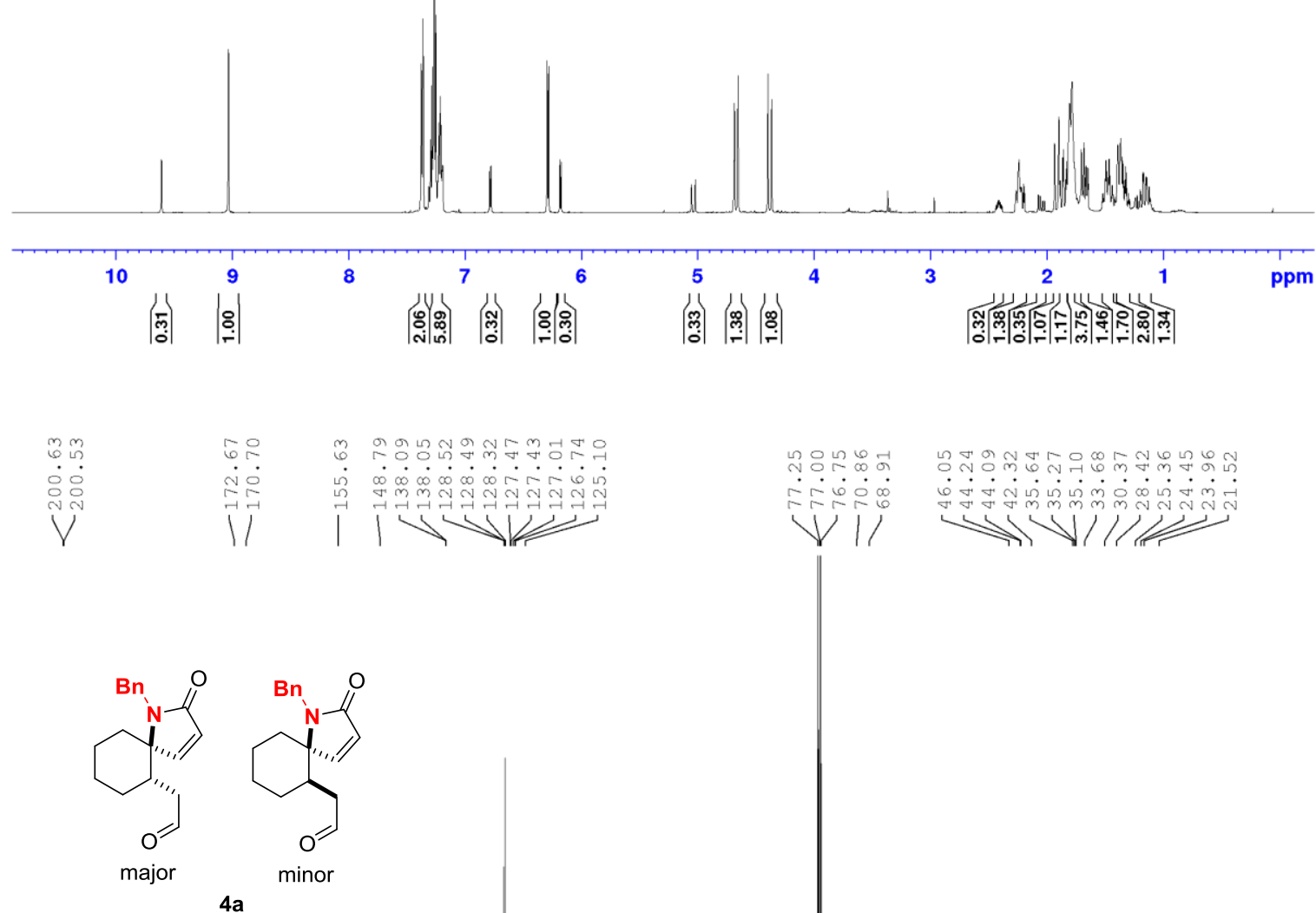

(125 MHz, $\mathrm{CDCl}_{3}$ )

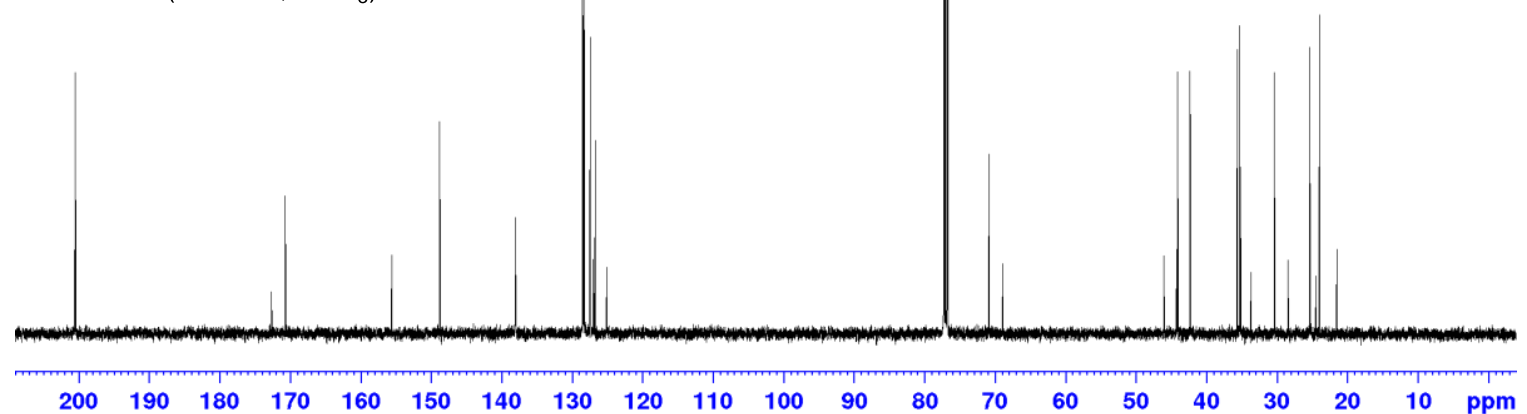




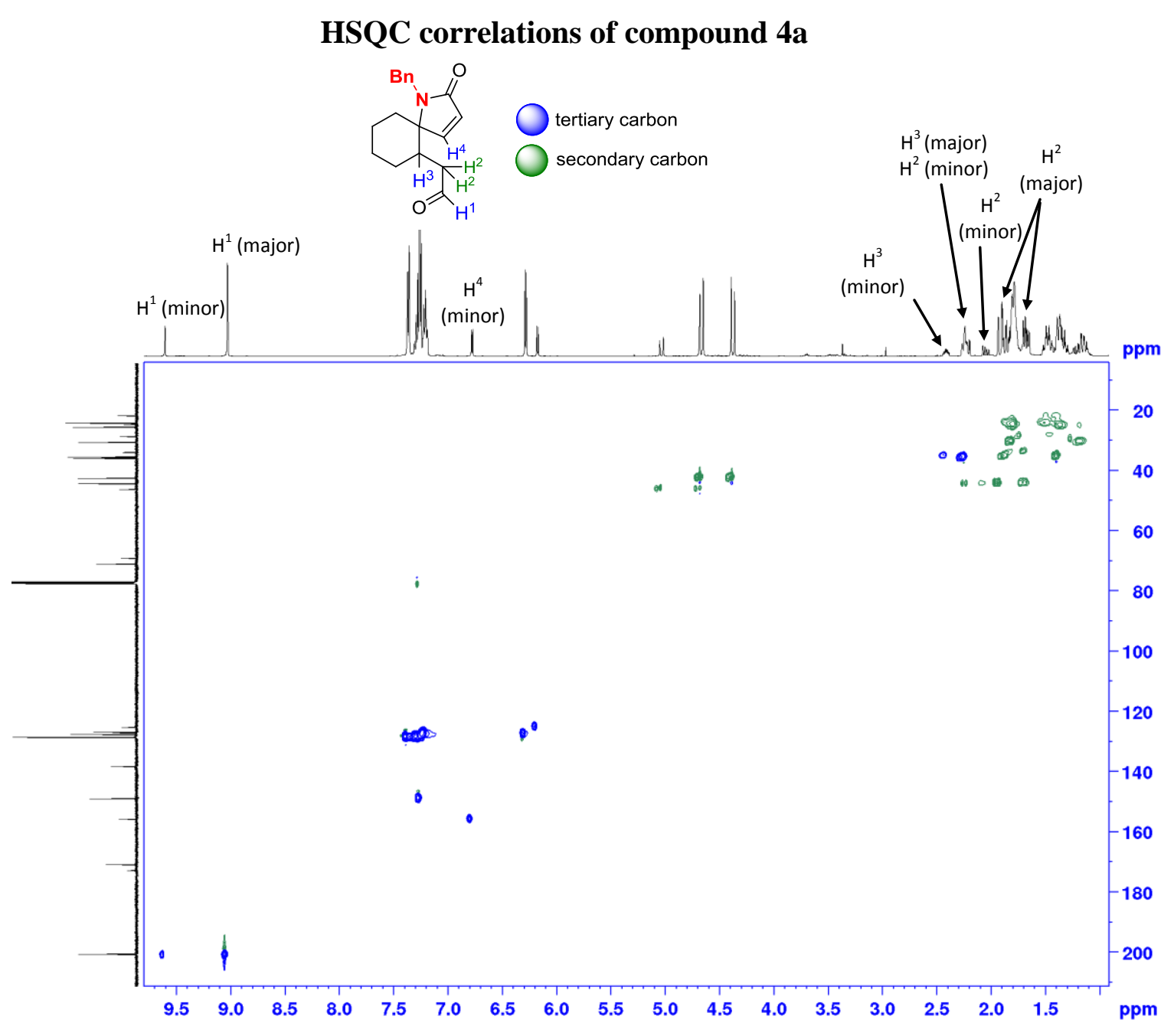


HMBC correlations of compound $4 a$

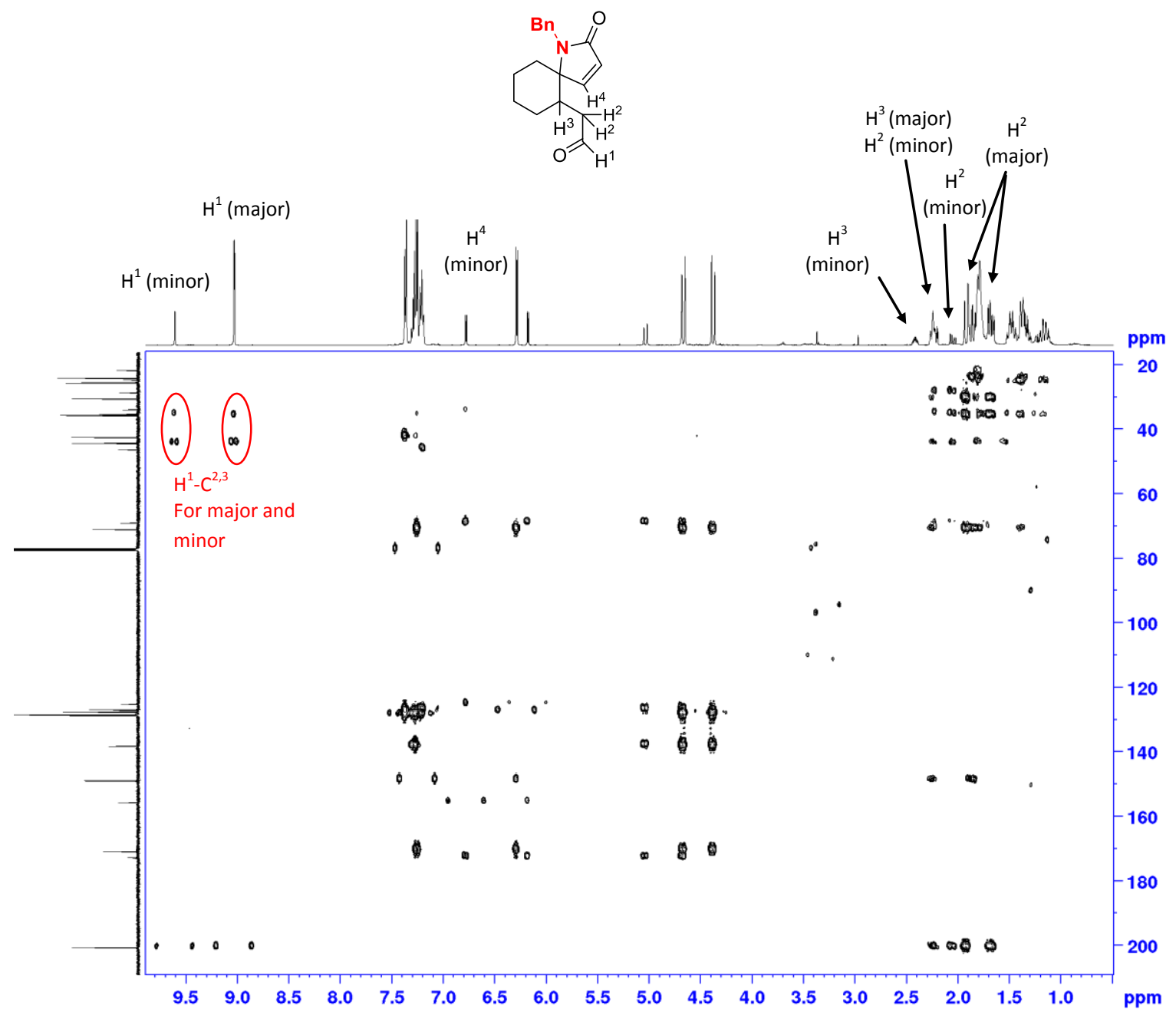




\section{Representative NOEs of compound 4a}

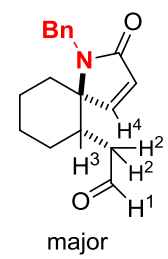<smiles>[H][Z]([H])([H])[C@H]1CCCC[C@]12CCC(=O)N2Cc1ccccc1</smiles>
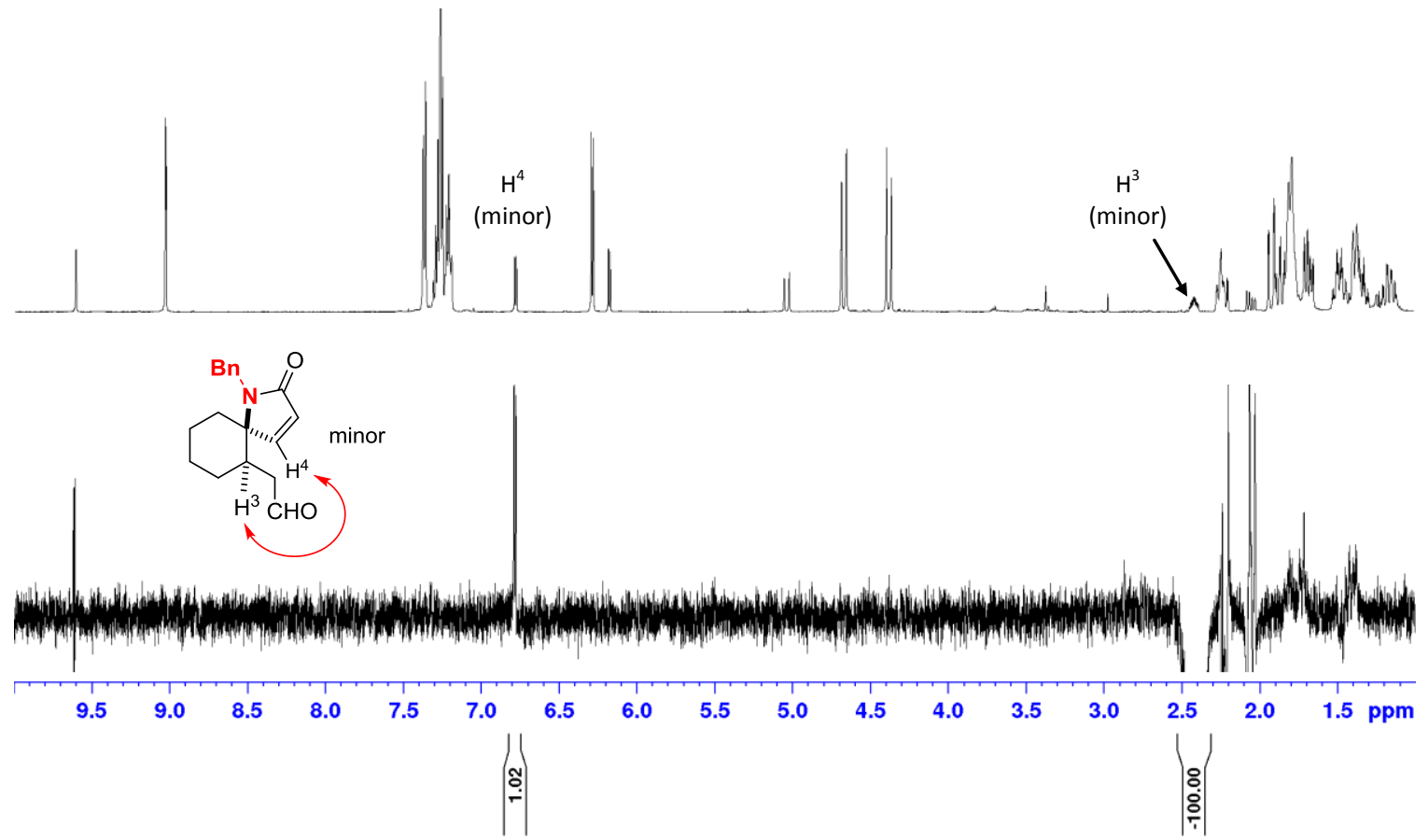


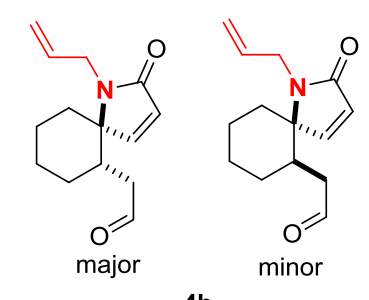

$\left(500 \mathrm{MHz}, \mathrm{CDCl}_{3}\right)$

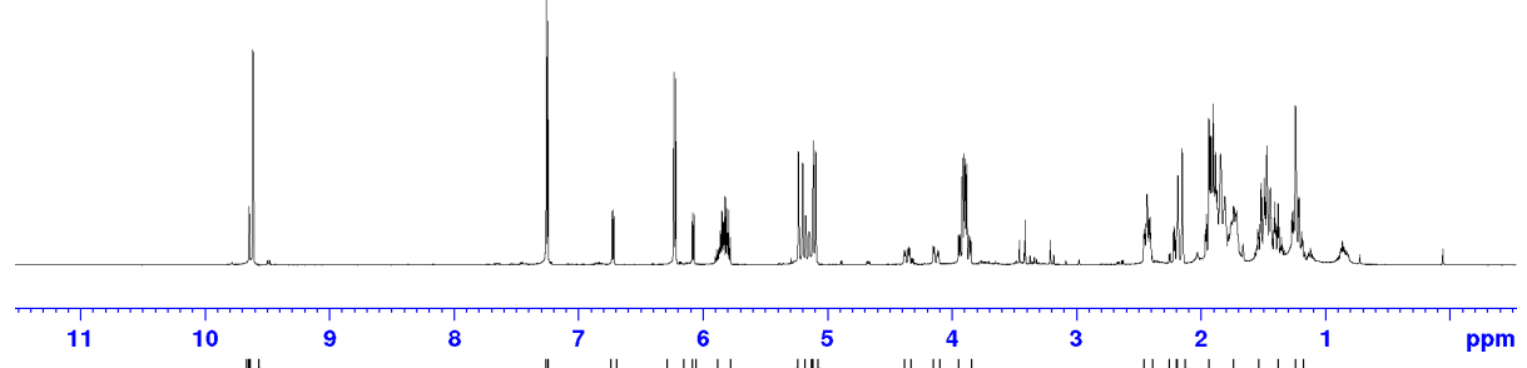

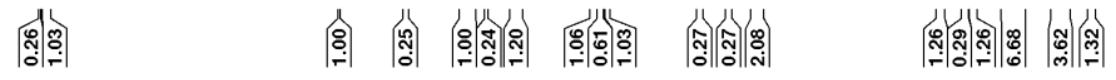

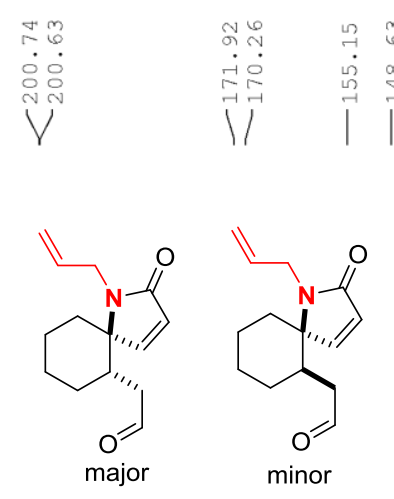

4b

(125 MHz, $\mathrm{CDCl}_{3}$ )

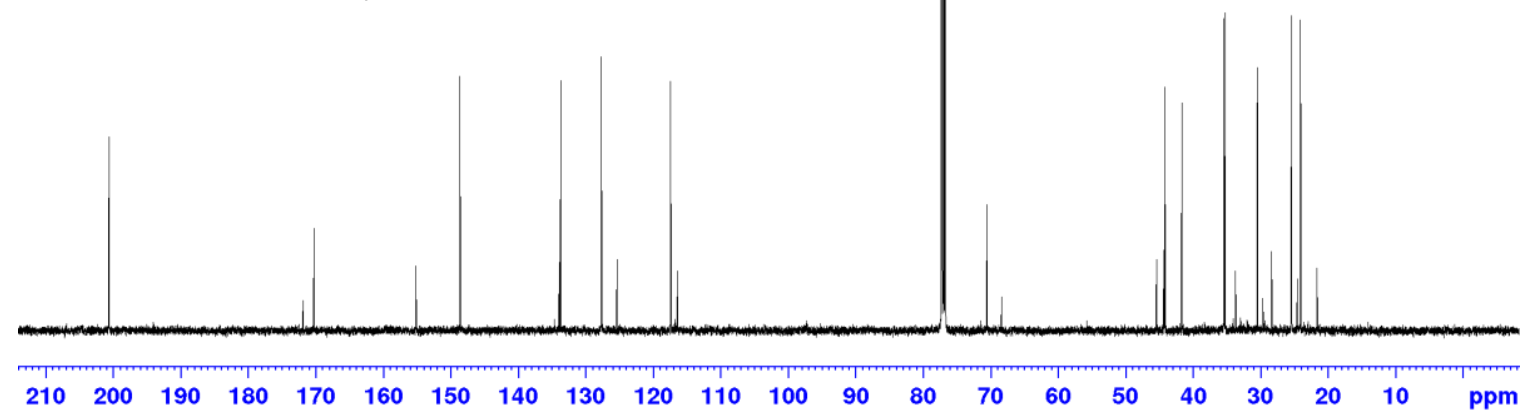



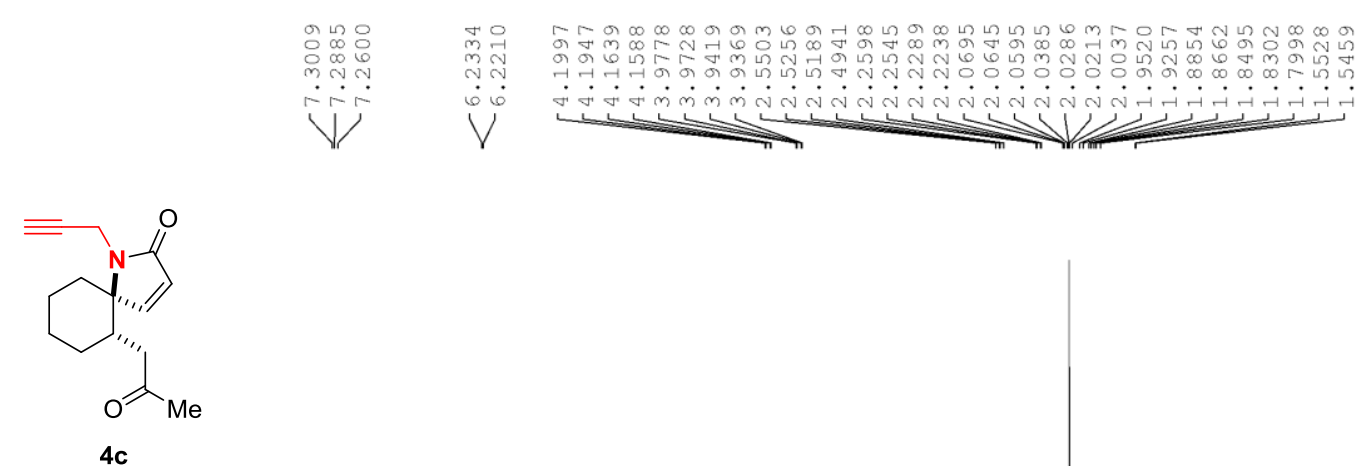

$\left(500 \mathrm{MHz}, \mathrm{CDCl}_{3}\right.$ )

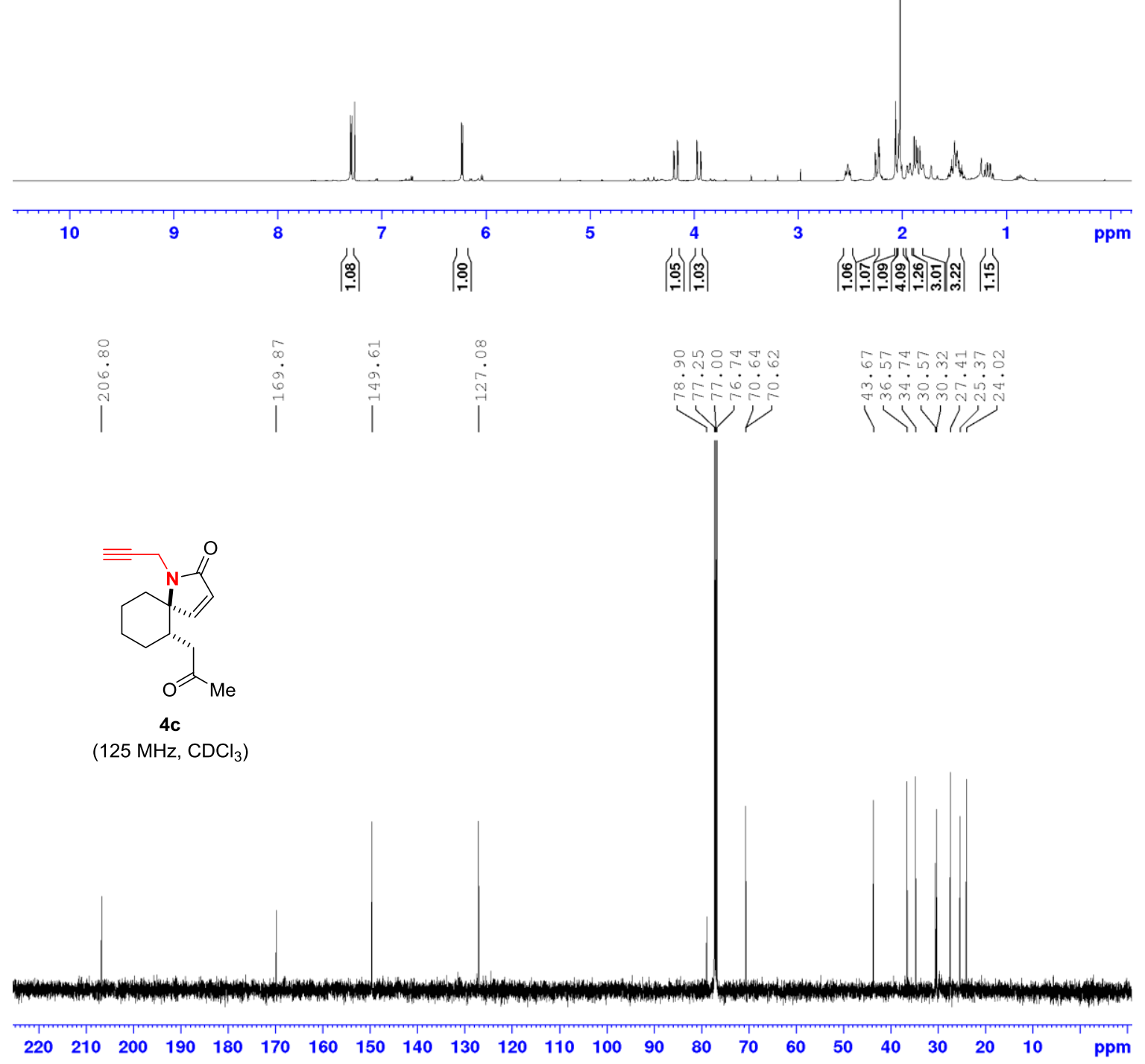


HMBC correlations of compound $4 \mathrm{c}$

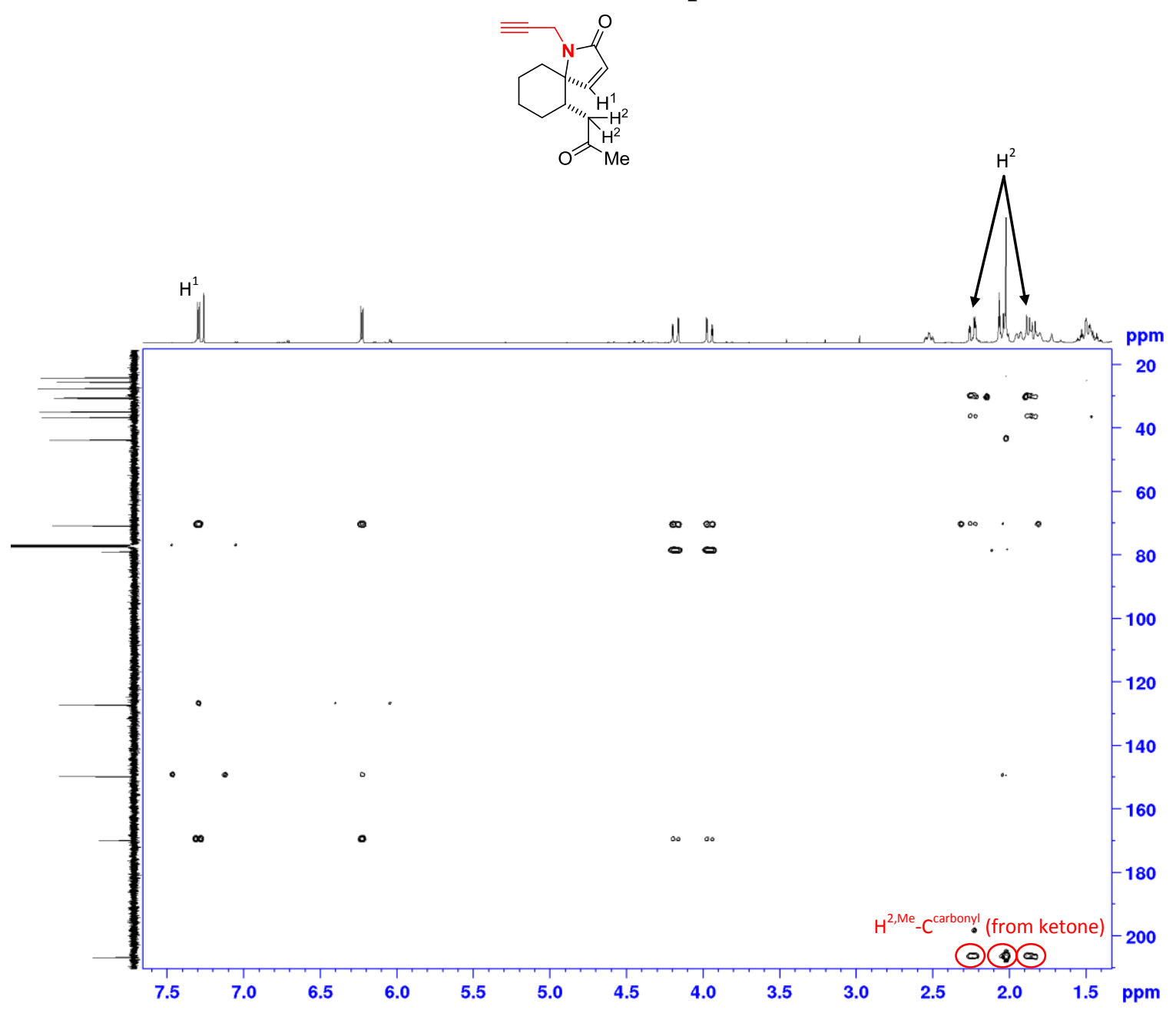




\section{Representative NOEs of compound $4 \mathrm{c}$}

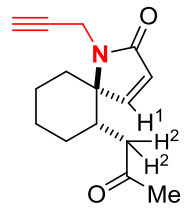

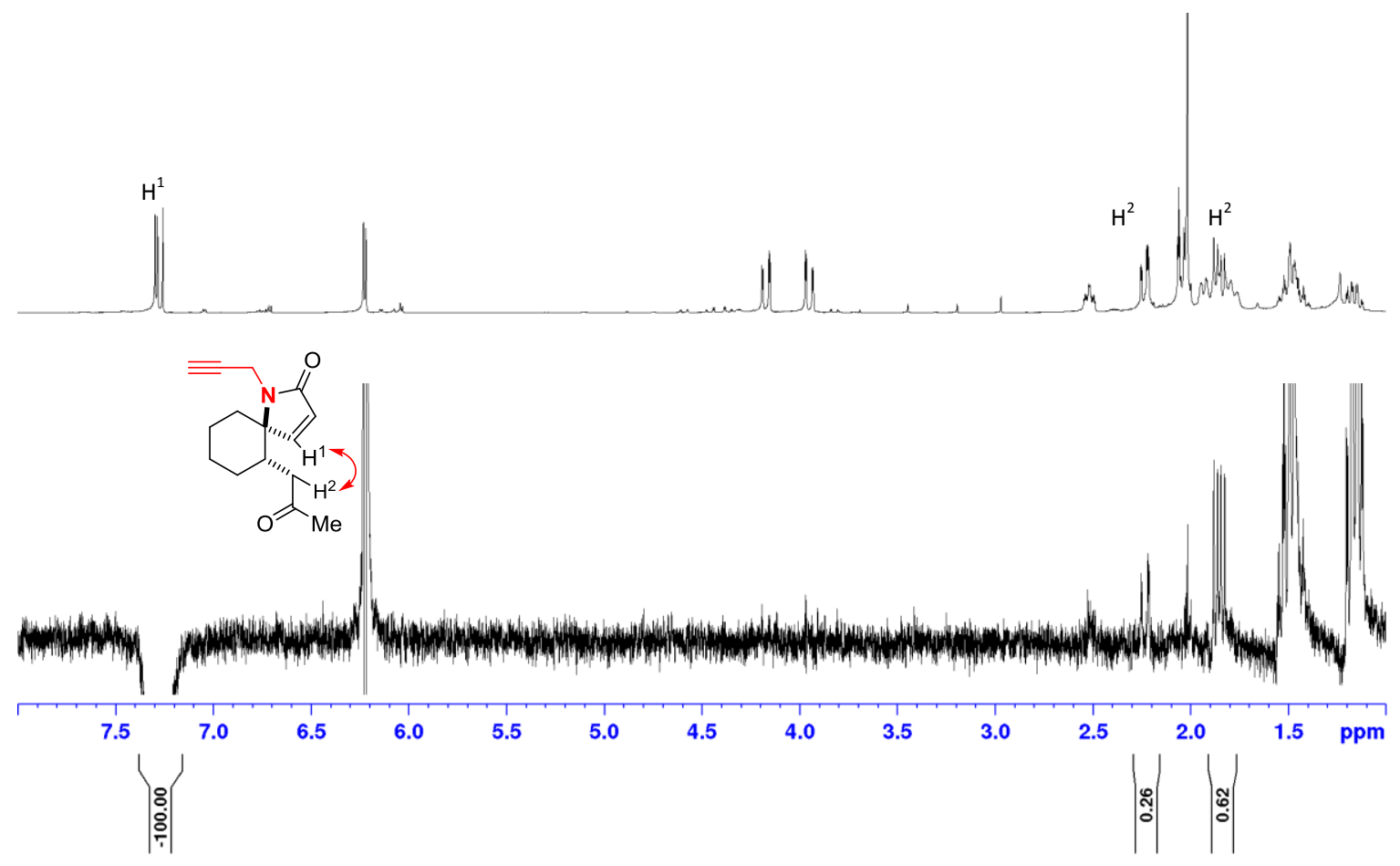




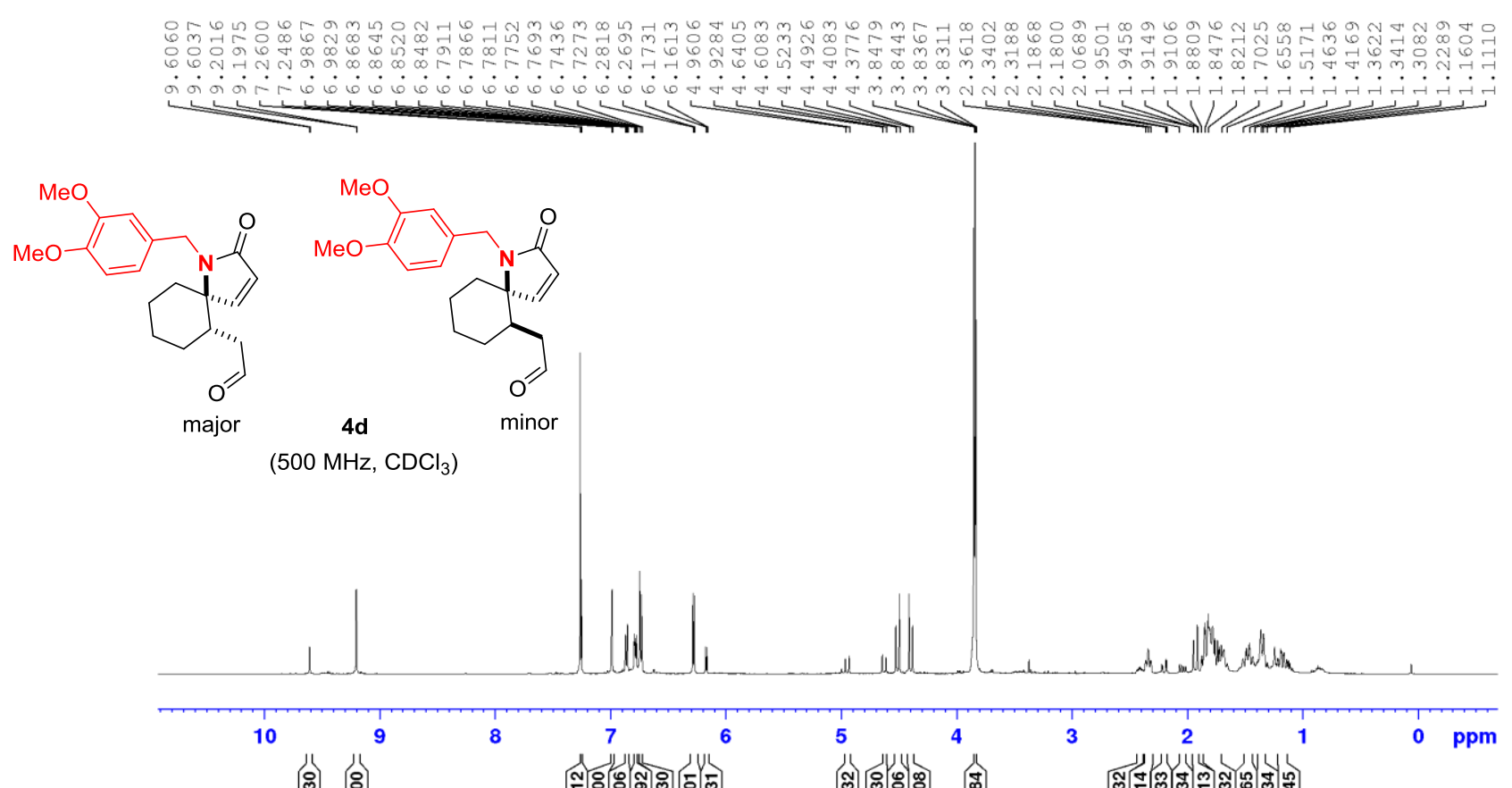

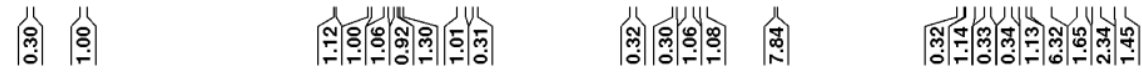
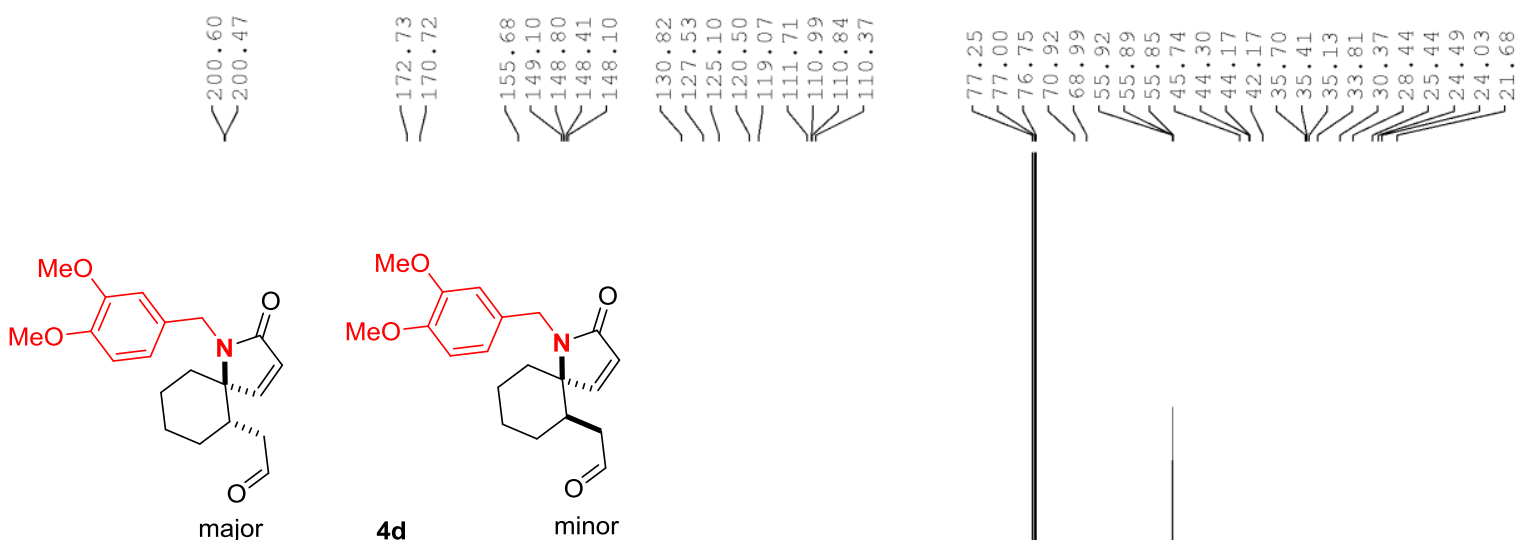

major

4d

$\left(125 \mathrm{MHz} \mathrm{CDCl}_{3}\right)$

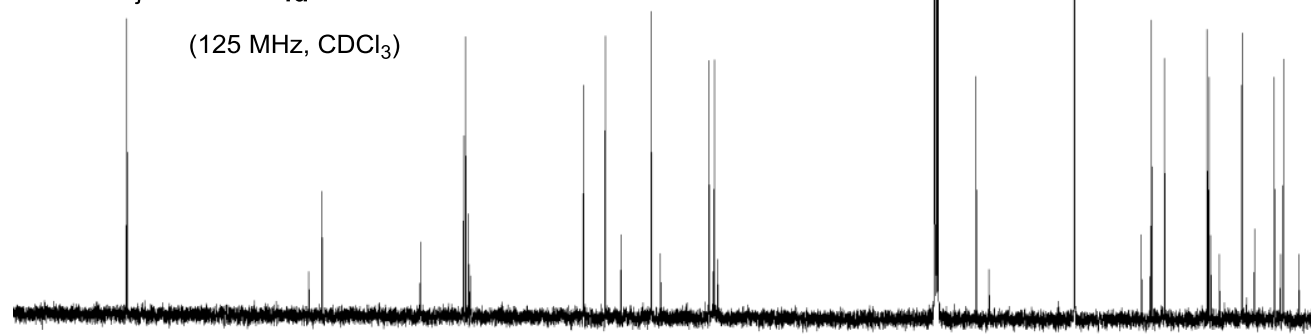

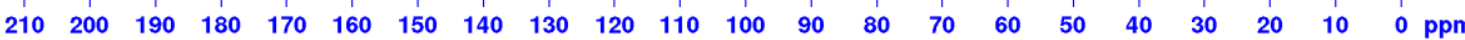



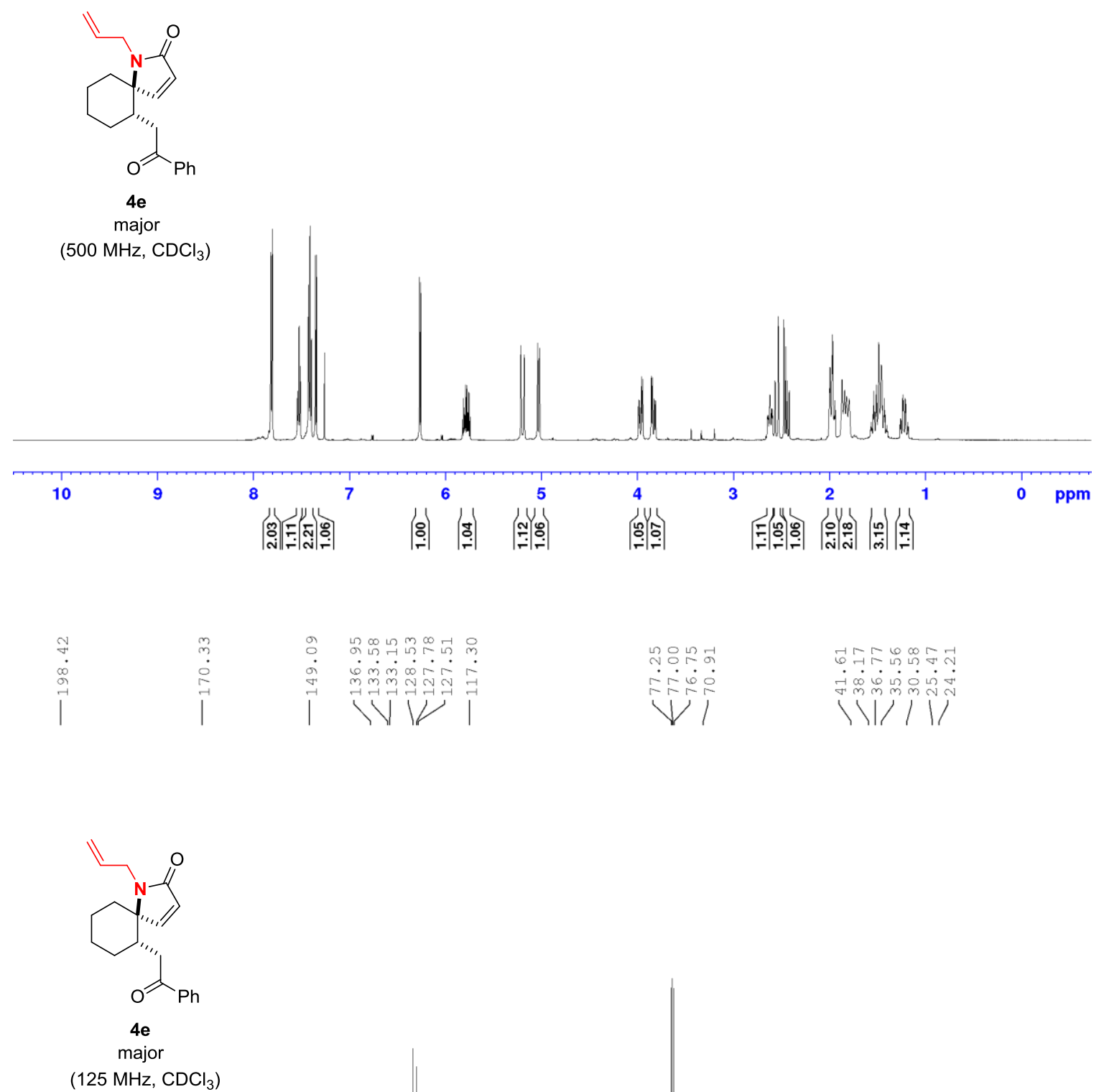

(125 MHz, $\mathrm{CDCl}_{3}$ )

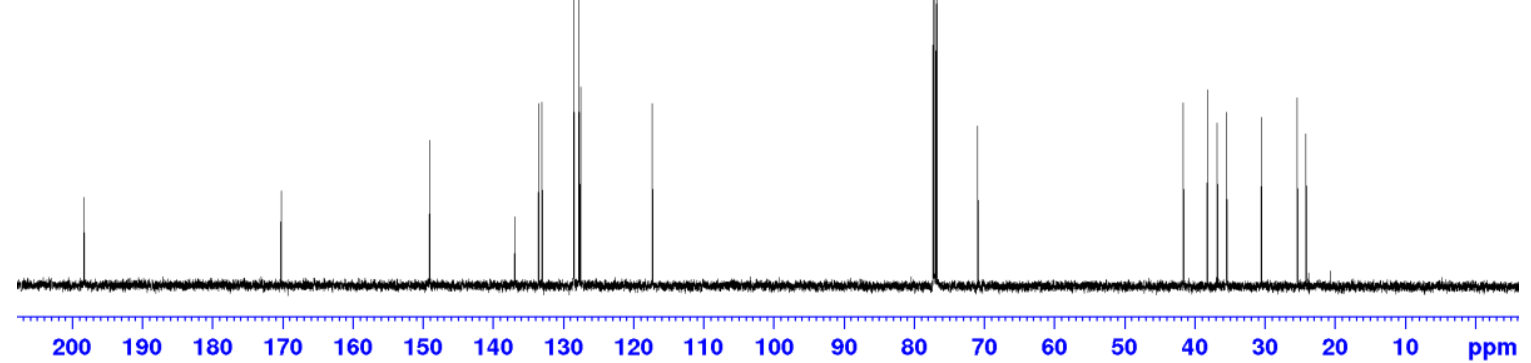


HSQC correlations of major isomer of compound $4 \mathrm{e}$

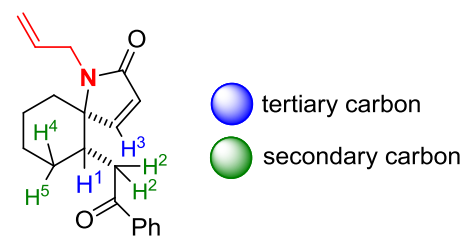

$4 \mathrm{e}$

major

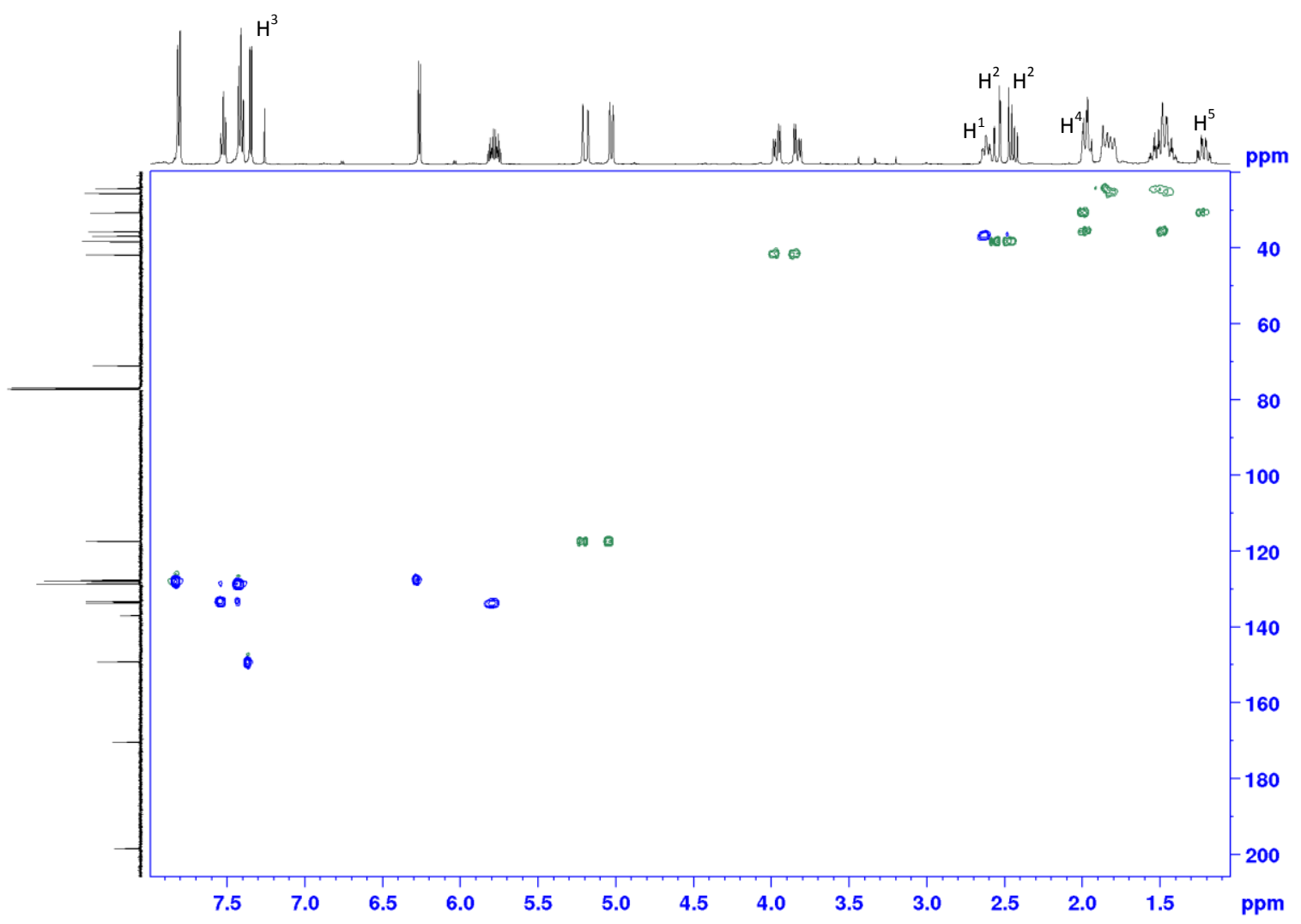




\section{HMBC correlations of major isomer of compound $4 \mathrm{e}$}
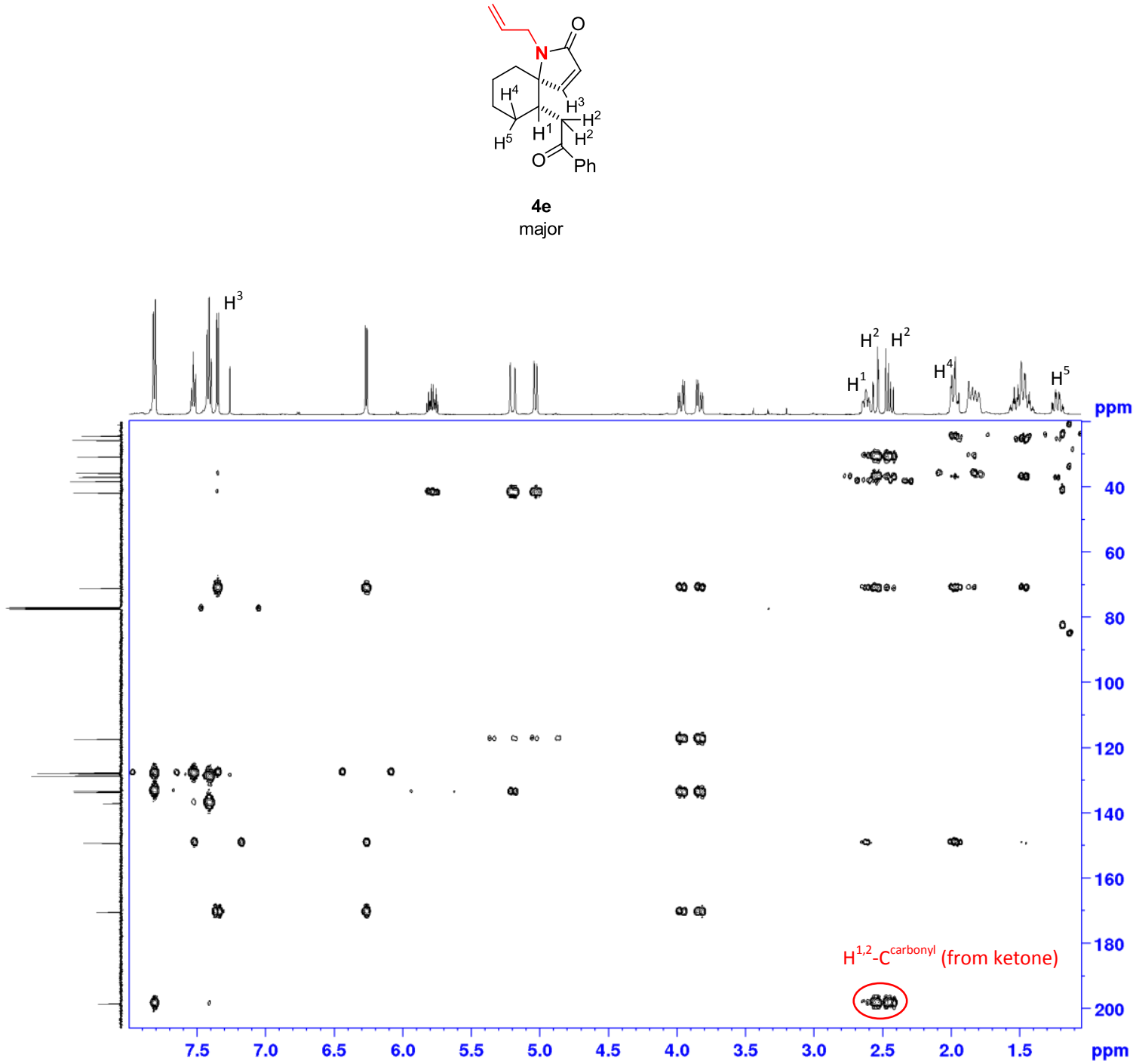
Representative NOEs of the major isomer of compound $4 \mathrm{e}$
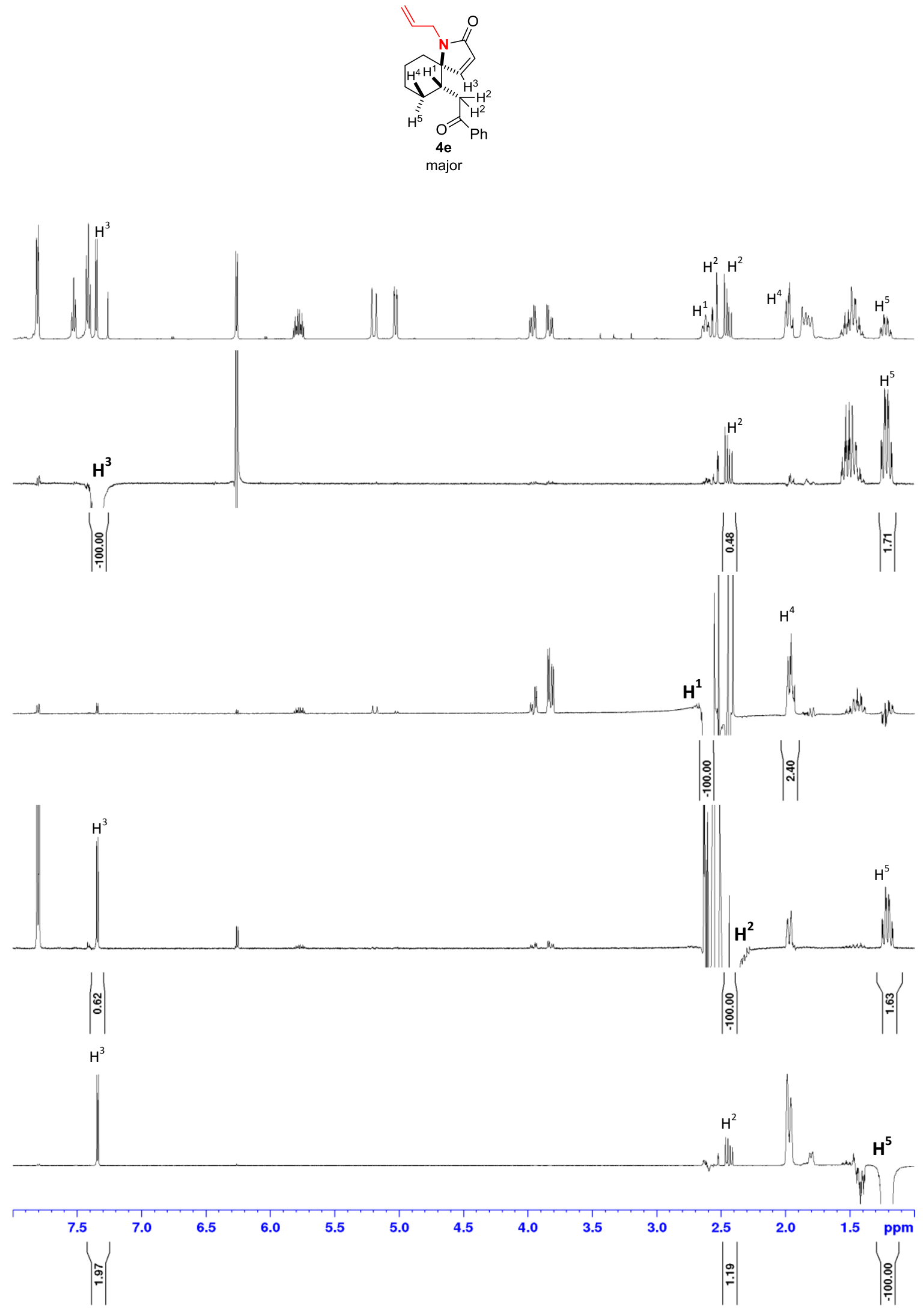

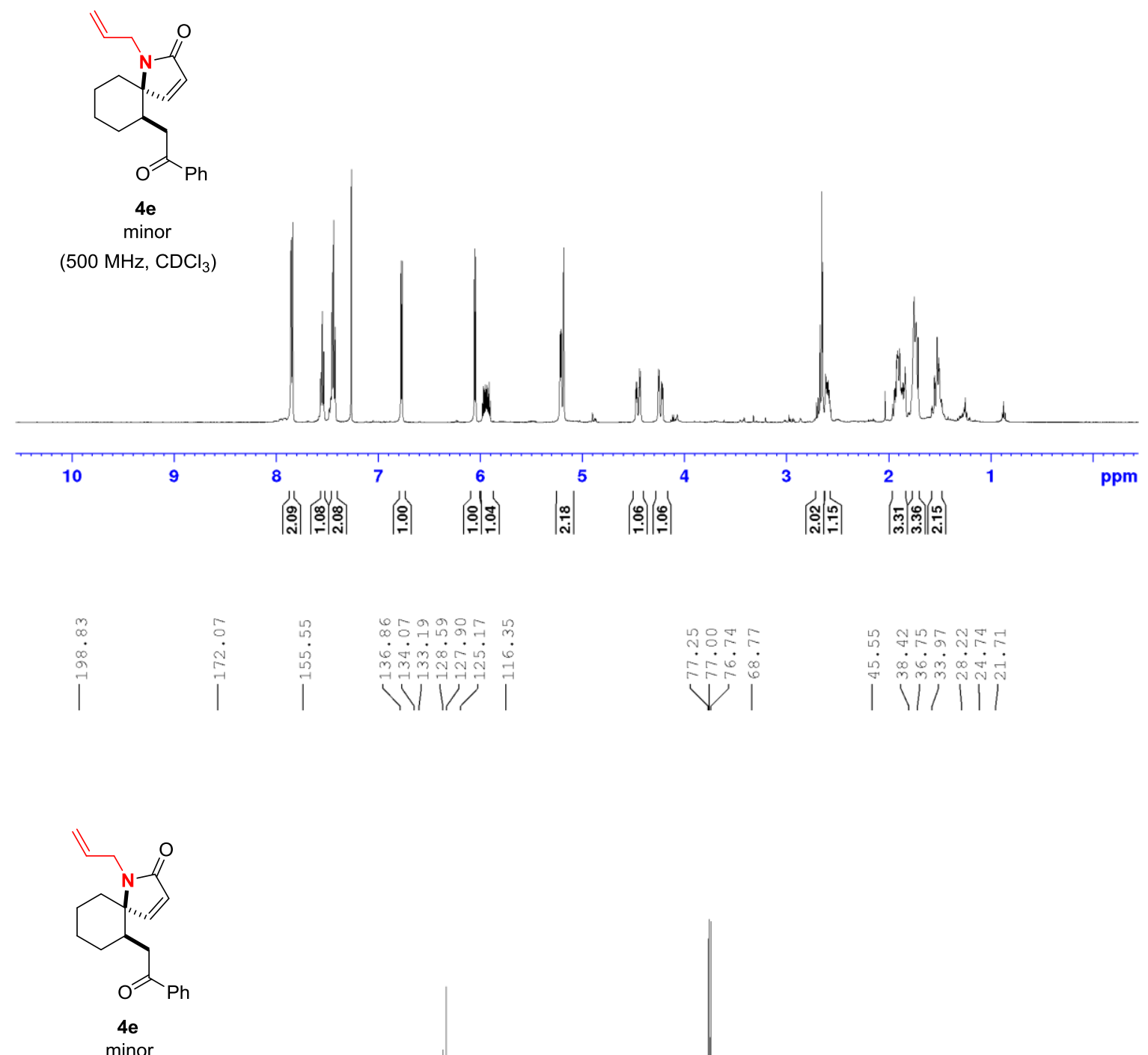

(125 MHz, $\mathrm{CDCl}_{3}$ )

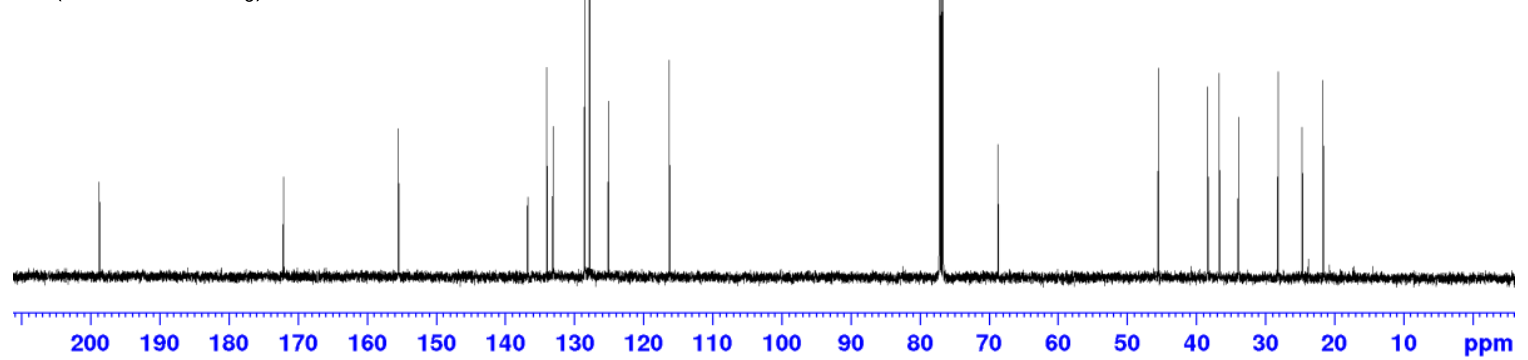




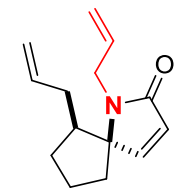

5

(500 $\mathrm{MHz}, \mathrm{CDCl}_{3}$ )
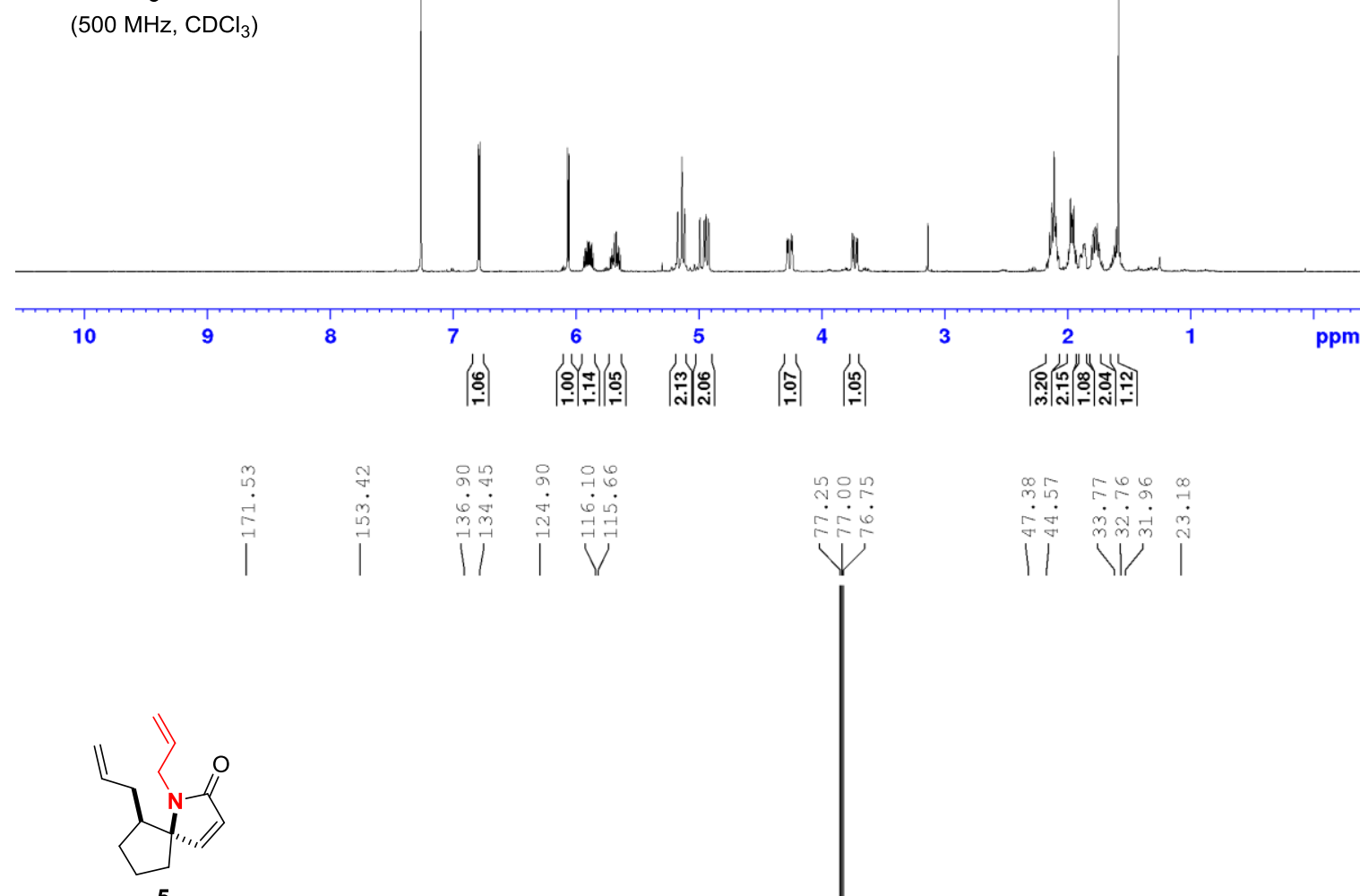

(125 $\mathrm{MHz}, \mathrm{CDCl}_{3}$ ) 


\section{HMBC correlations of compound 5}

The chemical shift of the $\alpha$-quaternary carbon next to the $\mathrm{N}$-atom was revealed by HMBC.
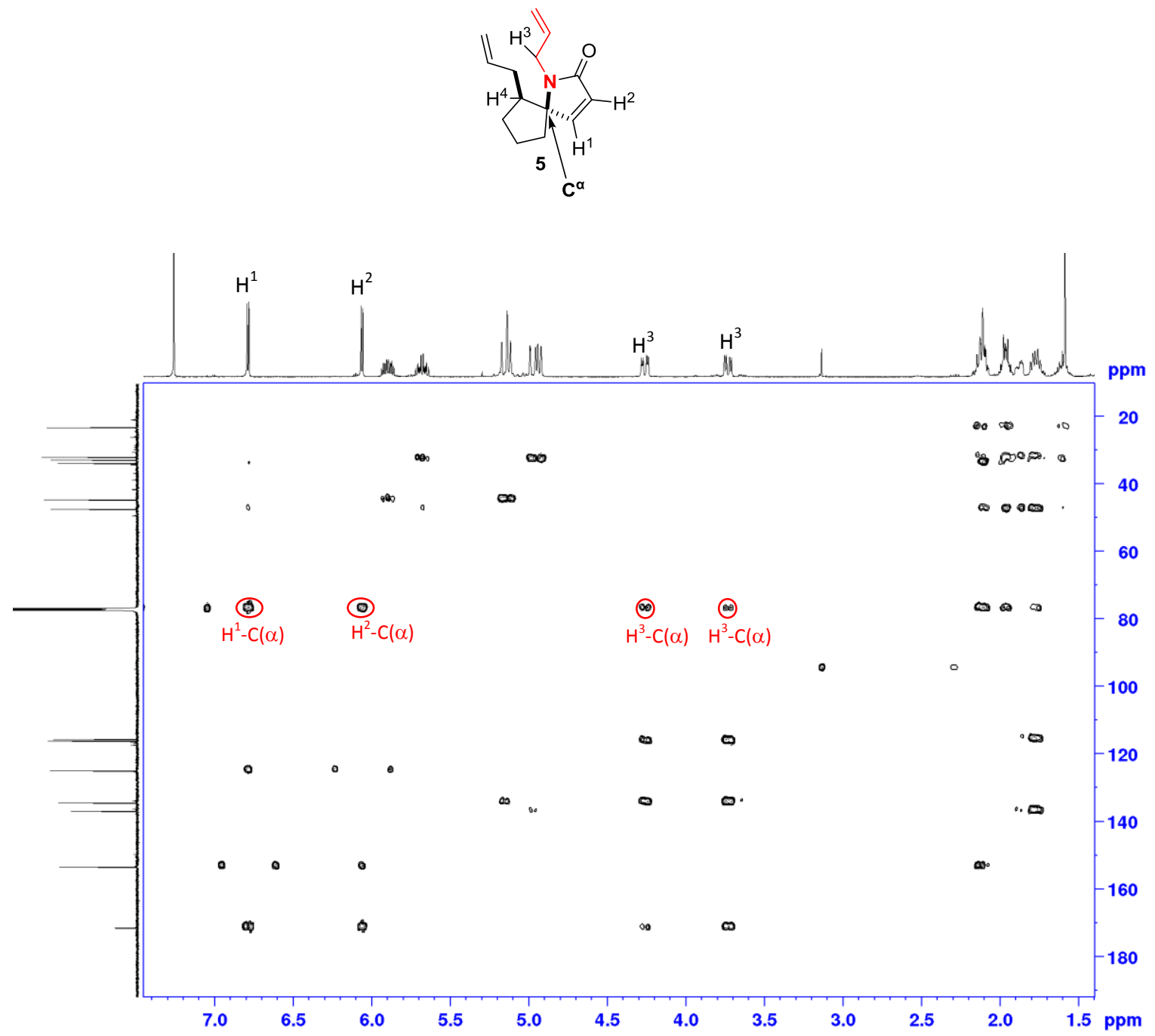


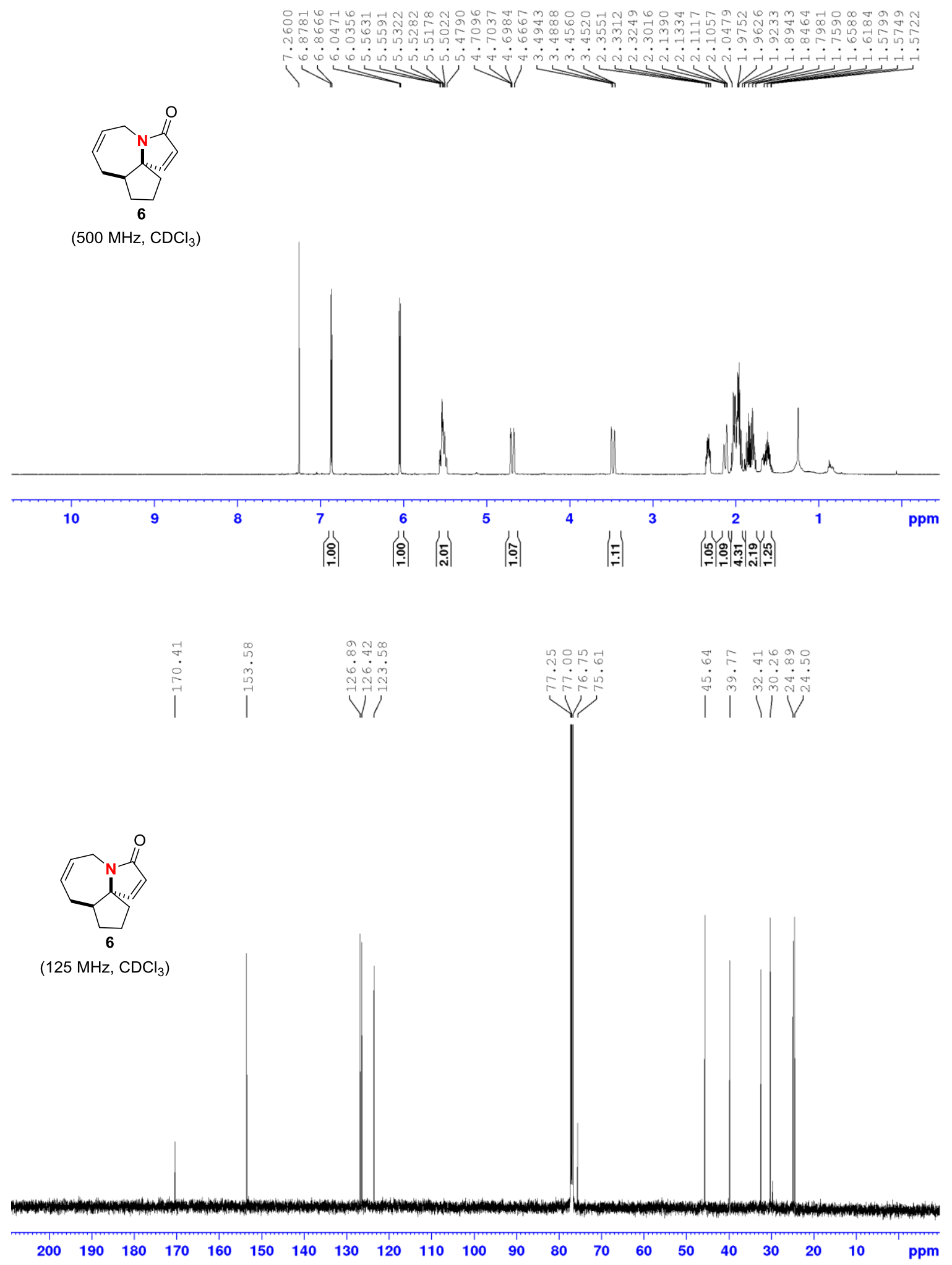




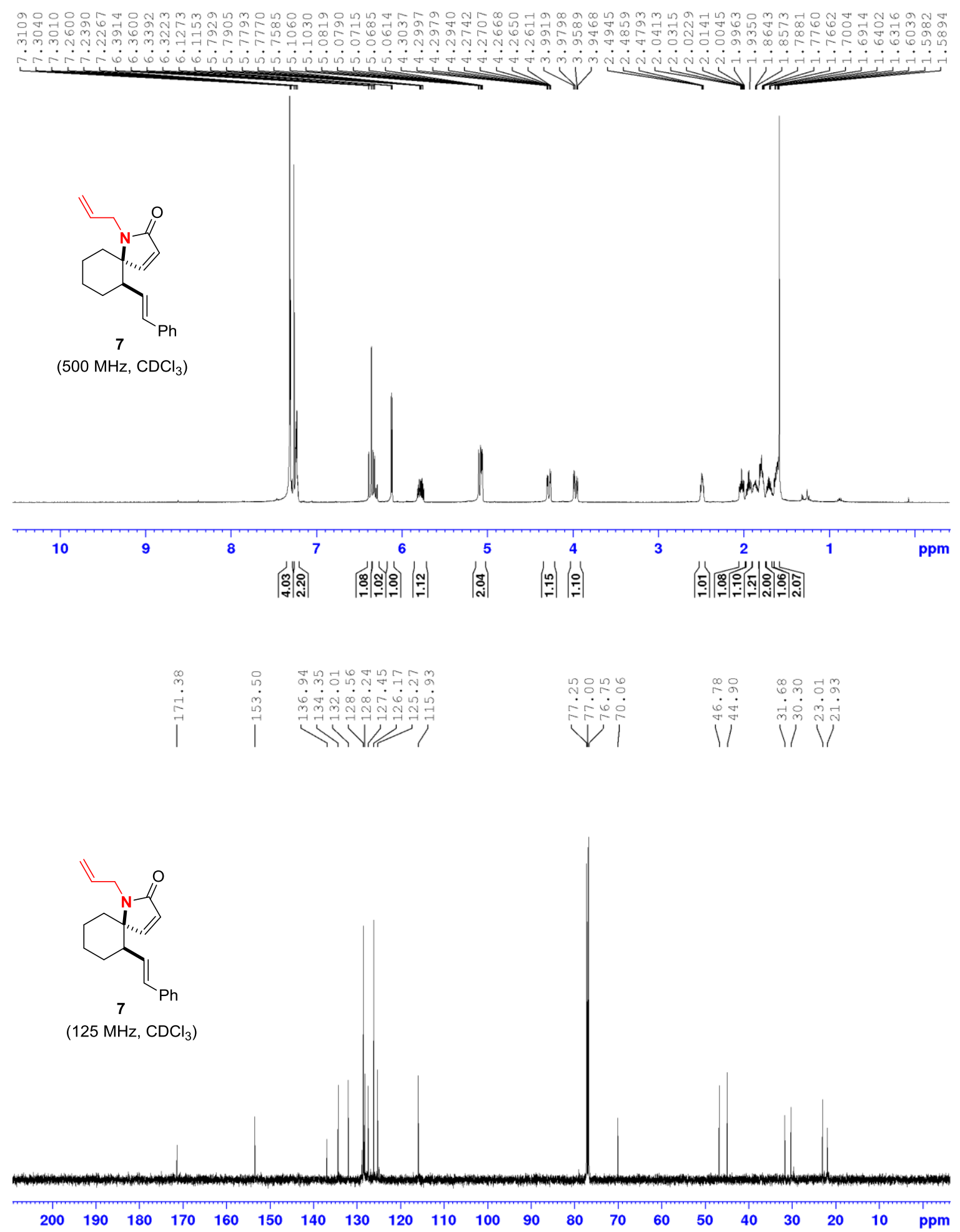




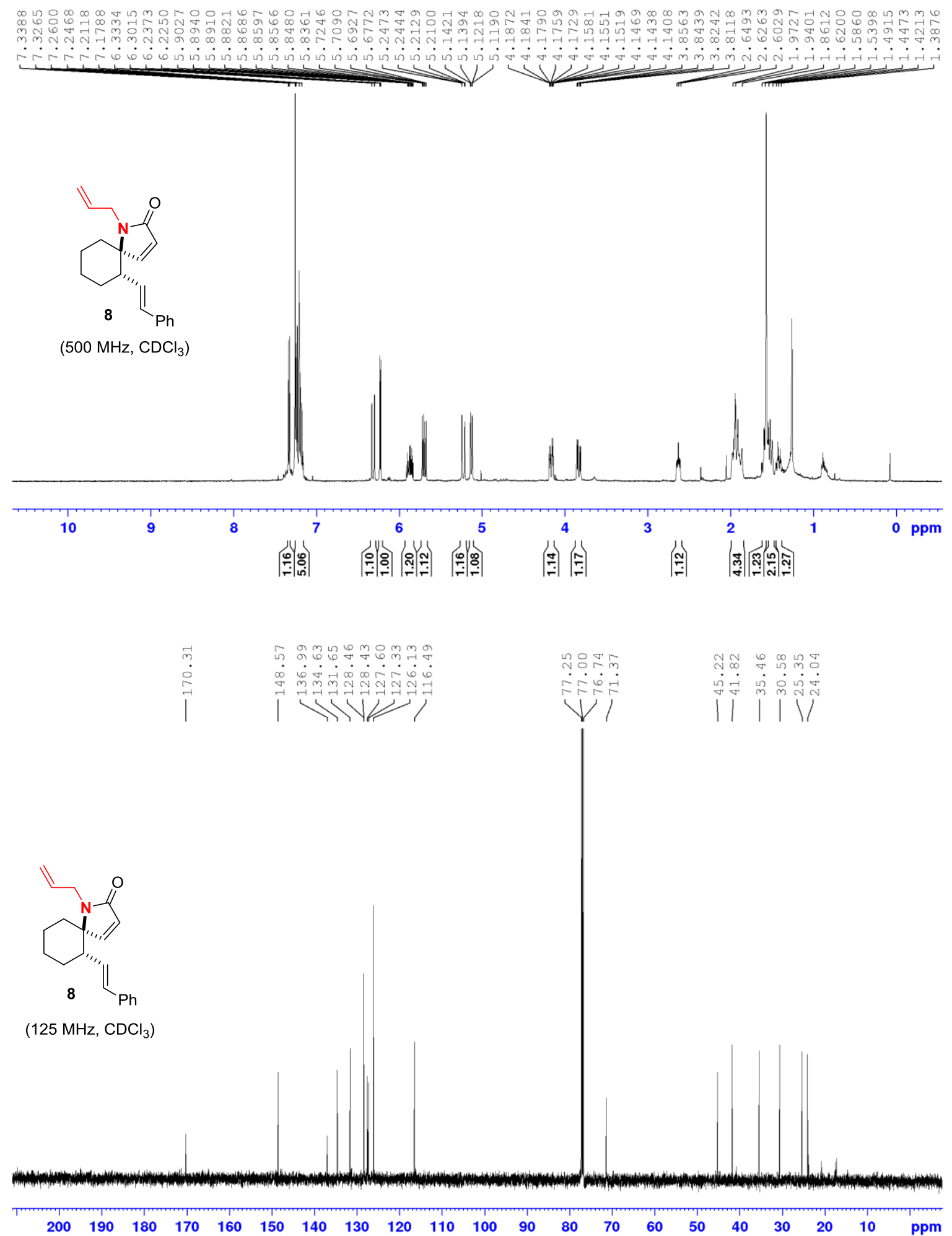




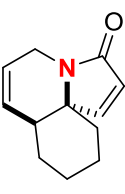

9

(500 MHz, $\mathrm{CDCl}_{3}$ )
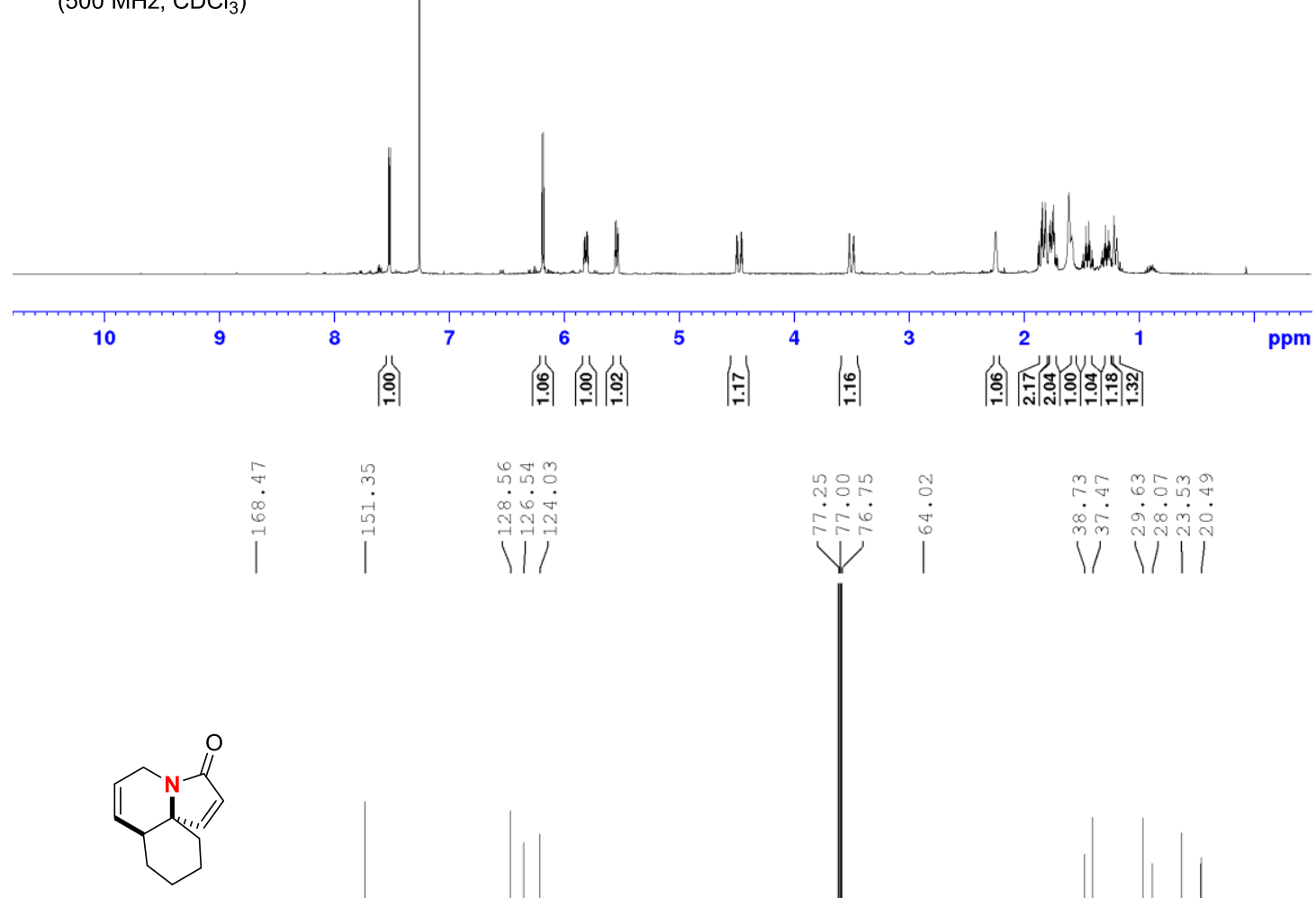

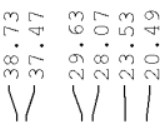

9

(125 MHz, $\mathrm{CDCl}_{3}$ )

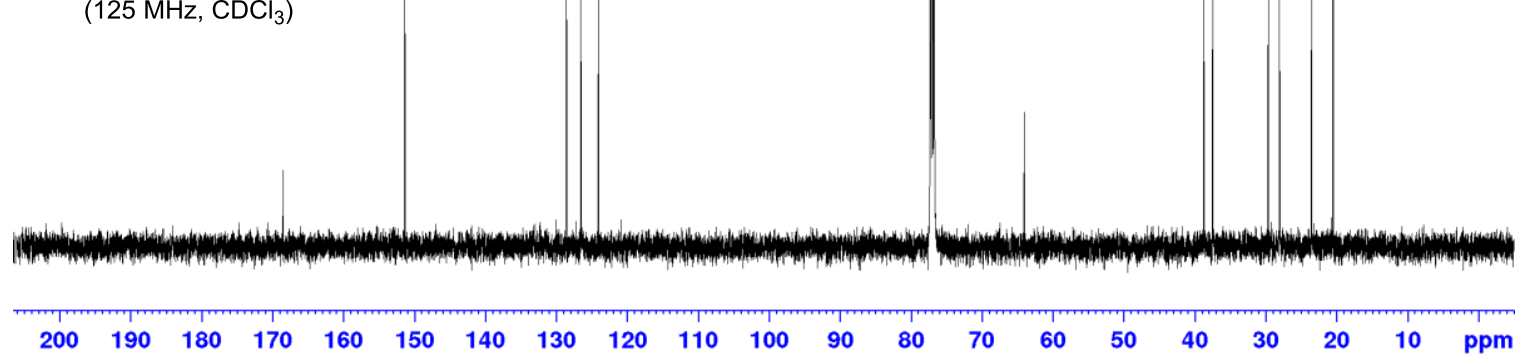




\section{HSQC correlations of compound 9}
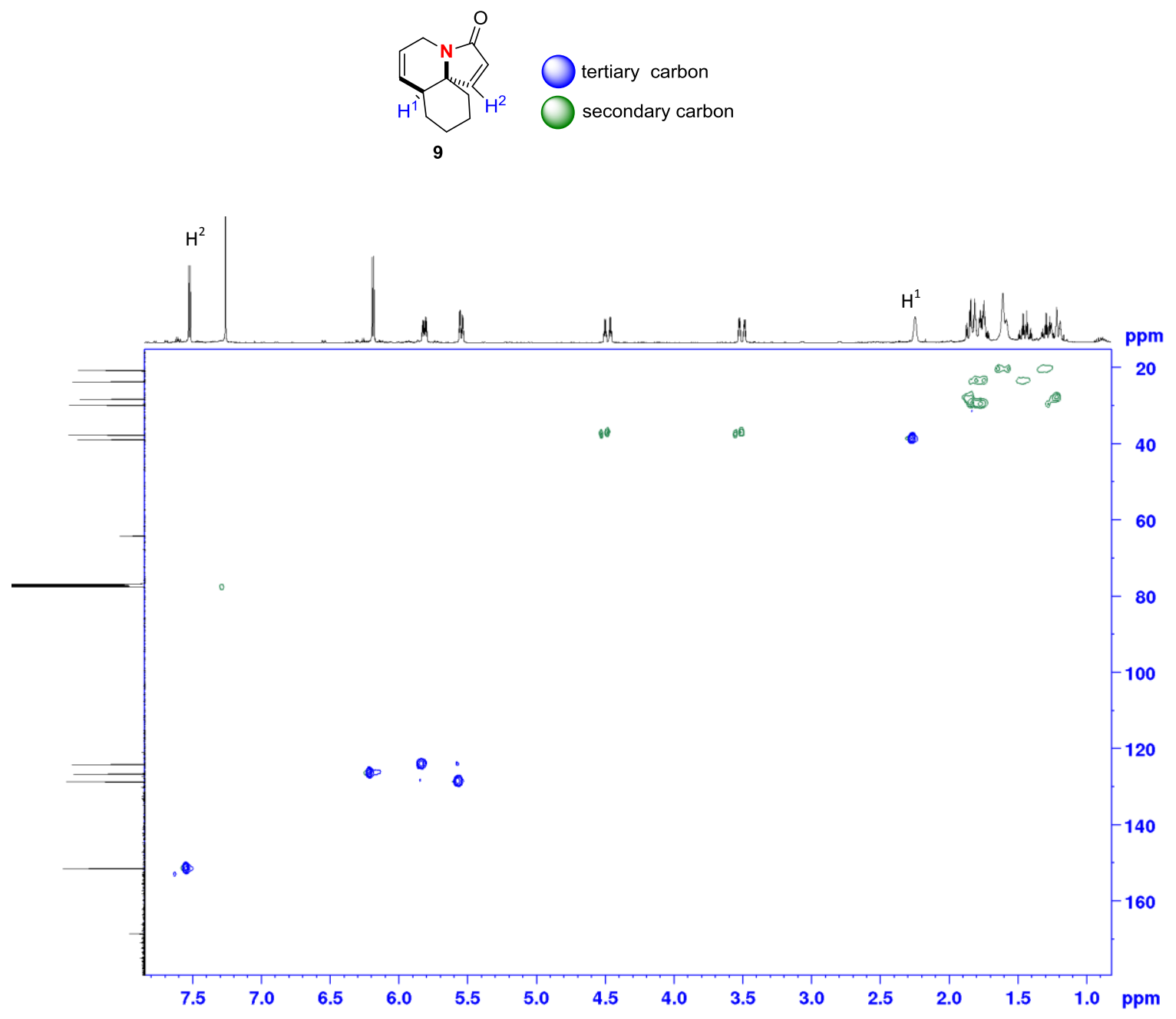
Representative NOEs of compound 9<smiles>O=C1C=CC2CCCC3C=CCN1C32</smiles>

9

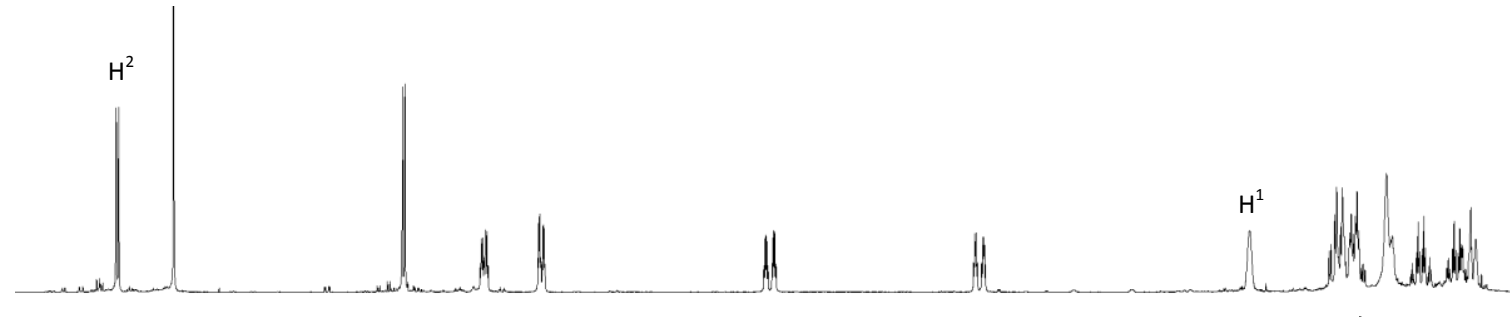

$\mathrm{H}^{2}$

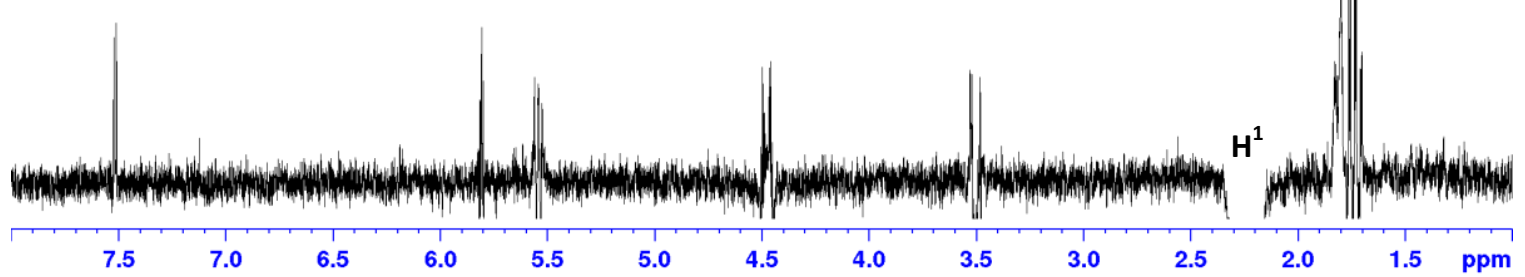
क्.

: 


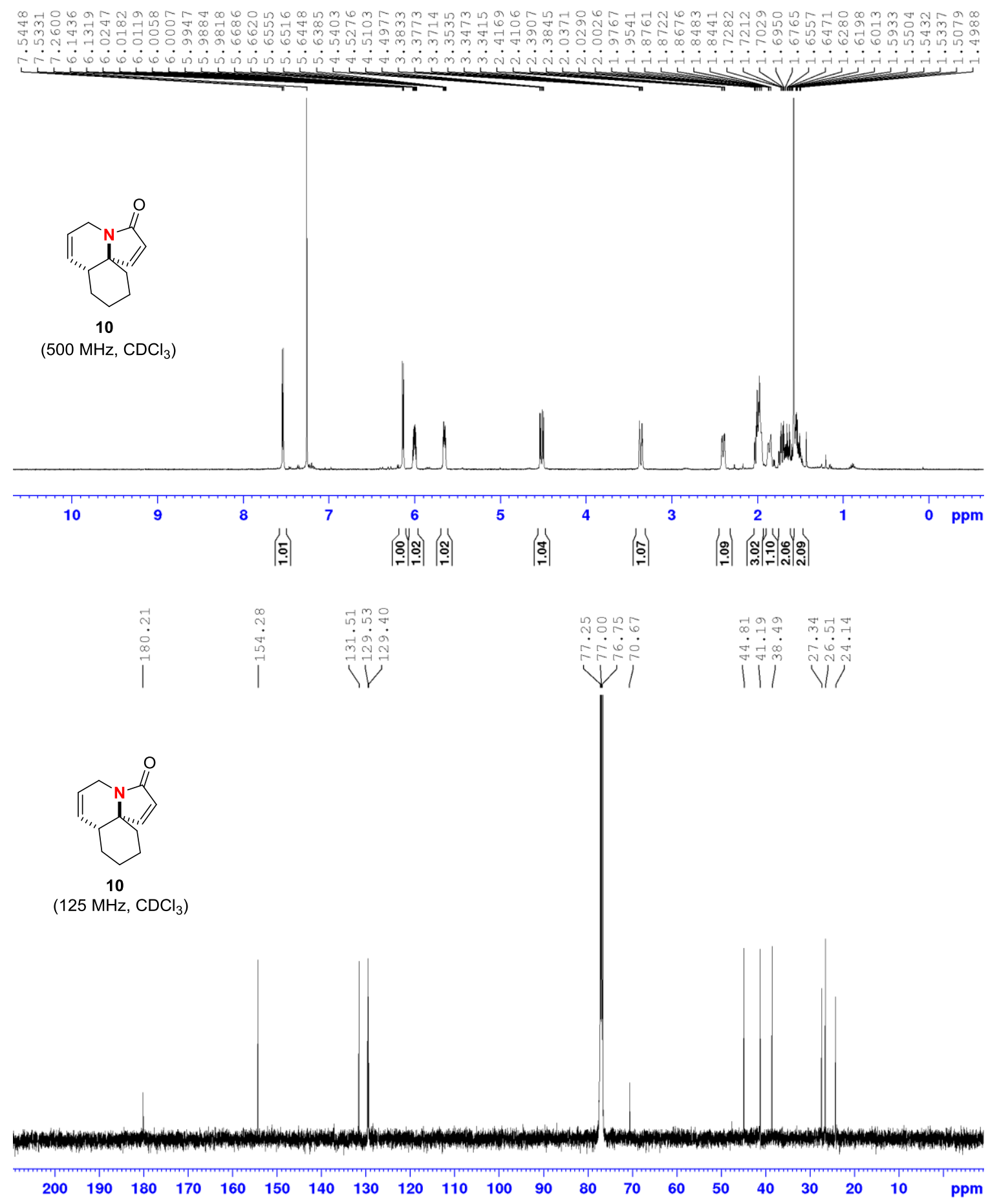

